\title{
Package Equivalent Reactor Networks as Reduced Order Models for Use with CAPE- OPEN Compliant Simulations
}

\section{Final Report}

Report Period Start Date: October 1, 2009

Report Period End Date: March 31, 2013

Principal Authors: E. Meeks, C.-P. Chou, and T. Garratt

Recipient: Reaction Design

Date Report was Issued: March 2013

DOE Award Number: DE-FC26-FE0001074

Submitting Organization: Reaction Design 5930 Cornerstone Court West, Suite 230 San Diego, CA 92121

Report Number: DOE/FE0001074-4 


\section{Disclaimer}

This report was prepared as an account of work sponsored by an agency of the United States Government. Neither the United States Government nor any agency thereof, nor any of their employees, makes any warranty, express or implied, or assumes any legal liability or responsibility for the accuracy, completeness, or usefulness of any information, apparatus, product, or process disclosed, or represents that its use would not infringe privately owned rights. Reference herein to any specific commercial product, process, or service by trade name, trademark, manufacturer, or otherwise does not necessarily constitute or imply its endorsement, recommendation, or favoring by the United States Government or any agency thereof. The views and opinions of authors expressed herein do not necessarily state or reflect those of the United States Government or any agency thereof. 


\section{ABSTRACT}

Engineering simulations of coal gasifiers are typically performed using computational fluid dynamics (CFD) software, where a 3-D representation of the gasifier equipment is used to model the fluid flow in the gasifier and source terms from the coal gasification process are captured using discrete-phase model source terms. Simulations using this approach can be very time consuming, making it difficult to imbed such models into overall system simulations for plant design and optimization. For such system-level designs, process flowsheet software is typically used, such as Aspen Plus ${ }^{\circledR}$ [1], where each component where each component is modeled using a reduced-order model. For advanced power-generation systems, such as integrated gasifier/gas-turbine combined-cycle systems (IGCC), the critical components determining overall process efficiency and emissions are usually the gasifier and combustor. Providing more accurate and more computationally efficient reduced-order models for these components, then, enables much more effective plant-level design optimization and design for control.

Based on the CHEMKIN-PRO and ENERGICO software, we have developed an automated methodology for generating an advanced form of reduced-order model for gasifiers and combustors. The reducedorder model offers representation of key unit operations in flowsheet simulations, while allowing simulation that is fast enough to be used in iterative flowsheet calculations. Using high-fidelity fluiddynamics models as input, Reaction Design's ENERGICO® [2] software can automatically extract equivalent reactor networks (ERNs) from a CFD solution. For the advanced reduced-order concept, we introduce into the ERN a much more detailed kinetics model than can be included practically in the CFD simulation. The state-of-the-art chemistry solver technology within CHEMKIN-PRO allows that to be accomplished while still maintaining a very fast model turn-around time. In this way, the ERN becomes the basis for high-fidelity kinetics simulation, while maintaining the spatial information derived from the geometrically faithful CFD model.

The reduced-order models are generated in such a way that they can be easily imported into a process flowsheet simulator, using the CAPE-OPEN architecture for unit operations. The ENERGICO/CHEMKIN-PRO software produces an ERN-definition file that is read by a dynamically linked library (DLL) that can be easily linked to any CAPE-OPEN compliant software. The plug-in unitoperation module has been successfully demonstrated for complex ERNs of coal gasifiers, using both Aspen Plus and COFE process flowsheet simulators through this published CAPE-OPEN interface. 


\section{Table of Contents}



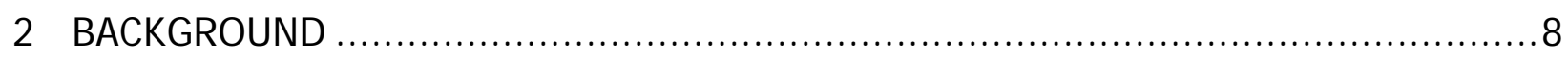

2.1 Reactor Networks and ENERGICO $®$ Software ..................................................... 8

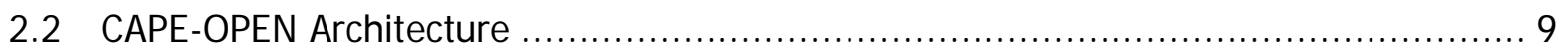

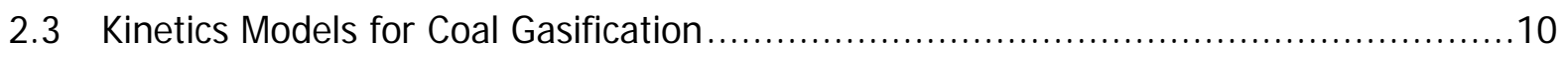

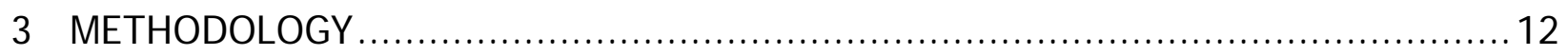

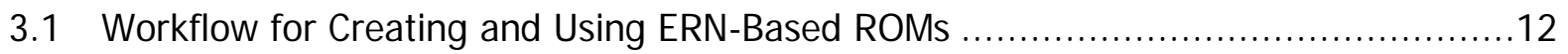

3.1.1 Exporting the CFD Solution for Use in ENERGICO................................................ 12

3.1.2 Representing Coal Volatiles in the ERN's Detailed Chemistry ...................................... 14

3.1.3 Performing Zone Partitioning within ENERGICO to Define the ERN …….................... 17

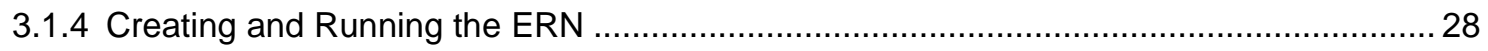

3.1.5 Validating the ERN through Comparison to the CFD ............................................... 29

3.1.6 Exporting the ERN Model for Use with the CAPE-OPEN Interface .............................. 30

3.1.7 Running the CHEMKIN-CO ERN within Aspen Plus ..................................................30

3.2 Implementation of the CAPE-OPEN I nterface for ERN Models .................................31

3.2.1 Lumping Method Implementation to Assure Mass and Element Conservation ................. 32

3.3 Development of a New Coal Kinetics Module.................................................... 34

3.3.1 Surface Chemistry Representation of Devolatilization.................................................. 36

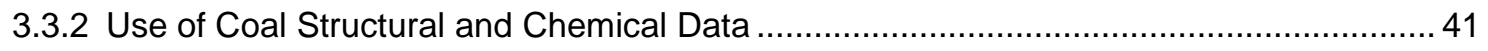

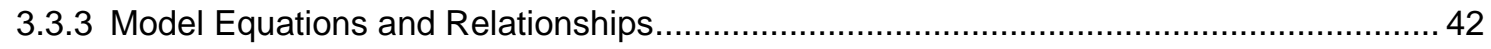

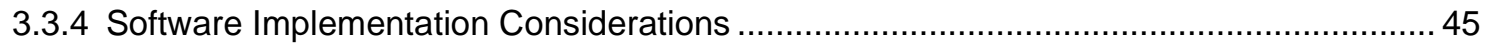

4 RESULTS AND DISCUSSION ............................................................ 48

4.1 Equivalent Reactor Network for NETL Single-Stage Gasifier ..........................48

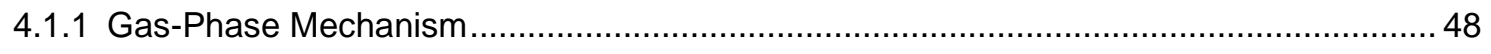

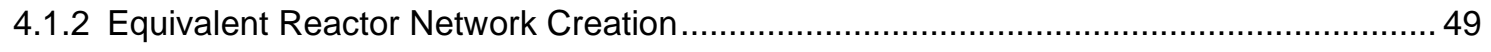

4.2 Comparisons between the ROM and the Original CFD Model...........................50

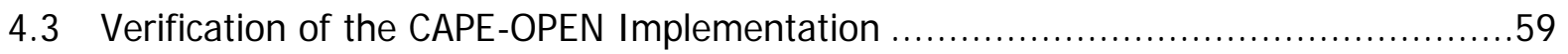

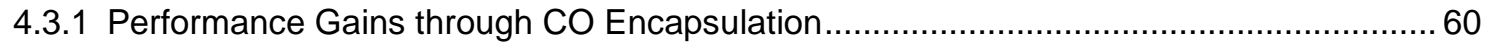

4.4 Chemical Percolation Devolatilization Model Testing ..................................60

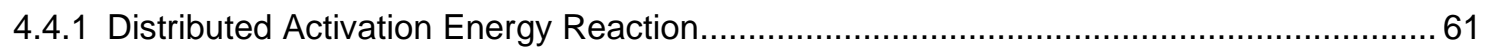

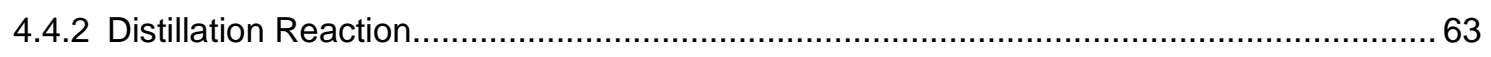

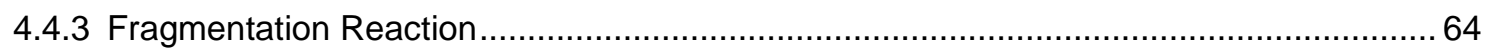




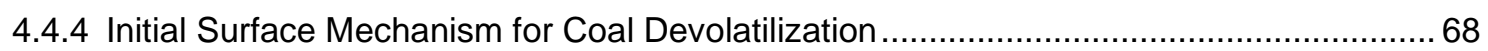

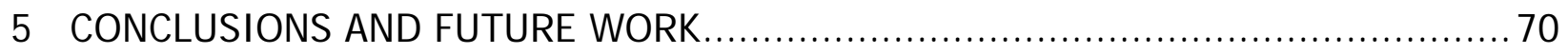



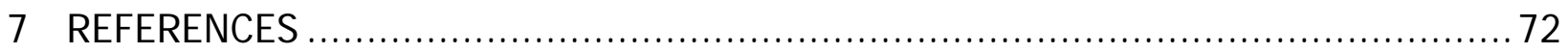




\section{Executive Summary}

The method currently in use for generating Reduced Order Models (ROMs) for the Advanced Process Engineering Co-Simulator (APECS) project at NETL involves fitting of a parameterized model to results from many CFD simulations [3]. This approach requires running the high-fidelity flow simulations many times in advance of creating the ROM; this process can take days or even weeks of compute time. In addition, the ROM that results does not provide any additional insight or capability compared to the original CFD simulations; it only provides an approximation of the original model that runs faster, but it cannot extrapolate outside of the conditions run in CFD.

In contrast, while an equivalent-reactor network can also be used as a ROM due to its fast run time, it additionally offers some significant advantages over the fitting approach. An ERN that consists of Perfectly-Stirred Reactor (PSR) models can be automatically generated for coal gasifiers from CFD simulation results. The equivalent reactor network (ERN) solves conservation equations for a geometrically coarse representation of the flow and then adds to the approximated flow model the supplemental information provided by a detailed kinetics description of the system. Furthermore, once the ERN has been extracted from a CFD model, we expect that it can then be extrapolated outside of the original CFD-model conditions for many types of parameter variations, due to the fact that the ERN is a systematic reduction of the physical flow model with realistic kinetics, rather than a parametric fit to the flow-model response. Such extrapolation of the ERN will only be valid for operating conditions that do not greatly perturb the flowfield characteristics. However, this approach has been demonstrated for gasturbine combustor simulations. For example, Drennan, et al. [4] showed that networks of perfectly stirred reactors (PSRs) with detailed chemistry can accurately predict $\mathrm{NO}_{\mathrm{x}}$ emissions over a wide range of fuel/air ratios in industrial gas turbine combustors.

In the work described here, we have developed a general methodology for generating an ERN for a coal gasifier and/or combustor. We start with the assumption that a high-fidelity CFD model can accurately account for the multi-phase transport of condensed-phase particles and gas through the system. Then we apply the ERN-extraction capabilities of ENERGICO software [2] to derive the ERN from the CFD solution. ENERGICO's filtering and extraction algorithms have been extended to account for discretephase material model outputs in the CFD solution. This was needed to adequately partition the gasifier into coherent reacting zones, along with the flowfield parameters, such as flow velocity, residence time, gas composition for major species, and most importantly, temperature. Using these extensions, an ERN can be defined that takes into account the different regions of the gasifier where different chemical processes dominate due to different flow residence times, thermal conditions, and distribution of coal volatiles.

Two example 3-D ANSYS FLUENT models of coal combustion were used to demonstrate the general workflow of building an ERN for a coal combustor/gasifier. The ERN creation process starts with the collection of information on the coal and coal volatiles from the CFD case file and the exporting of the required flow and discrete-phase-material (DPM) solution variables to a CGNS file, using the ANSYS FLUENT user interface. We then devised an automated algorithm for use in Reaction Design's ENERGICO software for partitioning the gasifier/combustor volume and constructing the ERN, where the information from the CGNS file provides the inputs to this procedure. The ERN was run in CHEMKIN-PRO and the results of the ERN simulations were compared with the corresponding CFD simulation outputs, to verify that the ERN behaves well as a reduced-order-model. That model was then also exercised to demonstrate that it can include more chemical kinetics than the original CFD simulation 
and thus produce more information about the gasifier and combustor conditions. In this way we demonstrated that the ERN is more than just a reduced-order-model of the CFD simulation. Finally, we implemented the software interface necessary to incorporate the advanced ROM into a system- or plantlevel process simulation.

For the chemical processing and power-generation industries, process modeling simulators are used to model and simulate the physicochemical processes occurring in chemical plants. An example of such simulation software is Aspen Plus from Aspen Technology [1]. Process simulators represent the chemical processes as a flowsheet diagram, consisting of a number of single Unit Operations (UO) connected to each other by flow streams. Each simulator usually is coupled with its own library of UO models. Examples of such UO models include mixers, splitters, pumps, valves and reactors. In addition to builtin model options, process simulators also often provide one or more interfaces that allow users to insert their own models as UOs within the flowsheet. CAPE-OPEN (CO) [5] includes a standard UO interface designed for interfacing UO components into process simulators. The UO interface provides methods to encapsulate a model based on COM and CORBA protocols. This interface has been adopted by a number of process modeling environments, including Aspen Plus, and are termed "CO simulation environments." A major goal of this project was to take the ROMs developed for the gasifier/combustor processes and encapsulate them such that they could be used seamlessly in CO simulation environments.

For the reactor models employed in Aspen Plus and other process modeling environments, the chemical reactions occurring within the reactor are usually treated in a very simplified manner. Typically, global reactions are used, with just a few chemical steps. Reaction Design's CHEMKIN models, on the other hand, allow very detailed representation of the reaction kinetics within a gas-phase reactor system. CHEMKIN is particularly well established for accurate modeling of gas-surface interactions for catalyzed processes, as well as homogeneous combustion processes. One of the goals of this project was to bring this level of chemical detail into the flowsheet simulation environment, using the CO UO interface provided by Aspen Plus. By including a generalized CHEMKIN model within the CO UO interface of a process modeling simulator, a much more accurate chemistry model can be included to represent gasturbine combustors and coal gasifiers, which will be essential for the design of integrated gasifier/gasturbine combined-cycle systems (IGCC).

To achieve this end, we focused first on the basic reactor representation of a perfectly stirred reactor, then extended this to allow the ROM to consist of a "cluster" of stirred reactors that have interconnecting streams, and finally a ROM that was a fully generalized ERN consisting of multiple PSR clusters with flow connections between them, which are solved iteratively using a tear-stream algorithm. Each level of Unit-Operation encapsulation of the CHEMKIN-based ROMs was carefully validated against the standalone CHEMKIN-PRO interface for the same ERN definition. The ERN ROMs are CHEMKIN-PRO models, where any level of combustion or gas-surface kinetics can be included.

All of the original objectives of the project were met on time and within budget. In this report we summarize the complete workflow from a gasifier CFD model to usage of the derived ROM within a process flowsheet simulation. In addition, we describe the algorithms determined for extracting the ERN from the CFD, the chemistry mechanism used to demonstrate the opportunity for detailed kinetics within the gasifier simulations, the challenges overcome for the flowsheet CAPE-OPEN implementation, as well as the status of the software and an outlook for future development. 


\section{Background}

\subsection{Reactor Networks and ENERGICO ${ }^{\circledR}$ Software}

Researchers have long established that an Equivalent Reactor Network (ERN) can represent a reacting flowfield in a cost-effective and accurate way to account for detailed kinetics and to predict the behavior of a combustor or chemical process, including trace-species emissions. Gas-turbine manufacturers, for example, routinely use perfectly-stirred-reactor (PSR) and plug-flow-reactor (PFR) networks to model gas-turbine combustor behavior, particularly for predictions of $\mathrm{CO}$ and $\mathrm{NO}_{x}$ emission levels, where detailed kinetics are required [6-8]. Kendrick, and coworkers at UTRC [6], for example, showed that networks of perfectly stirred reactors (PSRs) with detailed chemistry can accurately simulate an industrial combustor. They were also able to correctly predict $\mathrm{NO}_{\mathrm{x}}$ emissions over a wide range of fuel/air ratios, combustion efficiency, and the effects of non-mixed regions. In recent years, such approaches have also been applied to industrial-scale coal combustors, furnaces, and boilers [9-12] as well as to bioreactors for multi-phase batch-reactor chemical production [13].

Recognizing the importance and power of the equivalent-reactor-network approach, Reaction Design recently developed and released a product specifically designed to generate, solve, and visualize the results of ERNs, using CHEMKIN-PRO [14] as the solution engine. This product, the ENERGICO® Simulation Package [2], provides flexibility to expert users, while allowing a turn-key approach for nonexperts using established algorithms for extracting reactor networks. The reactor-network simulation is run in CHEMKIN-PRO using state-of-the-art solution techniques for stiff chemistry systems accommodating large reaction mechanisms with 100s of species and 1000s of reactions. The reactor network simulation within CHEMKIN-PRO allows for complex flow-connectivity between idealized reactors (PSRs and PFRs), including upstream flow connections, making use of a tear-stream solution algorithm. A schematic of the workflow facilitated by ENERGICO is shown in Figure 1.

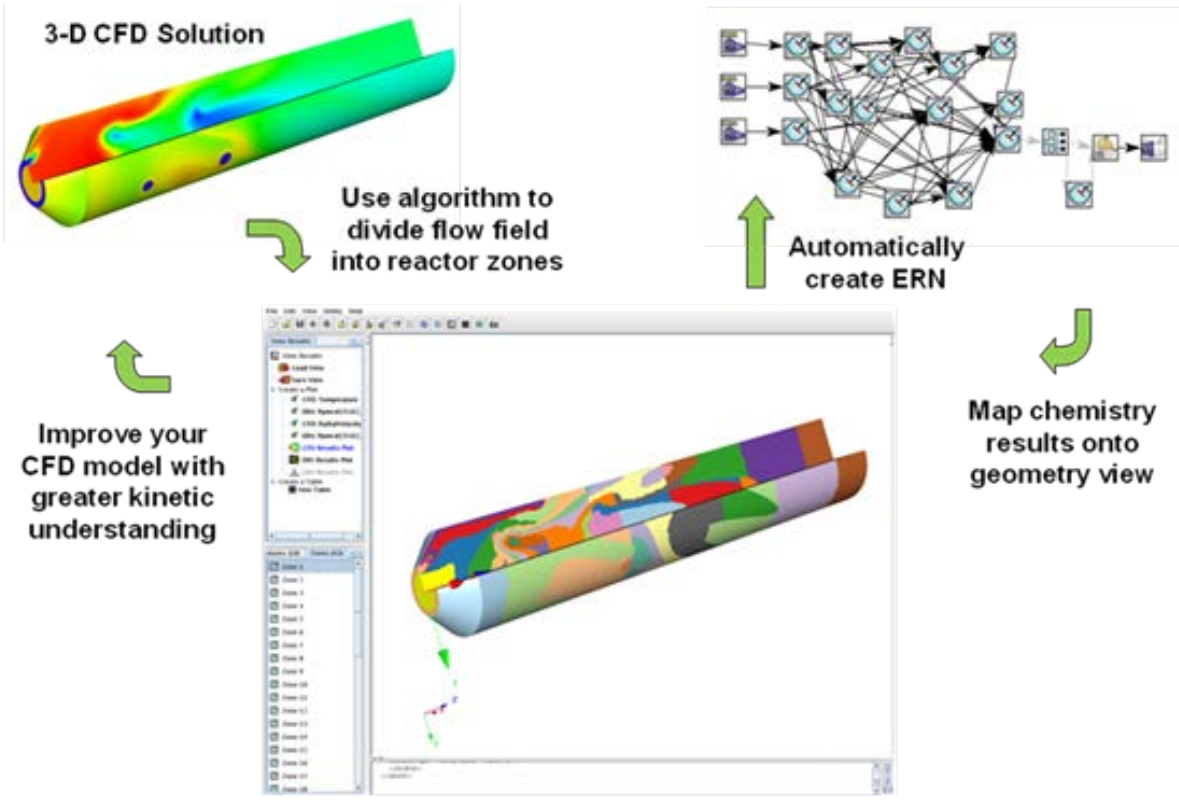

Figure 1. Workflow for creating a reactor network from a CFD solution within the ENERGICO Simulation Package. 
This approach has proven to be very effective in predicting ppm-level $\mathrm{NO}_{\mathrm{x}}$ and $\mathrm{CO}$ emissions from gasturbine combustors, using high-fidelity models of the combustor geometry in CFD and high-fidelity chemistry models in the ERN solution for the gas-phase combustion and emissions production. Furthermore, once the ERN is established, studies of fuel loading effects on emissions can be performed efficiently and accurately without repeated simulation of the computationally expensive CFD solution. This is due to the fact that the ERN retains all the dominant physics and flow relationships of the original CFD solution, even while adding detailed kinetics. Such a physically based model can predict outside of the original set of conditions much more reliably than a model that has simply been fit to data or to a more complex model's results.

This ENERGICO/CHEMKIN-PRO reactor-network capability formed the basis of the work reported here, where one goal was to extend this concept to be applicable to entrained-flow gasifiers. In addition, in order to gain as much extra benefit from the ERN approach, attention was paid to the development of a more detailed description of the gasifier kinetics than is typically included in CFD simulations.

\subsection{CAPE-OPEN Architecture}

In addition to extending the ERN concept to gasifier applications, the other main objective of the project was to package a general ERN, representing a complex flowfield and including detailed kinetics, within a process flowsheet simulator using the CAPE-OPEN Unit Operation interface to the flowsheet. The CAPEOPEN (CO) Unit Operation (UO) interface specification is well documented by Co-LaN [5]. However, several implementation decisions had to be established during the project. These included:

- Programming Language for the Implementation. At the beginning of this project, the only language supported for implementation of a Unit Operation (UO) by the Co-LaN organization was VB6. Already unsupported by Microsoft and arguably obsolete, working in VB6 proved challenging in terms of code robustness and compatibility with other code components. Fortunately, Reaction Design's interaction with the Co-LaN organization helped push the support of $\mathrm{C}++$ based UO coding, which was adopted for this project.

- Packaging Strategy. The COM interface was selected for the CO implementation, since the targeted process flowsheet simulator, Aspen Plus, is only available on Windows 32-bit platforms. We further decided to package the UO as a generic, single dynamically linked library (DLL), which could read-in the specific ERN settings at the time of instantiation in the flowsheet simulator.

- Encapsulation of the Complex ERN into a Single Unit Operation. In principle, the process flowsheet simulator already includes a tear-stream algorithm for iterative solution of the flow traversing multiple units, with upstream as well as downstream connections. One option considered, then, was to only package single PSRs or PSR-Clusters and somehow use the flowsheet iteration method. However, given the high efficiency established in the solution of the ERNs within the CHEMKIN-PRO framework, and the unique ability to handle complex kinetics that would not be well handled in the flowsheet simulation, we decided early that this was not a viable option. Instead, we took on the task of packaging the full ERN created by ENERGICO as a single UO, and modifying the solution and Input/Output (I/O) architecture to enable that encapsulation.

These decision points comprised some of the major milestones for the project. In addition, we took a step-by-step approach to the implementation to build up our experience with the CO interface and the flowsheet simulators. The main milestones of this step-by-step approach were the implementation of a single PSR, a cluster of PSRs, and then the full ERN that included a tear-stream solution component. We 
employed both AspenTech's Aspen Plus simulator as well as the Co-LaN organization's COFE simulator. The COFE simulator was employed to get around some bugs found in the AspenTech CO implementation, as well as to assure that we kept to the generic CO interface without inadvertently employing any of the specialized features of the Aspen Plus implementation.

\subsection{Kinetics Models for Coal Gasification}

The application focus for this project was on Entrained Flow Gasifiers, which are the dominant form of gasifier in use for Integrated Gasification Combined Cycle (IGCC) systems used by key manufacturers such as ConocoPhillips, GE Energy and Shell Global. For these systems, a dry pulverized solid or slurry is typically gasified with oxygen in a co-current flow. The flowfield involves a dense cloud of very fine fuel particles, in which the gasification reactions take place. Gasification processes include devolatilization, pyrolysis and heterogeneous reactions on the particle surface, as well as gaseous reforming reactions. These processes are made more complex by the dependence on the type and source of coal.

While Reaction Design's ENERGICO [2] software has been established and validated for simulating emissions of modern gas-turbine combustors used in energy generation and transportation, with accurate predictions at ppm levels for $\mathrm{NO}_{\mathrm{x}}$ and $\mathrm{CO}$, the application of this software to the prediction of coalgasifier performance required extensions to the technology. Specifically, we had to address a variety of complex phenomena, including:

- Early particle devolatilization, pyrolysis, and reaction

- Char particle transport and burn out

- Gas-phase reaction mechanisms for pyrolysis and reforming

- Interactions with the slag (molten ash) within the gasifier

While addressing all of these processes in detail is an ambitious undertaking, our aim in the context of this project was mainly to create a framework where such studies could be performed using more rigorous chemistry descriptions.

At the start of this project, Reaction Design had already assembled well-validated mechanisms for gasphase combustion of a large array of hydrocarbon fuels, which have been tested under pyrolysis and oxidation conditions for a wide range of temperatures and pressures [15-18]. These mechanisms include well-validated $\mathrm{NO}_{x}$-formation submechanisms that cover both low- and high-temperature conditions and that incorporate recent work in fuel-NO reactions and mutual combustion sensitization [19, 20]. In addition, we had ongoing mechanism-development work in the area of soot-particle formation, which made use of the CHEMKIN Particle Tracking module [14]. The Particle Tracking module determines particle-size distributions under particle growth and oxidation conditions while considering the heterogeneous reaction kinetics at the gas/particle-cloud interface, in the context of CHEMKIN reactingflow models. This general-kinetics capability allowed testing of competing reaction pathways for particle oxidation and growth to determine dominant effects under different flow conditions.

Initial kinetics-model components were assembled from recent works focused on coal devolatilization, char combustion and char gasification kinetics, including both detailed kinetics studies under idealized conditions and more empirical models tested in the context of CFD simulations. A review of some of the kinetics models relevant to entrained-flow gasifiers is provided by Bockelie, et al. [21]. Other works report a multi-step kinetics-based model of char oxidation during pulverized coal combustion [22] as well as global reaction expressions for the char combustion that account for different coal characteristics [23]. Using the general kinetics capabilities of CHEMKIN, we tested the concepts represented by different levels of chemistry modeling and from this designed an implementation for a general-purpose kinetics 
model in the CHEMKIN framework. A combination of homogeneous and heterogeneous reaction mechanisms were considered, employing the general surface-chemistry capability and particle sizedistribution modeling in the CHEMKIN-based simulation. 


\section{Methodology}

\subsection{Workflow for Creating and Using ERN-Based ROMs}

During this project, Reaction Design's ENERGICO software was extended to create ERNs for use in prediction of coal gasifier performance. In addition, these ERNs were then packaged in a general way to allow use within the process flowsheet simulator, Aspen Plus. The top-level workflow for creating and using ERNs as ROMS within an Aspen Plus simulation is as follows:

1. Obtain a CFD model of the gasifier / combustor equipment that has been successfully run.

2. Export the CFD solution into a commonly supported file format, called CGNS.

3. Import the CFD CGNS solution file into the ENERGICO software.

4. Within ENERGICO, apply an algorithm to generate the ERN.

5. Within the ENERGICO/CHEMKIN-PRO companion interfaces, view the ERN, run and validate the model, and view results in the context of the 3-D geometry. Compare with the original CFD to make sure it is a good representation of the model.

6. When ready to use the ERN ROM model within Aspen Plus, use the export option within CHEMKIN-PRO interface to export the ERN to a CHEMKIN CAPE-OPEN descriptor file.

7. From within Aspen Plus, enable the CHEMKIN-CO DLL by adding the corresponding Unit Operation to the flowsheet. Connect the inlet and outlet ports to the CHEMKIN-CO object and allow the object to read in the exported descriptor file. Identify species mapping as needed.

8. Run the Aspen Plus flowsheet simulation.

Each of these steps are described in more detail below, along with some details on the implementation and simulation approach within enable each step.

\subsubsection{Exporting the CFD Solution for Use in ENERGICO}

The starting point of the workflow for creating reactor networks is to read in a CFD solution that represents the geometry and flow of the gasifier component. The solution contains flow velocities, temperatures, and particle distribution information or discrete-phase material (DPM) source terms, depending on the CFD model's capabilities. To allow representation of a coal gasifier, we extended the ENERGICO software to read in particle and/or DPM information and to transfer relevant source information to the reacting-flow simulation in CHEMKIN-PRO. In this section we describe the workflow enabled by these modifications.

To develop a process, we first acquired several ANSYS FLUENT® [24] CFD models that represent coal combustion and gasification from NETL and from Ansys, Inc. CFD simulations of coal combustion can provide spatial distributions of coal particles based on the bulk-flow convection and turbulence dissipation. All of the CFD cases we considered employed the Discrete Phase Model (DPM) available in ANSYS FLUENT to represent the coal gasification sources and sinks. We focused our initial efforts on the relatively simple coal-combustion case from FLUENT tutorials. From this case, we confirmed that source/sink terms of gas species involved in the coal combustion and gasification can be exported to the solution file in the CGNS format required by ENERGICO. 
The availability of DPM source terms gave us the flexibility to perform the ERN coal simulations in one of two ways:

1. Use the DPM gas-species source terms from the CFD solution directly in the ERN simulation. This method does not require a coal (or particle) model in the reactor-network simulation. However, a mapping between CHEMKIN species and both the CFD gas-phase and the CFD DPM volatile species was needed. Use of this option means that the ERN simulation relies on the CFD models for prediction of:
a. Coal devolatilization.
b. Char combustion (usually a one- or two-step global reaction).
c. Particle size distribution.
d. Turbulence-chemistry interactions.

The CHEMKIN-based ERN, then, will use these CFD predictions as inputs and then predict the gas-phase products using high-fidelity kinetics models for the pyrolysis, reforming, and pollutant formation processes in the gasifier. However, the coal-devolatilization rates remain fixed to the values derived from the CFD solution.

2. Use the CFD model prediction of gas-phase species and discrete-phase-material concentrations at external inlets to the gasifier only. The flow solution and other information from the CFD results would then be used mainly as criteria to determine the ERN structure, but the devolatilization and char-combustion results are not used directly as source/sink terms in the ERN calculation. Instead, the devolatilization and combustion of coal particles would be handled by the ERN coal model, including particle size tracking as particles combust. This method assumes that the coal particles always travel with the bulk flow and will have the same split ratio to downstream reactors as the bulk gas flow. However, this approach allows us to expand on the coal devolatilization and combustion kinetics models and compare simpler vs. more complex formulations, in addition to introducing the high-fidelity gas-phase chemistry.

Both of these methods rely on the details of the CFD solution to determine the structure of the reactor network. For this reason, we focused initially on the first approach for the development of the ERNextraction algorithm.

By utilizing the DPM variables from the CFD solution, we were able to use ENERGICO to partition the gasifier/combustor volume into zones that will be further translated into an ERN. Figure 2 gives an example of zone partitions based on the DPM mass source inside the coal gasifier/combustor. The zones can be refined further by applying other filters based on, for example, local temperature and axial location, as discussed further below. 


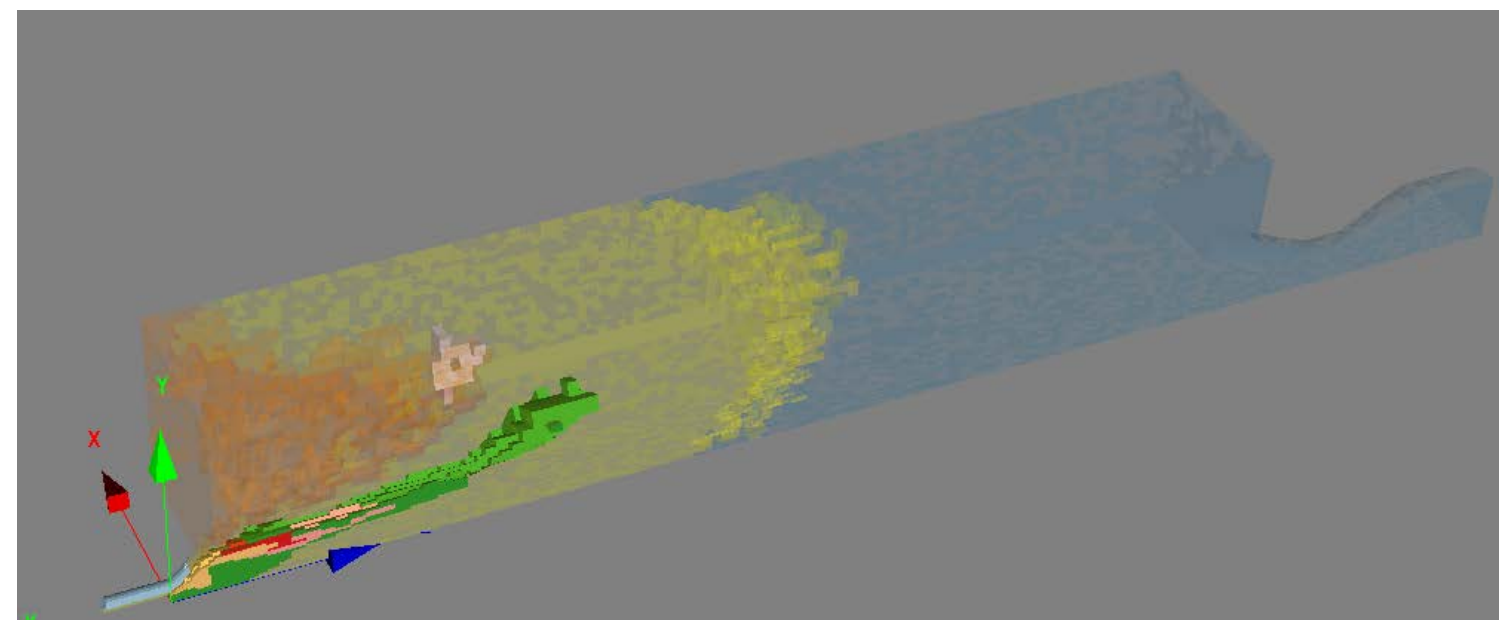

Figure 2. Zonal partitions of a coal combustor based on DPM mass source.

\subsubsection{Representing Coal Volatiles in the ERN's Detailed Chemistry}

Coal can be divided into three main functional components: volatile, combustible (fixed carbon/char), and ash. The weight fraction of each component is obtained from the coal proximate analysis. The elemental makeup of the volatile component, on the other hand, can be derived from the ultimate analysis data. Coal volatiles are commonly represented by a pseudo species in FLUENT DPM simulations (see Figure 3). The single pseudo volatile species in the CFD simulations represents collectively the average elemental makeup of all volatiles in the coal and often cannot be mapped to a single chemical gas-phase species in the reaction mechanism that is employed in the ERN. We therefore developed a scheme that allows us to use a mixture of gas species to characterize the pseudo volatile species. The scheme requires information about the elemental composition of the coal volatile species, which can be obtained from the coal analysis data or from the CFD case directly.

The lone pseudo volatile species assumes the average elemental makeup and molecular weight of real coal volatiles. The devolatilization of the coal volatiles is often given as a single-step reaction, yielding stable products such as $\mathrm{H}_{2} \mathrm{O}, \mathrm{CO}_{2}$, and $\mathrm{N}_{2}$ (see Figure 3). Consequently, any contribution of coal volatiles, through intermediate species, to other gas-phase chemical processes, such as fuel pyrolysis and pollutant formation, will not be considered in the CFD simulations.

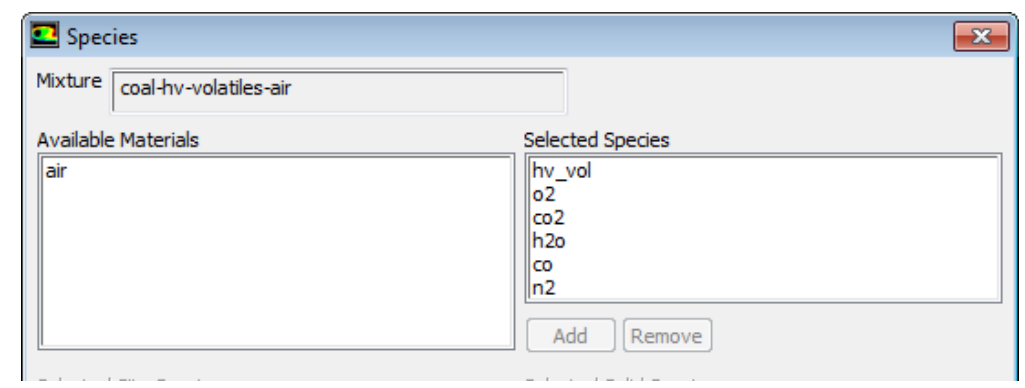

Figure 3. The "Species" panel in FLUENT showing the pseudo coal volatile species $h v_{-} v o l$.

A preparatory task must be performed prior to building the ERN coal gasifier model in ENERGICO, so that the pseudo coal volatile species and its influences can be fully incorporated into the detailed gasphase reaction mechanism used in the ERN. A group of gas-phase species must be selected from the detailed reaction mechanism (used by the ERN) to represent the coal volatiles. The choice of these gas species is somewhat arbitrary, but to arrive at the set used here, we invoked the following principles: 
(1) the gas species composition should comprise the same overall elemental composition as the volatiles and (2) the gas species should participate in reaction pathways that reasonably characterize reactions involving coal volatiles. Once the candidate species are selected, if the number of gas components in the mixture representing the coal volatiles equals the number of elements in the coal volatiles, the species fractions can be uniquely determined by the conservation of elements.

The elemental composition of the coal volatiles can be obtained from the coal ultimate-analysis data. If there were no volatile data available for the coal, the devolatilization reaction given in the "Reactions" panel in the FLUENT model could be used instead to convert the pseudo-coal volatile species from the CFD model to a gas-phase species in the CHEMKIN mechanism. An example of a single-step coal volatilization reaction in FLUENT is shown in Figure 4.

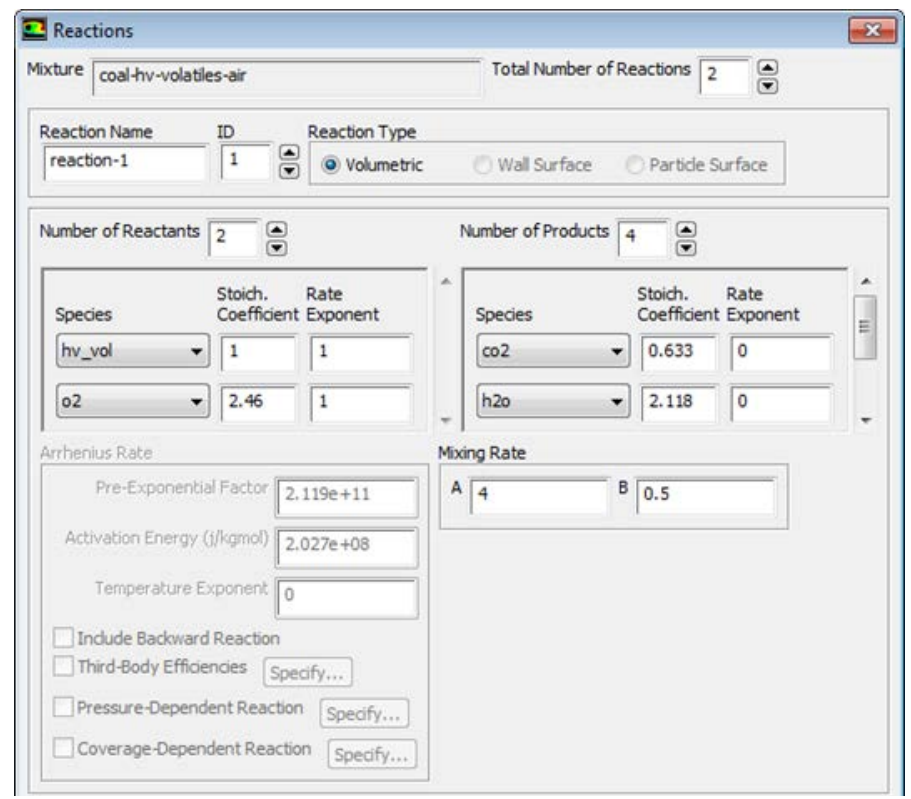

Figure 4. FLUENT "Reactions" panel showing products and stoichiometric coefficients of the coal devolatilization reaction.

The process of deriving the coal volatile elemental composition from the FLUENT devolatilization reaction is straightforward. First, we find the reaction that involves the pseudo-coal volatile species as in the FLUENT reaction mechanism. The elemental composition of the pseudo-coal volatile species can then be back-calculated from the stoichiometric coefficients of this "volatile decomposition/combustion" global reaction. For example, assuming the formulation of the pseudo-coal volatile species, $h v \_v o l$ is given as $\mathrm{C}_{a} \mathrm{H}_{b} \mathrm{O}_{c} \mathrm{~N}_{d} \mathrm{~S}_{e}$, the devolatilization reaction can be re-expressed as

$$
\mathrm{C}_{a} \mathrm{H}_{b} \mathrm{O}_{c} \mathrm{~N}_{d} \mathrm{~S}_{e}+2.46 \mathrm{O}_{2}=>0.633 \mathrm{CO}_{2}+2.118 \mathrm{H}_{2} \mathrm{O}+2.17 \mathrm{CO}+0.071 \mathrm{~N}_{2}
$$

The element fractions in the pseudo-volatile species can be determined by conserving the elements of the devolatilization reaction:

$$
\begin{aligned}
& a=0.633+2.17=2.803 \\
& b=2.118^{*} 2=4.236 \\
& c=0.633^{*} 2+2.118+2.17-2.46^{*} 2=0.634 \\
& d=0.071^{*} 2=0.142 \\
& e=0.0
\end{aligned}
$$


Thus the formulation of the pseudo-coal volatile species becomes $C_{2.803} \mathrm{H}_{4.236} \mathrm{O}_{0.634} \mathrm{~N}_{0.142}$. The molecular weight of the volatile, $\mathrm{W}_{v o l}$, is $50.004[\mathrm{~g} / \mathrm{mole}]$.

We used Reaction Design's C2_NO ${ }_{x}$ mechanism [25] in our testing, so that we could explore both coal volatile oxidation and $\mathrm{NO}_{\mathrm{x}}$ formation (via prompt, thermal, and fuel-nitrogen pathways). Since there are four elements in the pseudo volatile species, the representative "volatile mixture" can be uniquely determined with four gas-phase species. The individual gas species do not have to each contain all four elements, as long as all elements are present in the resulting mixture. Here our model of "volatile mixture" consists of $\alpha_{a}$ moles of $\mathrm{C}_{2} \mathrm{H}_{4}, \alpha_{b}$ moles of $\mathrm{H}_{2}, \alpha_{c}$ moles of $\mathrm{CO}$, and $\alpha_{d}$ mole of $\mathrm{HCN}$; that is,

$$
\mathrm{C}_{2.803} \mathrm{H}_{4.236} \mathrm{O}_{0.634} \mathrm{~N}_{0.142}=\alpha_{a} \mathrm{C}_{2} \mathrm{H}_{4}+\alpha_{b} \mathrm{H}_{2}+\alpha_{c} \mathrm{CO}+\alpha_{d} \mathrm{HCN} .
$$

The choice of $\mathrm{C}_{2} \mathrm{H}_{4}$ as the hydrocarbon volatile is based on the $\mathrm{C}-\mathrm{H}$ ratio of the pseudo-volatile species and the desire to represent the volatiles with kinetically stable compounds. The conversion of fuel nitrogen during coal combustion and devolatilization is usually rapid and complete. The major gas species generated from fuel nitrogen conversion are hydrogen cyanide $(\mathrm{HCN})$ and ammonia $\left(\mathrm{NH}_{3}\right)$. Hydrogen cyanide is the principal product when most fuel nitrogen is bound in aromatic rings [26]. If most fuel nitrogen is in the form of amines, the main product will be ammonia, or possibly amidogen $\left(\mathrm{NH}_{2}\right)$.

The number of moles of each gas component is computed by invoking conservation of the elements:

$$
\begin{aligned}
& { }_{2} \alpha_{a+} \alpha_{c+} \alpha_{d}=2.803 \\
& { }_{4} \alpha_{a+2} \alpha_{b+} \alpha_{d}=4.236 \\
& \alpha_{c}=0.634 \\
& \alpha_{d}=0.142 \\
& \alpha_{a}=1.0135 \\
& \alpha_{b}=0.02
\end{aligned}
$$

Since species variables are usually reported as mass fractions in the CFD solution, we must convert the molar composition obtained using the above relations to mass fractions, using the molecular weights of each of the gas species. For example, the mass fraction of species component $k$ of the volatile mixture can be computed as:

$$
\alpha_{k}^{\prime}=\alpha_{k} W_{k} / W_{v o l}
$$

where $W_{k}$ is the molecular weight of gas species $k$, and $W_{k}$ is the molecular weight of the volatile species.

With the mass fractions defined, we can then parse out the devolatilization source terms into corresponding gas-phase source terms. For example, if the devolatilization rate is $\dot{g}_{v o l}[\mathrm{~g} / \mathrm{s}]$, the mass production rates of the "volatile mixture" components are given as

$$
\begin{aligned}
& \dot{\boldsymbol{g}}_{C 2 \mathrm{H} 4}=\alpha_{a}^{\prime} \dot{\boldsymbol{g}}_{\mathrm{vol}}[\mathrm{g} / \mathrm{s}] \\
& \dot{\boldsymbol{g}}_{\mathrm{H} 2}=\alpha_{b}^{\prime} \dot{\boldsymbol{g}}_{\mathrm{vol}}[\mathrm{g} / \mathrm{s}] \\
& \dot{\boldsymbol{g}}_{C O}=\alpha_{c}^{\prime} \dot{\boldsymbol{g}}_{\mathrm{vol}}[\mathrm{g} / \mathrm{s}] \\
& \dot{\boldsymbol{g}}_{\mathrm{HCN}}=\alpha_{d}^{\prime} \dot{\boldsymbol{g}}_{\mathrm{vol}}[\mathrm{g} / \mathrm{s}]
\end{aligned}
$$


These relationships are to create user-defined variables when we map the DPM mass production rate to individual species mass production rates within the ENERGICO user interface.

The ANSYS FLUENT User's Guide states that the Burnout Stoichiometric Ratio is used in the calculation of the diffusion-controlled burnout rate but has no impact on the chemistry. The Burnout Stoichiometric Ratio is the mass of oxidant required per mass of char. The default value of $2.67\left(W_{\mathrm{O} 2} / \mathrm{W}_{\mathrm{C}}=32 / 12\right)$ assumes that carbon atoms in char are oxidized by $\mathrm{O}_{2}$ to yield $\mathrm{CO}_{2}$ (see Figure 5). A Burnout Stoichiometric Ratio of 1.33 indicates $\mathrm{CO}$ being the char combustion product. For a burnout rate reported by the DPM model as $\dot{g}_{\text {burnout }}[\mathrm{g} / \mathrm{s}]$, the corresponding $\mathrm{CO}_{2}$ production rate due to char oxidation is then:

$$
\dot{g}_{C O 2,0 x}=\dot{g}_{\text {burnout }}[g / s]
$$

and the $\mathrm{O}_{2}$ consumption rate (negative value) is

$$
\dot{g}_{\mathrm{O}, \mathrm{ox}}=-\left(W_{\mathrm{O} 2} / W_{\mathrm{CO} 2}\right) \dot{g}_{\text {burnout }}[\mathrm{g} / \mathrm{s}] \text {. }
$$

The total mass production rate of a gas species $k$ due to both coal devolatilization and char burnout can then be determined as:

$$
\dot{g}_{k}=\dot{g}_{k, v o l}+\dot{g}_{k, o x} .
$$

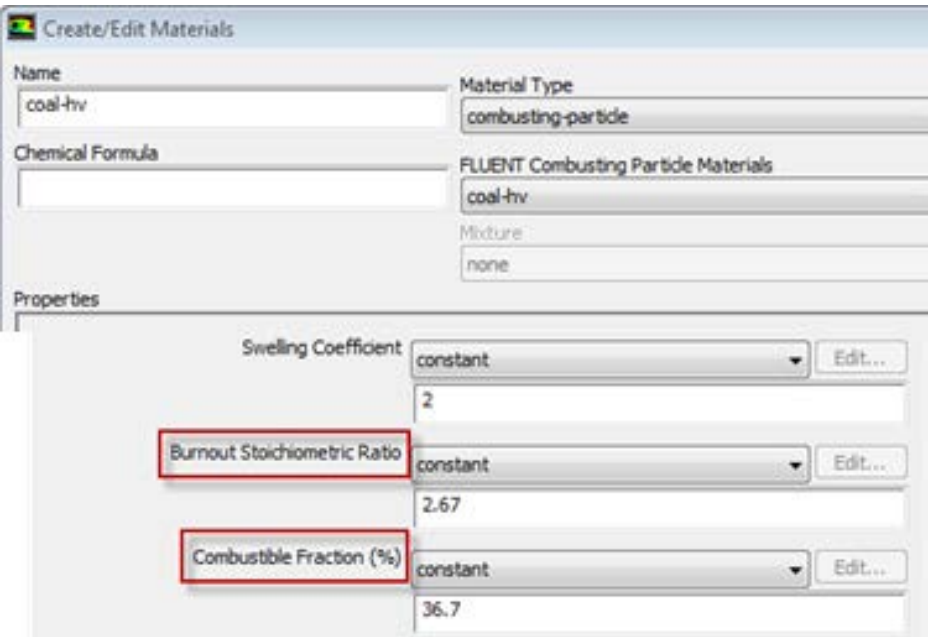

Figure 5. The FLUENT "Material" panel showing the coal burnout model parameters.

\subsubsection{Performing Zone Partitioning within ENERGICO to Define the ERN}

The ENERGICO software enables the partitioning of the reactor volume into zones by applying filters that are defined by variable values in the CFD solution file. For example, you can separate the volume based on grouping cells that have similar ranges of CFD cell temperatures, using a temperature filter. In this way, any quantities that could be used to characterize the application should be exported to the CGNS solution file for consideration in ENERGICO. Some CFD variables are always required and they are: geometry (coordinate information), velocities, static temperature, pressure, species mass fractions and mass production rates of species. Variables that relate to sub-models in the CFD simulation, such as turbulence kinetic energy, may also be useful but are optional. For coal combustion and devolatilization applications, DPM concentrations and mass source/sink terms are required. The DPM mass source/sink terms are especially important, because the current modeling approach relies on these source/sink terms to capture the chemical interactions between the coal and gas. 


\section{Determining What CFD Flow Variables Are Needed for Export from the CFD Solution}

There are some CFD solution variables that are always important for creating an ERN. Because combustion chemistry is highly temperature sensitive, static temperature is critical to providing good resolution in the flame zone (for a combustor) as well as to separating different reaction pathways that may occur in different temperature ranges. For example, the thermal De-NOx process, in which ammonia is injected into the exhaust gas to reduce NOx, works only in a narrow temperature window between $1100 \mathrm{~K}$ and $1400 \mathrm{~K}$ [26]. The relatively cool boundary zones next to the combustor liner are also important since they could keep local $\mathrm{CO}$ from further oxidation into $\mathrm{CO}_{2}$. The static gas temperature contours in a coal combustor are shown in Figure 6, in which the flame zone and some cool gas pockets next to the top wall can be seen. Species concentrations (or mass/mole fractions) can be used to isolate regions of chemistry activity or inactivity. Species that are distinctly presented in the inlet streams can serve as excellent criteria for extracting entrance regions from the rest of the reactor volume, so that reactants from different inlets will not be mixed prematurely.

Velocities are also important for defining an ERN, because they determine local flow residence times, which are also an important factor in determining the dominant chemical kinetics in a region. The velocity contours (shown for our sample problem in Figure 7 and Figure 8) can be used as filter criteria to identifying recirculation regions inside the reactor. The separation of recirculation zones from the main downstream-flow regions can allow the flame stabilization mechanism to be isolated in the ERN structure. The coordinate in the flow direction can be used subsequently to divide the post-flame region into a number of smaller zones, such that the plug-flow-like characteristics of this region can be appropriately modeled by a series of PSRs.

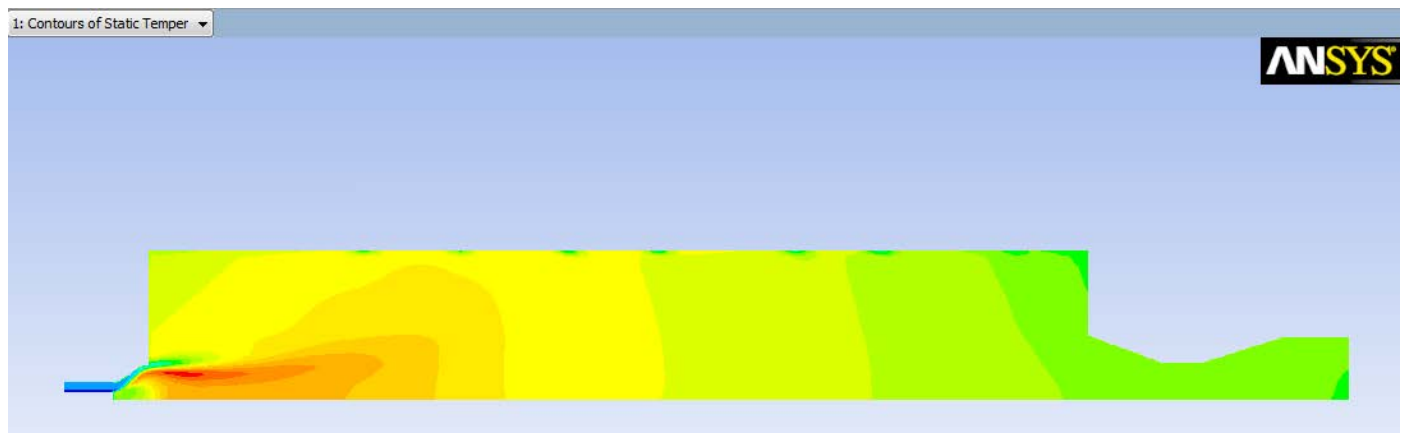

Figure 6. Static temperature contours in a coal combustor.

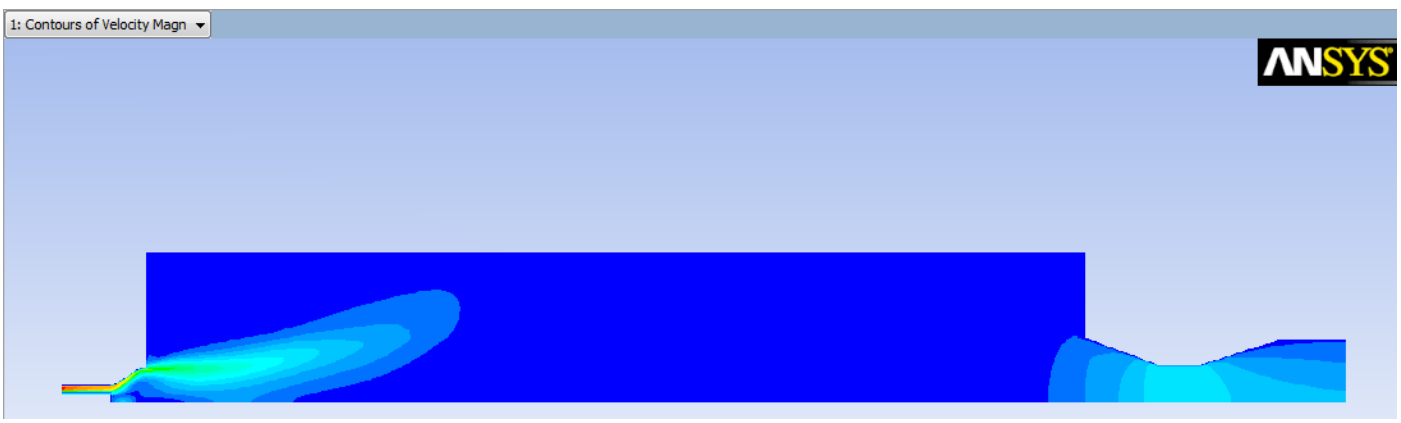

Figure 7. Axial velocity contours in the coal combustor. 


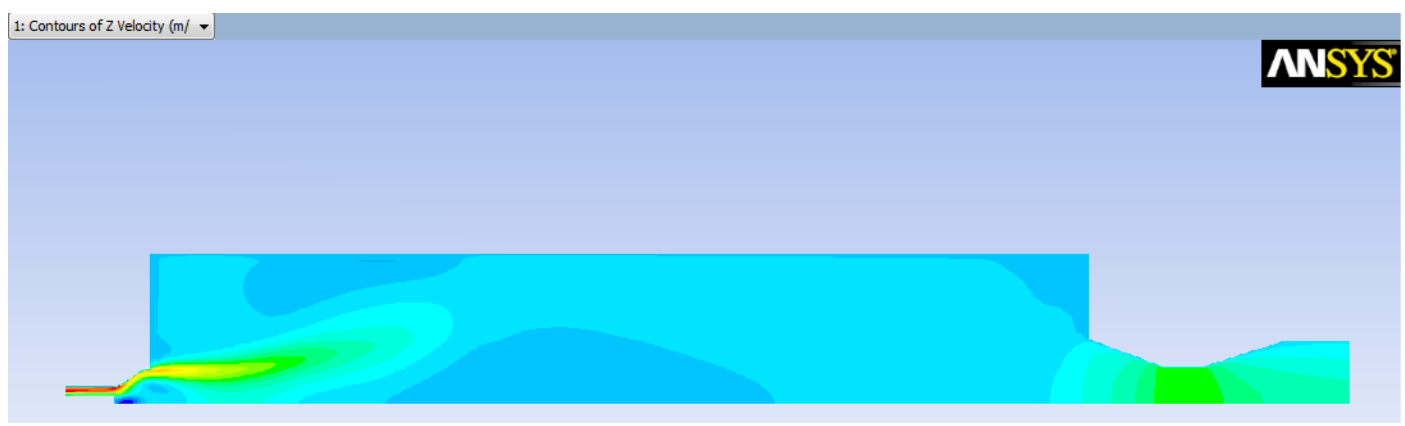

Figure 8. Transverse velocity contours in the coal combustor.

When a FLUENT CFD simulation involves the Discrete Phase Model (DPM), DPM variables, such as DPM concentration, become available to be exported to the CGNS solution file, in addition to the more common CFD variables. These DPM variables can be used in ENERGICO to extract regions where certain surface-gas interactions dominate, such as devolatilization or burnout. The DPM source/sink terms in the CFD solution also provide a simple method for including the surface-gas interactions in the ERN simulations, when a detailed coal devolatilization model is not available. The DPM concentration distribution given in Figure 9 does not always indicate the full extent of the region with coal-gas interactions. As shown in Figure 10, the DPM mass source distribution, which is the sum of DPM mass sources from devolatilization and burnout, is a better indicator for identifying regions with coal-gas activities. DPM mass source terms for coal volatile or char burnout species, as shown in Figure 11, Figure 12 and Figure 13, can be used to further separate regions with different coal-gas interaction processes.

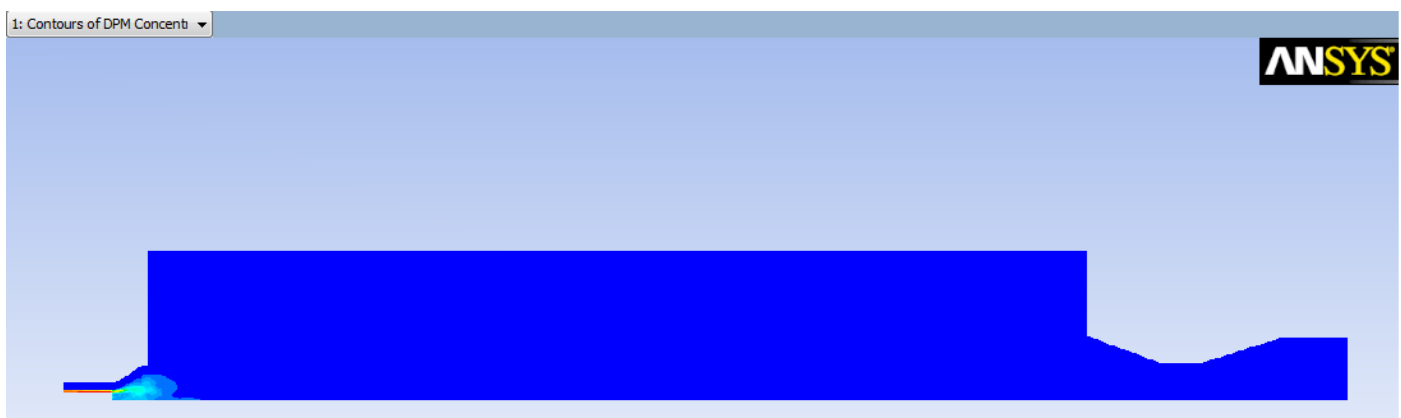

Figure 9. Contours of DPM concentration in a coal combustor.

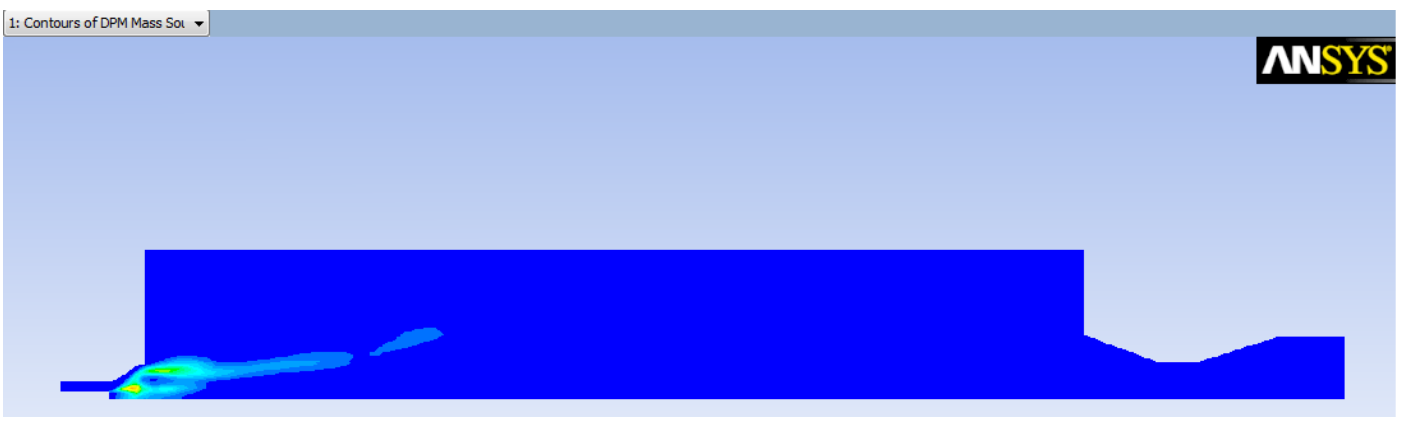

Figure 10. DPM mass source distribution indicates the combustor region having noticeable coal-gas interaction. 


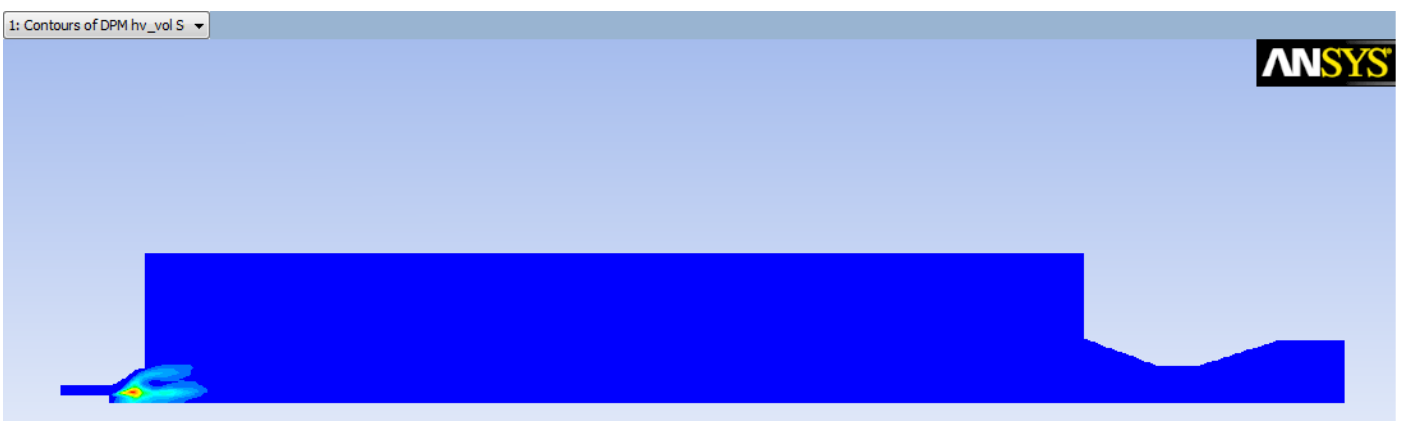

Figure 11. DPM mass source contours of pseudo coal volatile species $h v_{-} v o l$ identifying combustor region with coal devolatilization activities. Note that the DPM $h v_{-} v o l$ source term is the same as the DPM devolatilization mass source in FLUENT.

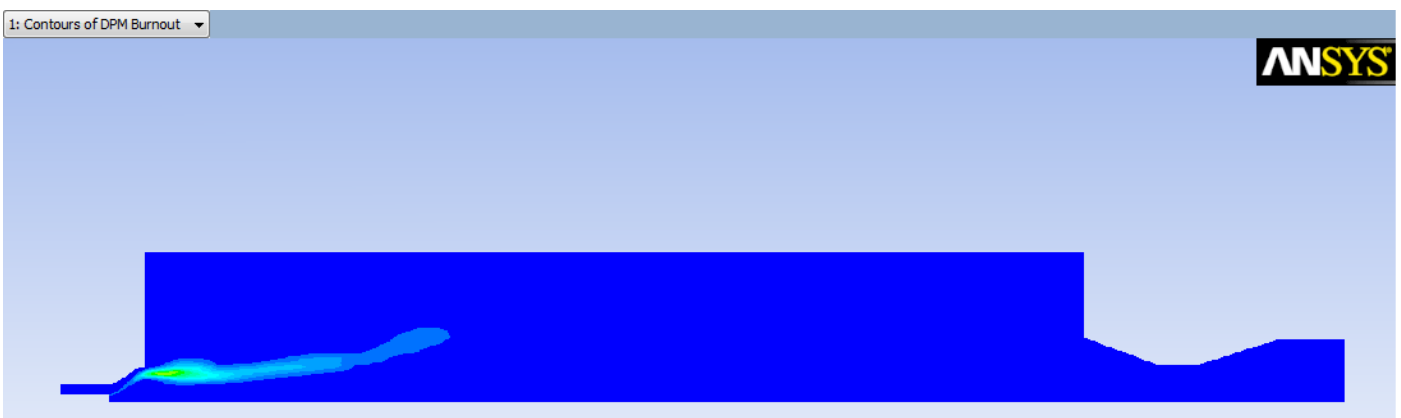

Figure 12. DPM burnout mass source contours identifying the combustor region with char burnout activities.

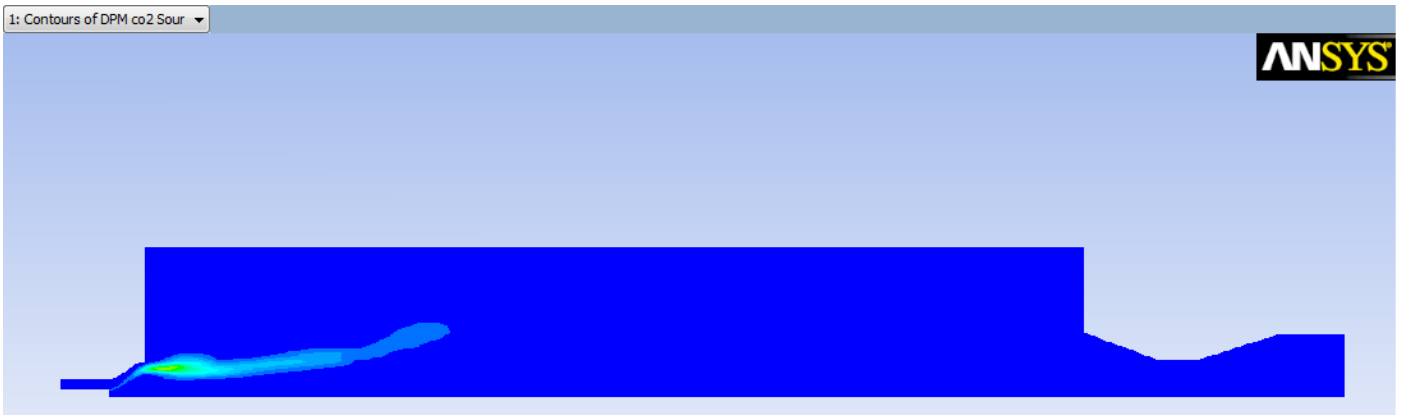

Figure 13. DPM mass source contours of char burnout product $\mathrm{CO}_{2}$ identifying combustor region with coal devolatilization activities. Note that the DPM $\mathrm{CO}_{2}$ source term is the same as the DPM burnout mass source given in Figure 12.

\section{Exporting Desired Information from the CFD Solution File}

Once solution variables necessary for ERN creation are identified, we use the export feature provided by the CFD software to export the desired information into a CGNS-formatted file. All DPM-related solution variables are prefixed with the term "DPM" in FLUENT and can be selected in the "Quantities" list on the "Export" panel as shown in Figure 14. 


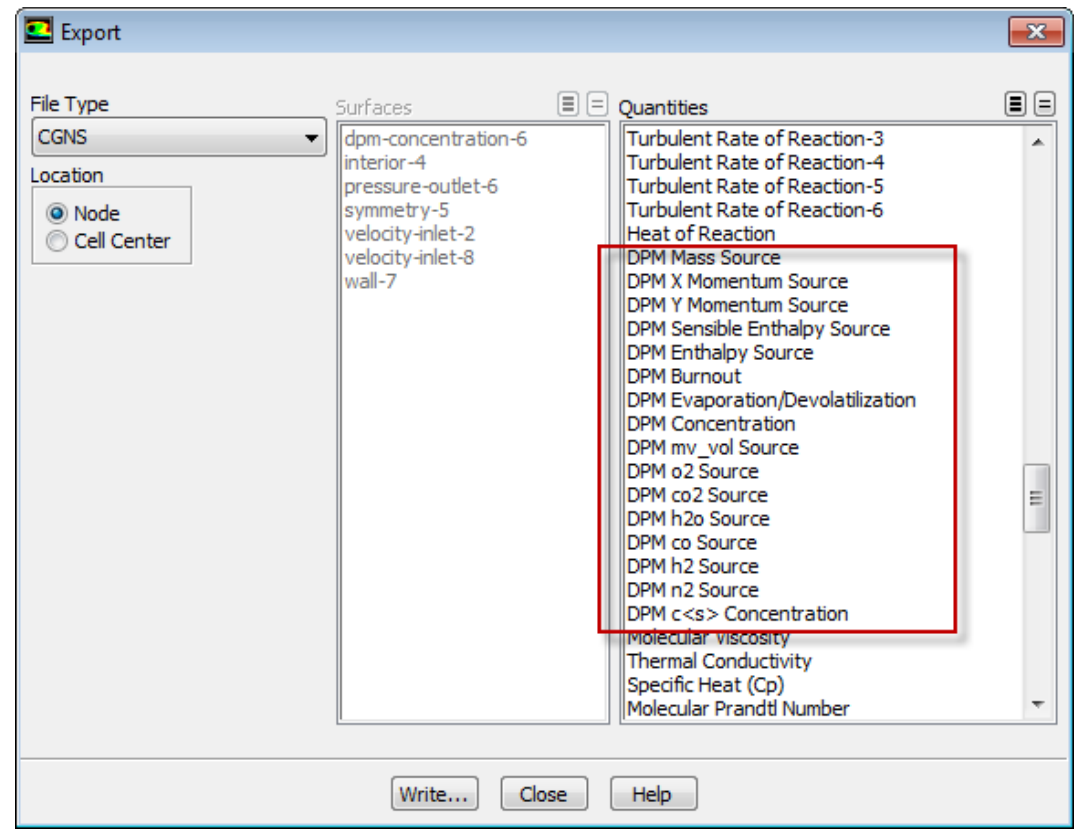

Figure 14. List of Discrete Phase Model (DPM) solution variables that can be exported from FLUENT and utilized by ENERGICO to create ERN for coal gasifier simulations.

\section{Importing the CFD Solution into ENERGICO}

A session in ENERGICO starts with the import of the CGNS-formatted CFD solution file that was exported from the CFD software's user interface. The most common CFD solution variables, such as coordinates, velocities, and temperature and boundary conditions, are automatically recognized and processed by ENERGICO. General instructions for importing CGNS files into ENERGICO and other general operations can be found in the ENERGICO User Manual [2]. For coal gasifier/combustor applications, certain additional steps are necessary.

\section{Defining Custom Variables for Coal-Related Simulations}

Once the solution file is imported into ENERGICO, we need to address the issue of the pseudo volatile species employed by the DPM. Detailed description of the volatile mapping scheme is provided in Section 3.1.2. Within ENERGICO, the issue of representing a single pseudo-volatile gas species in the CFD solution with a gas mixture in the CHEMKIN mechanism is addressed by the creation of userdefined variables. In this case, the pseudo-volatile gas species is modeled by a mixture of 4 gas species in the CHEMKIN gas reaction mechanism: $\mathrm{C}_{2} \mathrm{H}_{4}, \mathrm{H}_{2}, \mathrm{CO}$, and $\mathrm{HCN}$. For our sample case, the actual composition of the coal volatile is determined from the "devolatilization reaction" used in the FLUENT simulation

The corresponding volatile gas mixture composition is listed in Table 1.

Table 1. Mass fractions of coal volatile gas mixture components.

\begin{tabular}{|c|c|}
\hline Gas Species in Volatile Mixture & Gram per Gram Coal Volatiles \\
\hline $\mathrm{C}_{2} \mathrm{H}_{4}$ & 0.5675 \\
\hline $\mathrm{H}_{2}$ & 0.0008 \\
\hline $\mathrm{CO}$ & 0.3550 \\
\hline $\mathrm{HCN}$ & 0.0767 \\
\hline
\end{tabular}


Figure 15 shows the user-defined $\mathrm{CO}$ mass fraction variable $\mathrm{YCO}$ and the formula that relates $\mathrm{CO}$ mass fraction to the corresponding DPM variables in the CFD solution. The CO mass fraction in the volatile gas mixture (or the constant multiplier in the definition) is determined by the conservation of the elements, as previously discussed. A similar user-defined mapping is created for each of the 4 species representing the volatile mixture.

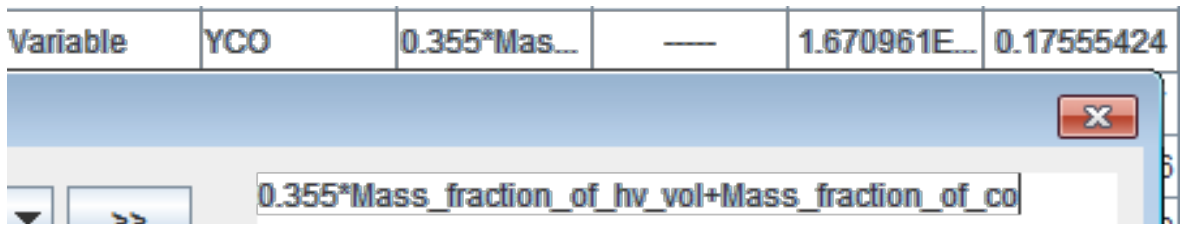

Figure 15. User-defined CO mass fraction variable $\mathrm{YCO}$ and its definition within ENERGICO.

In addition to mass-fraction mapping, it is also necessary to map the production rate of volatile gas species from the DPM source of the pseudo-volatile species in the CFD solution to the source terms of the user defined variables (e.g., "YCO"). For example, the $\mathrm{CO}$ mass production rate, WDOTCO, can be computed from the DPM volatile mass source and the DPM CO mass source (due to possible coal burnout) in the CFD solution as given in Figure 16.

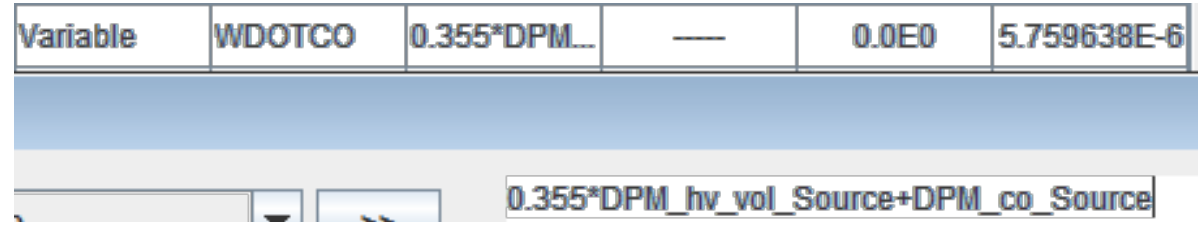

Figure 16. Definition of CO production rate from devolatilization and combustion of coal particles in ENERGICO.

To achieve effective zone partitioning for a coal gasifier/combustor, a variable that can be used to identify the extent of interaction between the discrete phase and the gas-phase, i.e., devolatilization and burnout, is very important. The basic ERN-extraction algorithm that we developed employs a total DPM mass-source variable to indicate regions where coal particles are active. This total DPM mass source variable is also specified as a user-defined function and is defined as

DPM mass source = DPM Burnout Rate + DPM Evaporation/Devolatilization Rate as shown in Figure 17.

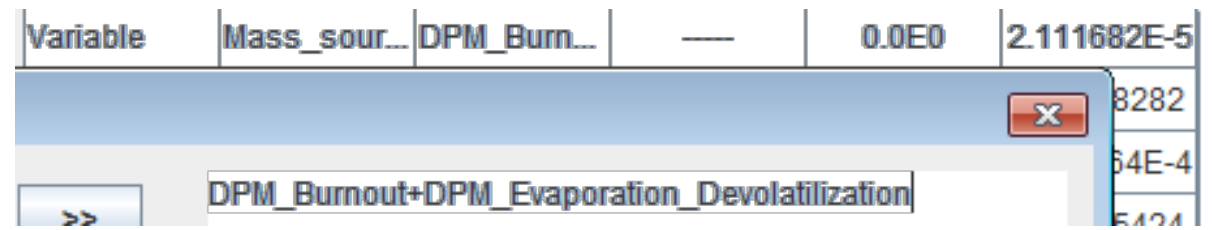

Figure 17. Definition of the total DPM mass source variable in ENERGICO.

A table listing all user-defined variables employed by our initial ERN-extraction algorithm is shown in Figure 18. 


\begin{tabular}{|c|c|c|c|c|c|}
\hline \multicolumn{5}{|c|}{ E Define Custom Variables } & $x$ \\
\hline \multicolumn{6}{|c|}{ User Defined Variables \& Constants } \\
\hline Data Type & Symbol & Formula & Value & Min. & Max. \\
\hline Variable & Mass_sour... & DPM_Burn... & - & $0.0 \mathrm{E} 0$ & $2.111682 E-5$ \\
\hline Variable & $\mathrm{YC} 2 \mathrm{H} 4$ & $0.5675^{\star}$ Ma... & - & 3.994888E.. & 0.27038282 \\
\hline Variable & $\mathrm{YH} 2$ & $0.0008^{\star M a} \ldots$ & - & $5.63156 \mathrm{E}-28$ & $3.811564 \mathrm{E}-4$ \\
\hline Variable & YCO & $0.355^{\star}$ Mas... & - & $1.670961 \mathrm{E}$. & 0.17555424 \\
\hline Variable & $\mathrm{YHCN}$ & $0.0767 *$ Мa... & $\ldots$ & 5.399258E.. & 0.03654337 \\
\hline Variable & WDOTC2 4 & $0.5675^{\star} \mathrm{DP} \ldots$ & - & $0.0 \mathrm{E} 0$ & 9.207309E-6 \\
\hline Variable & WDOTH2 & $0.0008^{*} \mathrm{DP} \ldots$ & - & $0.0 \mathrm{E} 0$ & $1.297947 \mathrm{E}-8$ \\
\hline Variable & WDOTCO & $0.355^{\star}$ DPM $\ldots$ & - & $0.0 \mathrm{E} 0$ & $5.759638 \mathrm{E}-6$ \\
\hline Variable & WDOTHCN & $0.0767^{\star} \mathrm{DP} \ldots$ & - & $0.0 \mathrm{E} 0$ & $1.244406 \mathrm{E}-6$ \\
\hline
\end{tabular}

Figure 18. A table showing all user-defined variables used by the basic ERN-extraction algorithm for coal gasification and combustion.

\section{Mapping Gas-Phase Species Variables}

When a CFD solution is imported, ENERGICO automatically maps known CFD variables such as pressure and temperature to their counterparts in the CHEMKIN-PRO reactor models. However, because user-defined variables are required to map the DPM variables from the CFD solution, the mapping between DPM variables and gas species must be done manually after all the user-defined variables are created. Figure 19 shows how DPM variables are linked to species in the gas-phase reaction mechanism through the user-defined variables. To ensure that the DPM mass sink terms work properly in the CHEMKIN-PRO reactor models, an "Include Sink Terms" option was introduced, as indicated by the red frame in Figure 19, and must be checked. Without this option, the negative species source values would be ignored. 


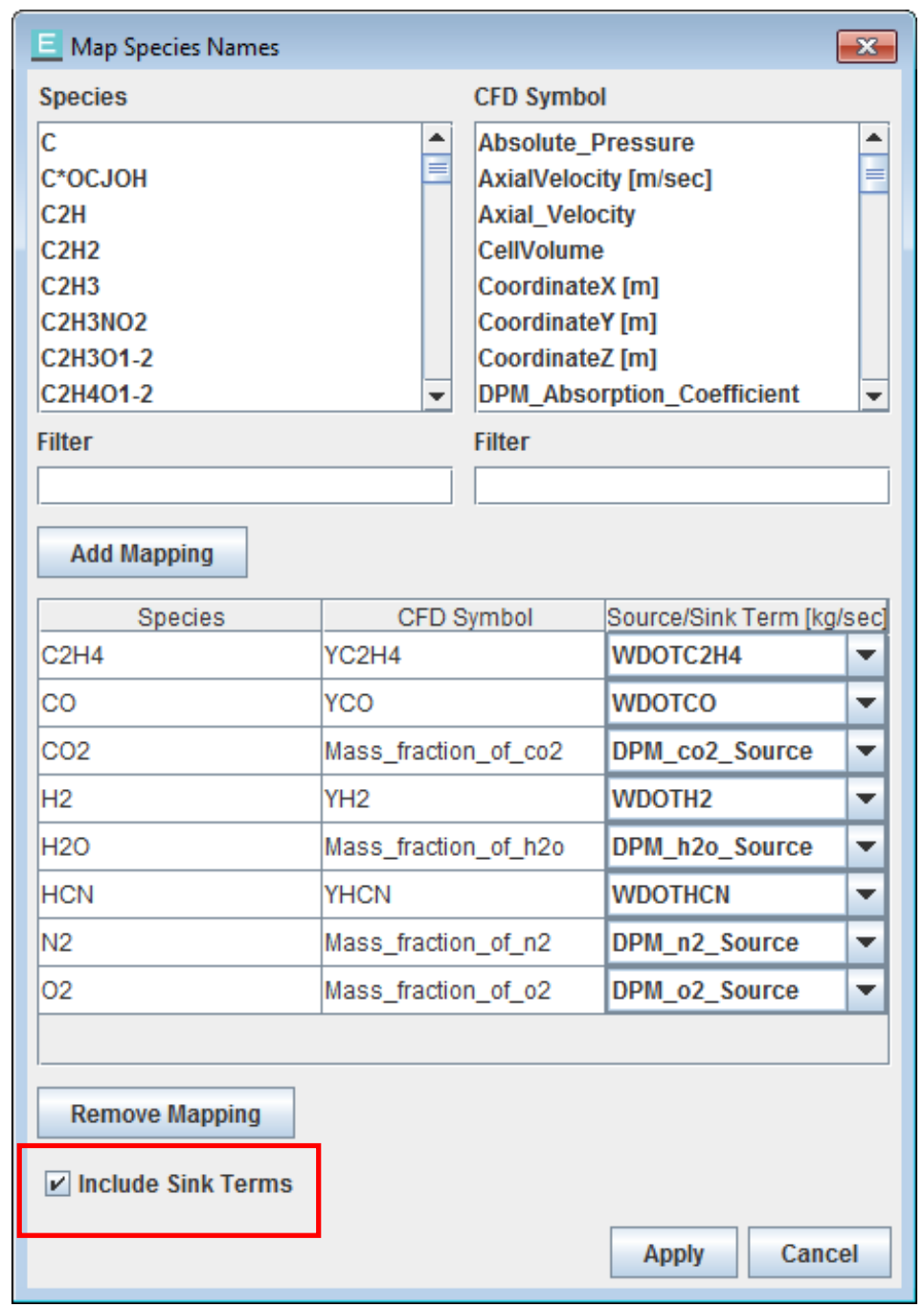

Figure 19. Mapping DPM variables in the CFD solution to mass fractions and mass production rates of gas species in CHEMKIN gas reaction mechanism.

\section{Mapping Other Variables}

All common CFD solution variables are recognized and mapped automatically to corresponding variable symbols used by ENERGICO. Any other solution variables that would be used to create ERN's for specific processes or applications have to be mapped manually. Although no mapping is necessary for pressure, it is important to verify the reference pressure. Double-checking all the units is also recommended. The "Map Variable" panel of ENERGICO is shown in Figure 20. 


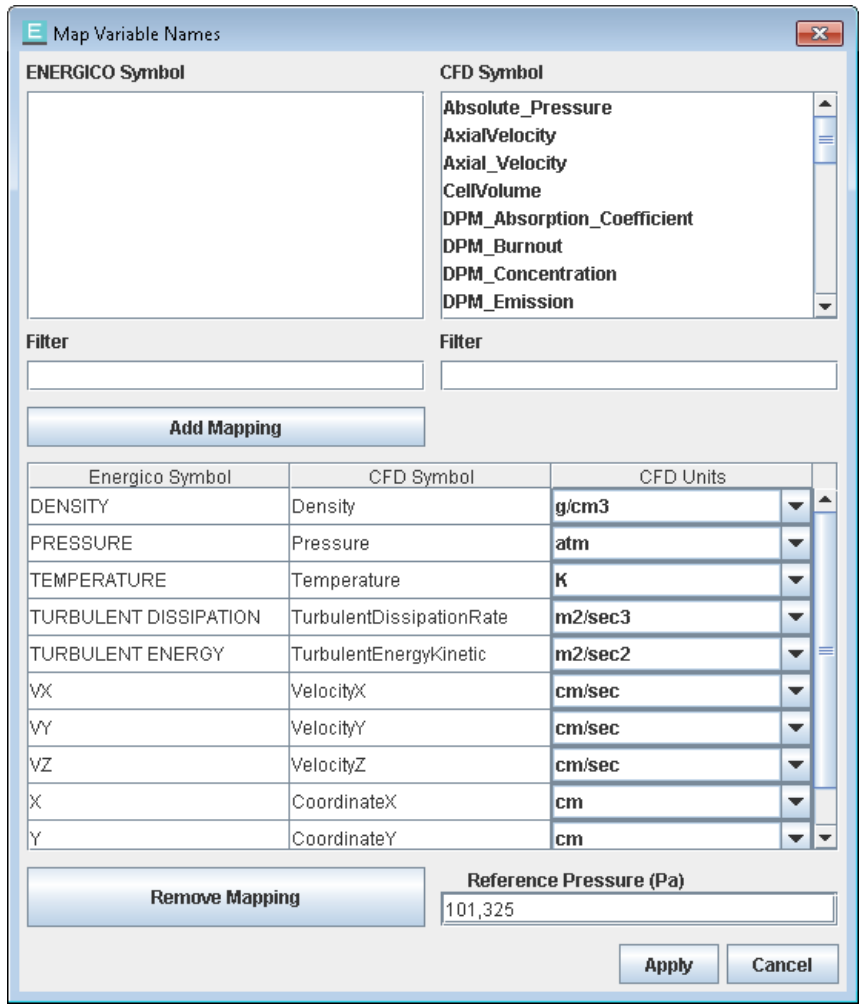

Figure 20. The ENERGICO "Map Variable" panel showing the mapping between CFD and ENERGICO symbols as well as their units.

\section{Creating Zoning Filters for a Coal Gasification Process}

Once all the variables/symbols are defined and mapped correctly, the workflow proceeds to the partitioning of the reactor volume and then to the creation of the ERN. After the reactor volume is divided into zones, ENERGICO will automatically determine the ERN structure and connectivity. Our initial ERN creation algorithm, described below, demonstrates the use of ENERGICO in partitioning a coal combustor/gasifier. This particular algorithm generates an ERN of modest size. It is always possible to use different approaches (variables and/or filters) to create ERN's of various sizes and complexities. Additional information about creating and applying filters can be found in the ENERGICO User Manual [2].

Each reactor within the ERN represents an actual region of the CFD flow domain. ENERGICO divides the flow domain into zones by using filters and through consideration of cell connectivity, such that the resulting zones are contiguous regions in the physical geometry. A filter is a logical criterion based on the value of a variable. By applying a sequence of carefully designed filters, we are able to capture important physical and chemical processes taking place in different zones of the flow domain. A good zone partitioning approach is critical to the accuracy of the ERN. It is important to keep variations of temperature and composition within a zone as small as possible.

The ERN-extraction algorithm we devised for a coal gasifier and combustor uses 6 filters. The filters are named after the variable employed in the filter criterion and they are listed below as well as in Figure 21, in the order of application. Some filters only apply to selected zones of the flow domain (i.e., based on previous filters). 


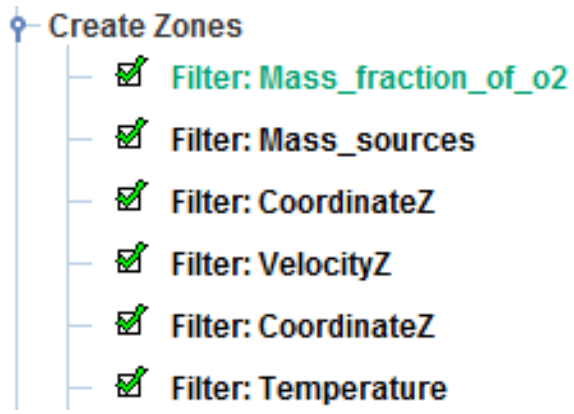

Figure 21. The sequence of filters employed by the ERN-extraction algorithm for coal gasifier and combustor.

The order and an explanation of each filter in the algorithm are as follows:

1. $\mathrm{An}_{2}$ mass fraction filter is first used to separate the air inlet region from the rest of the flow domain. No chemical activity is expected in the air inlet. This is depicted in Figure 22.

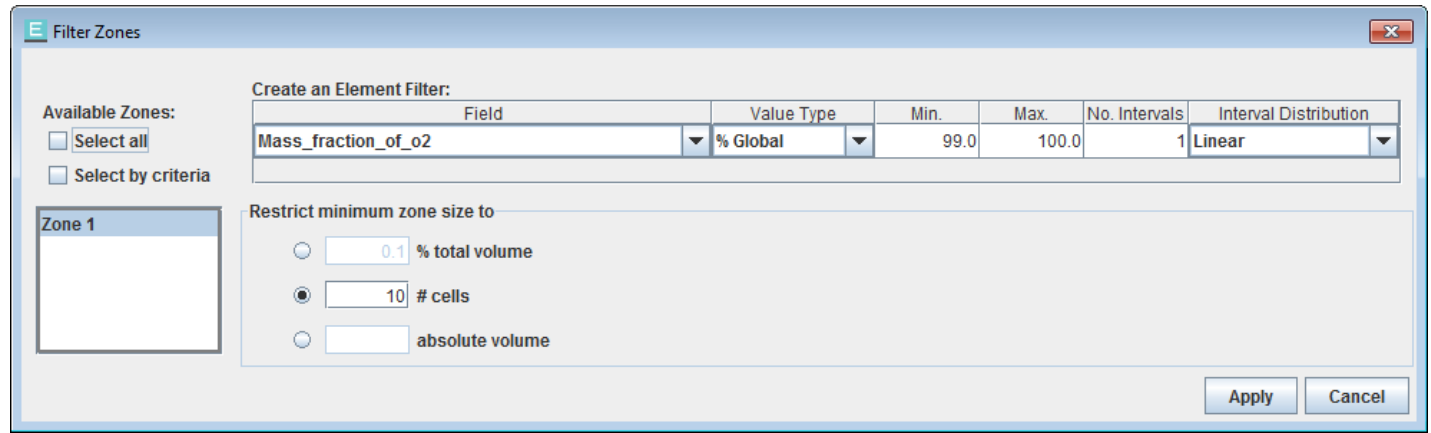

Figure 22. Applying an $\mathrm{O}_{2}$ filter in ENERGICO as part of the ERN partitioning.

2. A DPM mass-source filter is then applied to isolate regions with active coal devolatilization and combustion and to divide these devolatilization and combustion regions into smaller zones according to the local devolatilization rates. This is depicted in Figure 23.

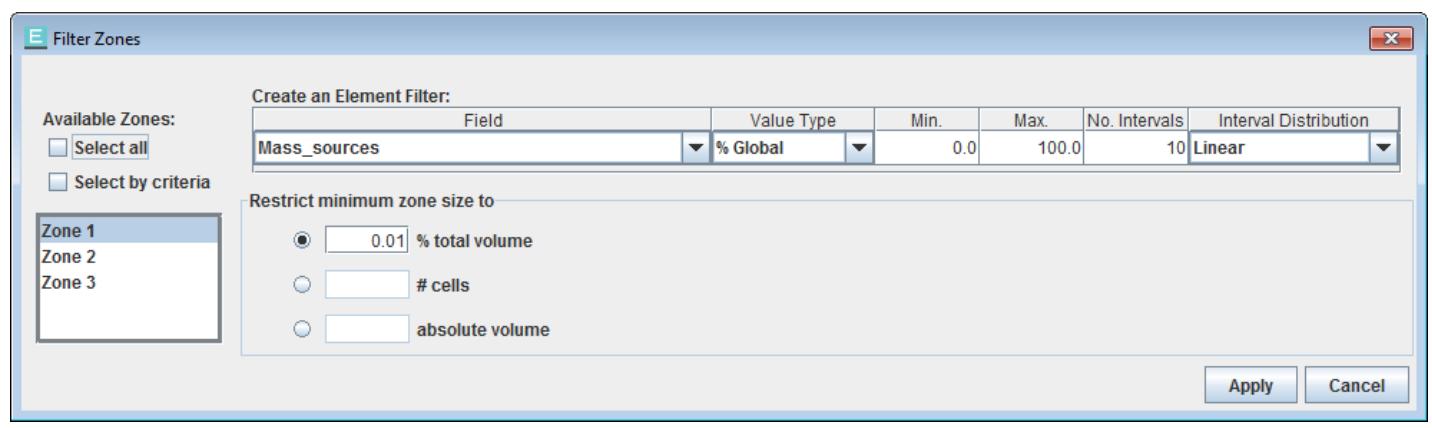

Figure 23. Applying a DPM filter in ENERGICO as part of the ERN partitioning.

3. A Z-coordinate filter (i.e., along the flow direction) is used to separate the coal feeding inlets from the main reactor body, as shown in Figure 24. We expect only negligible coal devolatilization will occur in the coal feed streams due to the relatively low temperatures there. 


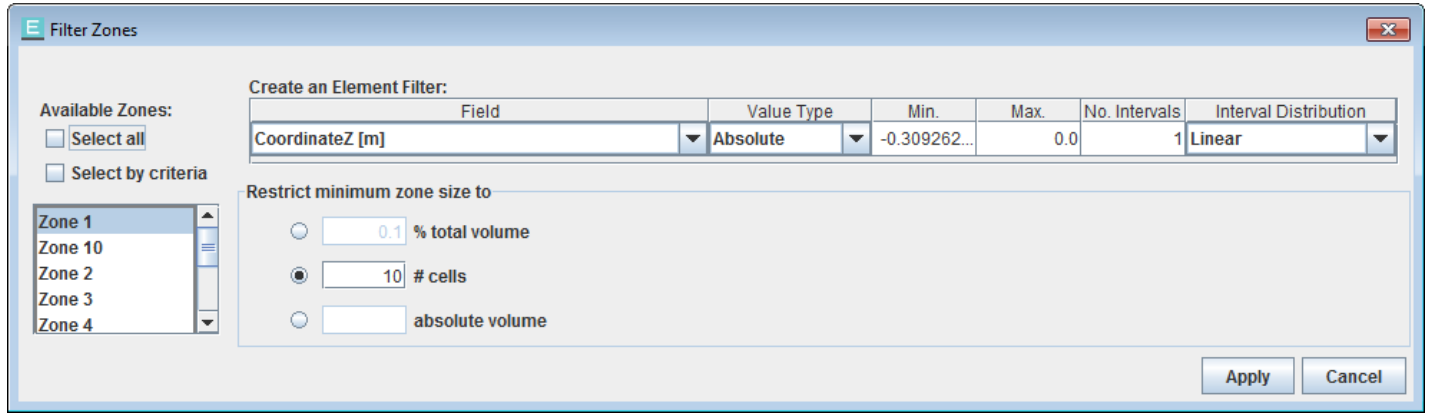

Figure 24. Applying a Z-coordinate filter in ENERGICO as part of the ERN partitioning.

4. An axial velocity filter is applied to zones in the main reactor body to isolate any recirculation regions, as shown in Figure 25. Back-mixing of hot combustion products provides a mechanism to stabilize flames or to sustain the reaction zone. It is important to identify these recirculation zones so that the stabilization mechanism can be preserved in the ERN.

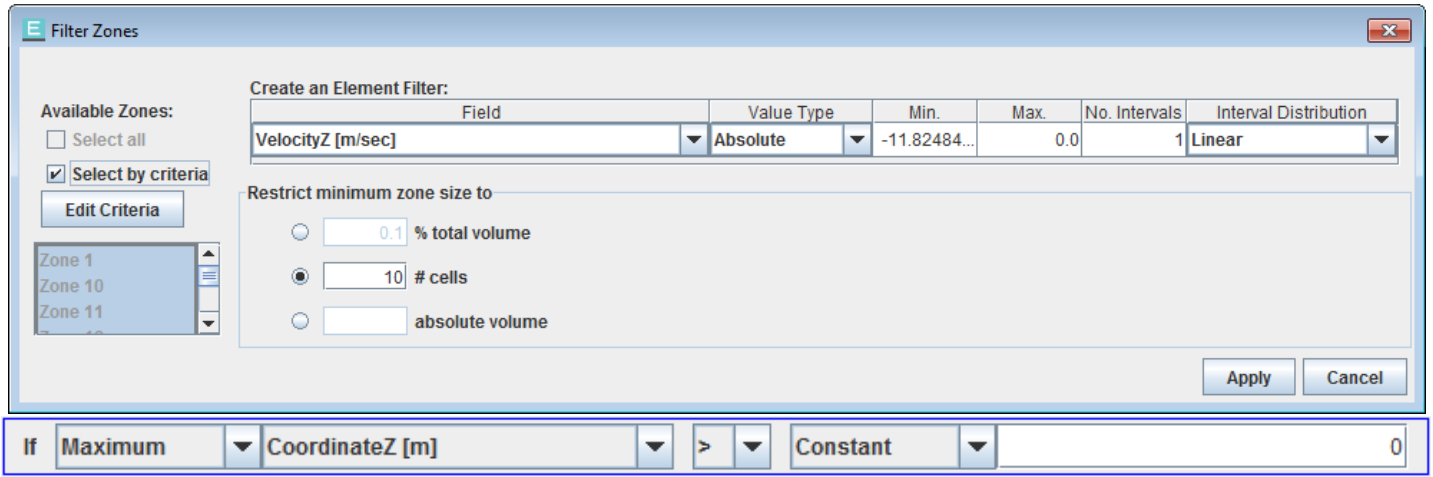

Figure 25. Applying an axial-velocity filter in ENERGICO as part of the ERN partitioning.

5. A second Z-coordinate filter is applied to the post-flame region, as shown in Figure 26. The post-flame region in a combustor resembles a plug-flow reactor. By dividing the post-flame region into small zones in the flow direction, the plug flow characteristics can be simulated using a series of PSRs, while still allowing entrainment along the flow direction.

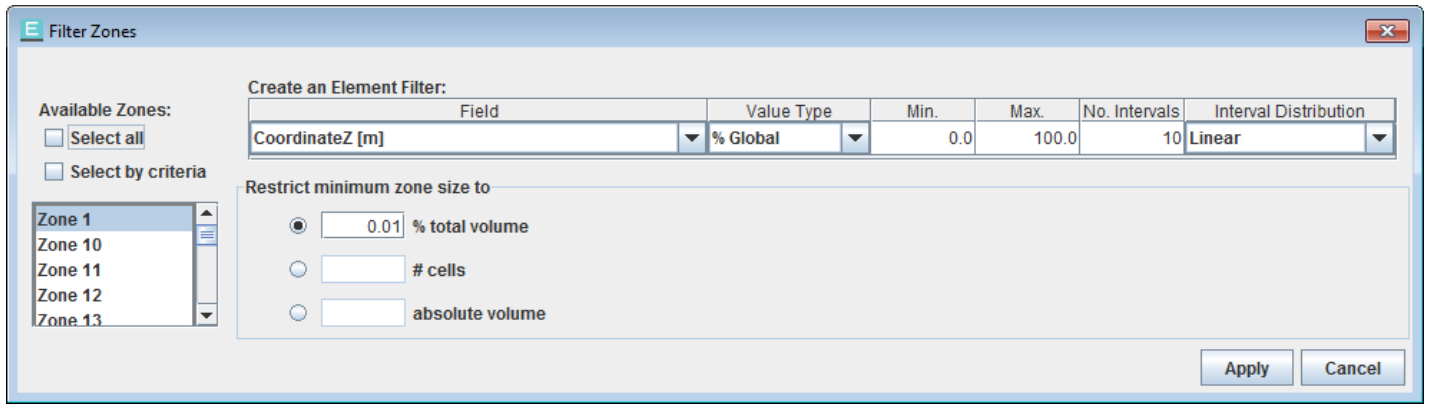

Figure 26. Applying a second Z-coordinate filter in ENERGICO as part of the ERN partitioning.

6. Finally, a temperature filter is applied to refine all zones in the gasifier/combustor, as shown in Figure 27. Since gas-phase chemical reactivity is sensitive to temperature, keeping temperatures as uniform as possible within each zone can preserve key chemical processes. Non-uniform temperature intervals are implemented in this filter due to the nonlinear 
dependence of reaction rate and temperature. The User-Defined temperature intervals are provided in the second screenshot in Figure 27.

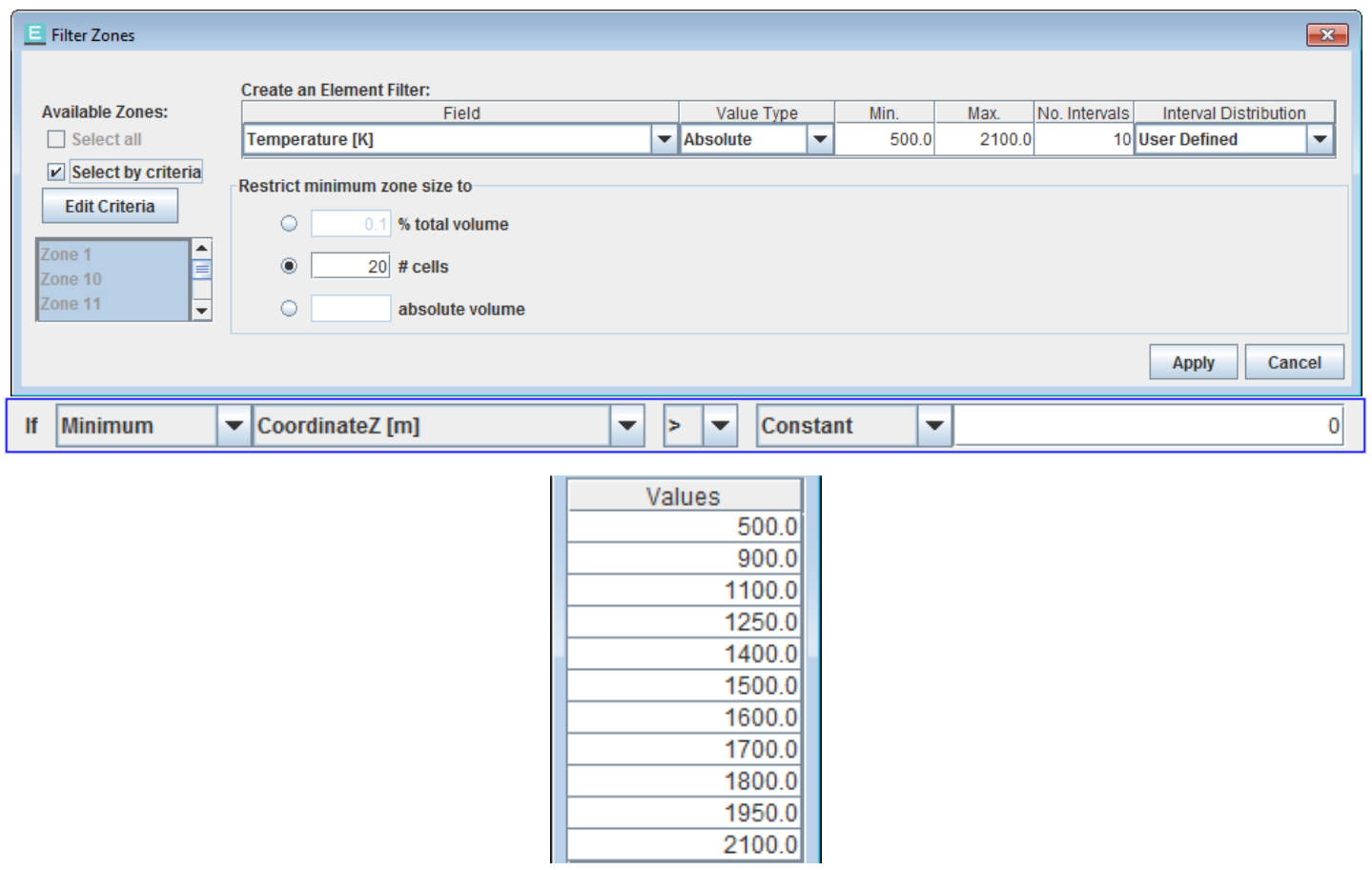

Figure 27. Applying a final temperature in ENERGICO as part of the ERN partitioning.

\subsubsection{Creating and Running the ERN}

For our sample gasifier case, a total of 91 zones were created from the filtering process described in Section 3.1.3, and they are shown in Figure 28. The ERN generated from this 91-zones of the coal combustor is shown in Figure 29 as it is displayed using the "ERN View" option in ENERGICO, which takes one to the CHEMKIN-PRO Interface. The two inlets, one for coal particle feed and the other for air, are modeled by two sets of Perfectly-Stirred Reactors (PSRs) in a series within the ERN. This is an indication that the flow along the inlet duct exhibits plug-flow-like characteristics. The plug-flow structure can also be found in the portion of ERN representing the downstream post-combustion region. The majority of the PSRs (or zones) and the complex network connectivity due to back-mixing are concentrated in the upstream half of the combustor where coal devolatilization and oxidation are most significant. 


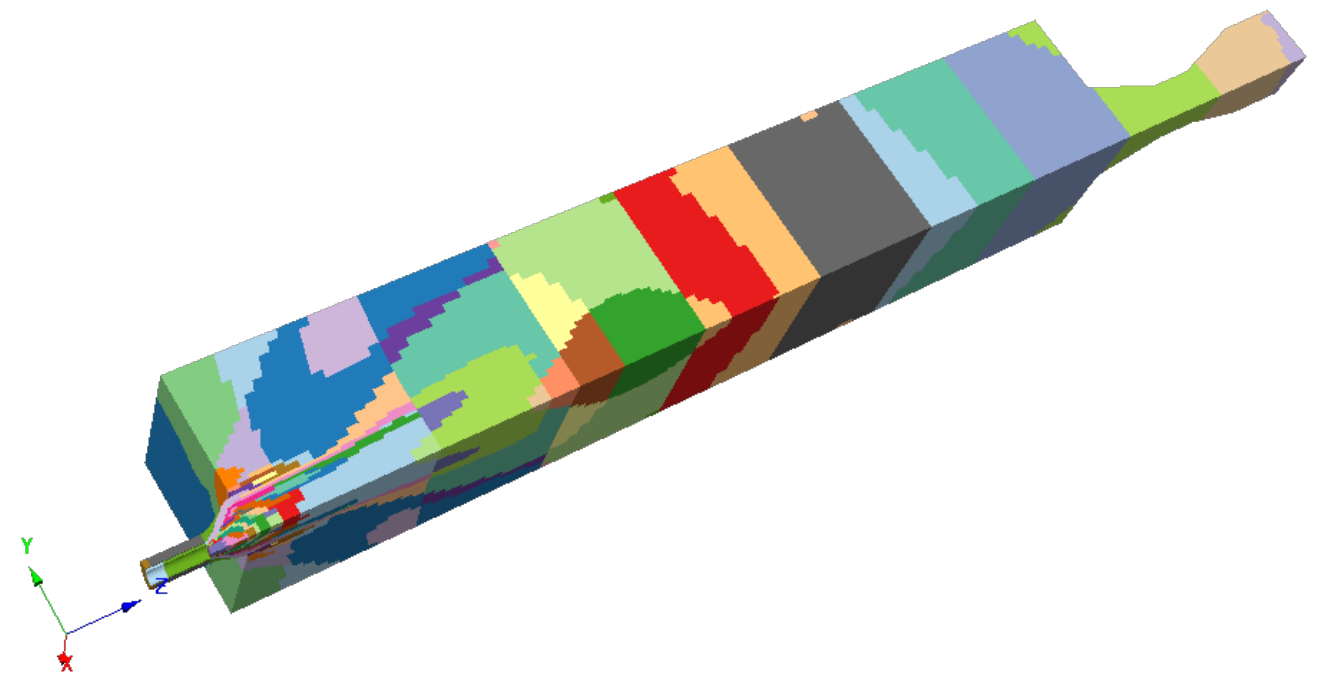

Figure 28. Zone partitions created by the basic ERN-extraction algorithm for the coal combustor.

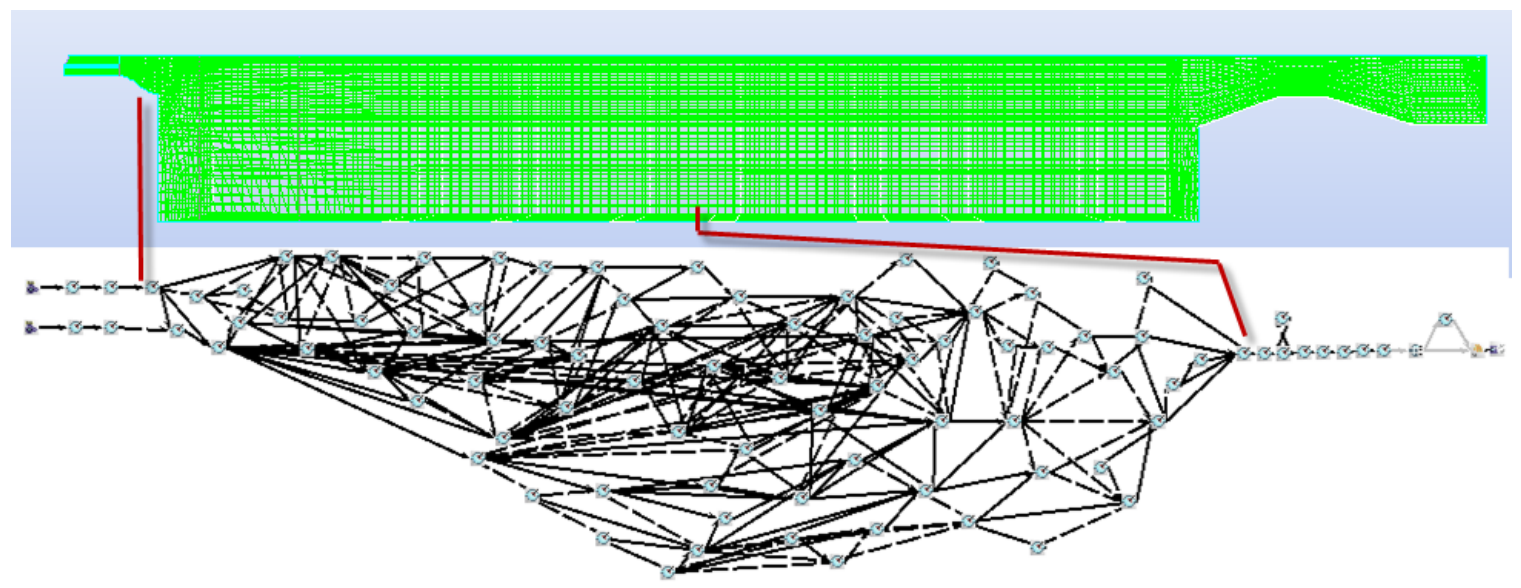

Figure 29. A side by side view illustrating the complexity of the ERN and relative locations of the reactors.

\subsubsection{Validating the ERN through Comparison to the CFD}

Once the ERN has been run within the ENERGICO/CHEMKIN-PRO framework, we can map the solution from the results back onto the CFD mesh for display within the ENERGICO interface. This allows verification of the ability of the ERN to represent the CFD model, by comparing the CFD solution to the ERN solution in the same geometric views. A detailed comparison and validation is discussed in Section 4.2, for example. We can also see at this stage in the workflow that the ERN may predict quantities and information that is not available in the CFD model. For example, the chemistry model used in the ERN contained a detailed $\mathrm{NO}_{x}$ submechanism, which allows us to see where $\mathrm{NO}_{\mathrm{x}}$ will be formed. This is shown for this sample problem in Figure 30. 


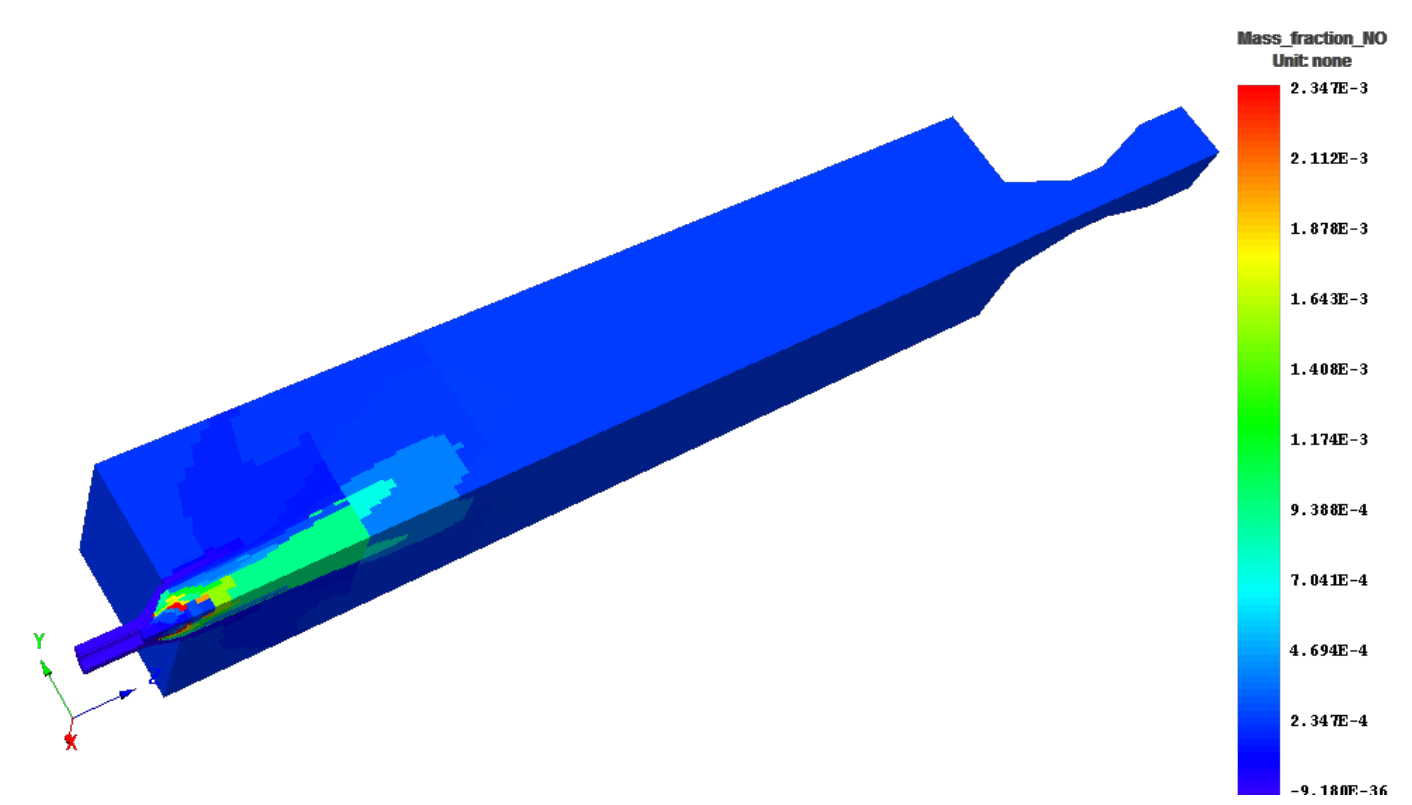

Figure 30. NO zonal results obtained by the ERN model.

\subsubsection{Exporting the ERN Model for Use with the CAPE-OPEN Interface}

The next step in the workflow is the export of the ERN model definition to a "CKCAPE" file. This is accomplished in the post-processing options within the ENERGICO/CHEMKIN-PRO interface. The CKCAPE file is a new file format that we created in order to export the ERN from CHEMKIN-PRO for use as a CAPE-OPEN Unit Operation. In other words, the CKCAPE file defines the specific ERN for this gasifier in a way that will allow the same gasifier ROM to be instantiated through the CO interface as a Unit Operation in the process flowsheet simulator.

\subsubsection{Running the CHEMKIN-CO ERN within Aspen Plus}

The steps to execute the ERN ROM within Aspen Plus (or any other CO-compliant process flowsheet simulator) are as follows:

1. From Aspen Plus, place Reaction Design's ERN Unit Operation (which is an encapsulated CO UO for COM) from the Aspen Plus Model Library, onto the flowsheet ;

2. When prompted, browse to and select the ERN CKCAPE file and load the ERN definitions, including the detailed mechanism to be used and the species for lumping;

3. Connect the UO inlet and outlet ports to appropriate Aspen Plus streams;

4. Solve the steady-state flowsheet in the Aspen Plus interface.

This workflow is analogous to that of APECS [3]. The approach is also compatible with any other CAPEOPEN-compliant simulator, such as COCO [5]. An example of an ERN-UO displayed within the Aspen Plus interface after loading is shown in Figure 33. 


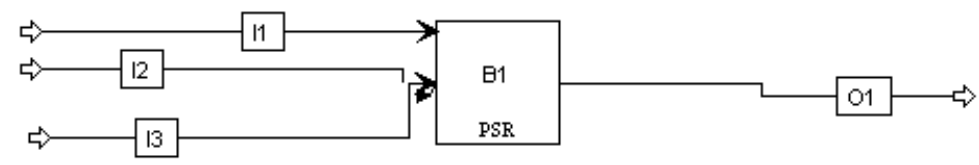

Figure 31. An Aspen Plus flowsheet containing the PSR Cluster.

The result of the ROM ERN solution can be obtained in the usual way from the Aspen Plus graphical user interface. In addition, detailed information about species that are not tracked in the Aspen Plus simulation are available in the UO report. In general the ERN output is the state of the gas (including temperature, pressure, and species composition) at the ERN outlet, which corresponds to the physical gasifier's outlet flow.

\subsection{Implementation of the CAPE-OPEN Interface for ERN Models}

In the CHEMKIN-PRO software framework, each reactor model exists in the form of a program that reads input files and writes the results to output files, from within a general-purpose standalone user interface. The user interface writes the input files, invokes the simulation programs, and reads the solution files for analysis and visualization. One objective of this project was to re-package the detailedkinetics Perfectly Stirred Reactor (PSR) model and the more general ERN, to make them available for process simulation as CAPE-OPEN compliant Unit Operations. This required a replacement of the input/output structure to allow for efficient and frequent calling of the $\mathrm{CO} \mathrm{UO}$ during an iterative flowsheet calculation.

We set out to make the CHEMKIN-based ERN models available as CAPE-OPEN compliant Unit Operations using Microsoft's Component Object Model (COM), because this is the middleware used for all CAPE-OPEN Unit Operations currently.

The CO UO interface specification is well documented by Co-LaN [5]. At the start of the project, we used VB6 as the underlying programming language to implement the CO UO COM interface, due to the prevalence of experience with VB6 in the CO community and with Aspen Plus support. The conversion of our ERN model architecture to CO UO compliant COM DLLs involved several tasks:

(1) The conversion of a standalone Mechanism Pre-processing executable, for parsing reaction mechanism files prior to simulation using the ERN models, into an DLL callable by VB6;

(2) The conversion of the existing standalone PSR simulation executable (written in $\mathrm{C}++$ and Fortran) into a DLL with an API that is suitable for support of the CO UO standard, instead of being file I/O-dependent for definition of the reactor specification and inlet conditions;

(3) Development of a VB6 intermediary software component that interacted with the PSR C++ API on one end and with the COM CAPE-OPEN API on the other end;

(4) Re-implementation of the tear-stream algorithm that is used in CHEMKIN-PRO so that it can be encapsulated within the $\mathrm{CO} \mathrm{UO}$, to allow the complex ERNs to operate outside of the CHEMKIN-PRO user interface.

In CHEMKIN-PRO simulations, the PSR simulation executable within an ERN reads several files for obtaining information required for the simulation. One or more of the files contain a list of chemical species involved in the detailed chemical mechanism, the thermodynamic data for all species included in reactions and the reaction strings, stoichiometric coefficients, as well as the temperature-dependent 
reaction rates for all reactions. These files are collectively called the mechanism files. In addition, the program reads a control file which, among other things, sets operating conditions for the reactor such as pressure and volume, as well as inlet conditions such as temperature, mass flow rate, and molar compositions of the inlet stream. In the context of the UO, a user should be able to select a mechanism (or "chemistry set") and supply the data files associated with that mechanism; whereas the information represented by the control file should come from inlets to the PSR and as parameters for the UO. With this view, we have replaced the contents of the control file with a data structure and replaced the reading of a control file with a direct call to the PSR containing the data structure as an argument. Thus we have converted the PSR simulator engine from a main executable to a DLL that presents a callable interface to other executable programs. Previously existing uses of the simulator were retained by providing a thin executable main program that invokes the DLL interface.

The PSR simulation program writes two kinds of output files. The solution output file contains the final conditions, such as reactor temperature, outlet flow rate, and the outlet composition of all species involved in the mechanism. The diagnostic output file contains informative messages indicating the progress of the calculations and any errors that occurred. For the purpose of using the simulator as a flowsheet UO, we have suppressed the writing of the solution file, instead providing callable interfaces in the DLL for obtaining the final solution data. The diagnostic file is still generated for troubleshooting purposes.

The CAPE-OPEN UO API specifies standardized calling interfaces for obtaining information from inlet ports and sending information to outlet ports. Every UO must supply a DLL that performs the operation represented by the UO and presents a CAPE-OPEN conforming interface for the inlet and outlet ports. We have developed a software component called a driver to serve this purpose. On the one hand, the PSR driver interacts with the user interface in the flow diagram through CAPE-OPEN calls. On the other hand, it interacts with the underlying simulator engine through the call interface presented by the PSR or ERN DLL.

For the purposes of testing our CO implementation, we decided to use the COFE simulator [27] as well as Aspen Plus. The main reason for this was concern over some extensions to the CO interface that had been introduced into Aspen Plus. To avoid inadvertently taking advantage of these extensions, we did not want to rely solely on Aspen Plus to determine the CO UO compliance, even though it was the target CO simulation environment for this work.

In the interface between the CHEMKIN ERN CO UO DLL and COFE or Aspen Plus, we recognized that the species names used by the process flowsheet streams may not be the same as the symbols used in the mechanism files employed by the ERN simulator. Thus we had to assure a one-to-one correspondence map between the two sets of names. To address this, we designed a Graphical User Interface (GUI) component to present the two lists to the user and let the user map the corresponding species names, with automatic mapping based on string matching where possible. While the full implementation of this UI was not possible during the scope and timeline of this project, a temporary capability to read the mapping information from a file was implemented in the interim.

\subsubsection{Lumping Method Implementation to Assure Mass and Element Conservation}

One of the advantages of utilizing detail-kinetics equivalent reactor networks (ERNs) in coal gasifier simulation is the ability to predict process by-products and emissions accurately. However, when the ERN model is incorporated into a flowsheet simulator, many species in the detail kinetics mechanism do not have unique counterparts in the short species list tracked and solved for by the flowsheet simulator. 
This species mismatch would create mass (or flow rate) and chemical-element imbalances without an appropriate measure to redistribute the mass and elements of those extra species.

We evaluated several methods, including equilibrium calculation, for redistributing mass and elements between two sets of species. The challenge was to conserve the total free energy, the mass, and the chemical elements, while maintaining accurate concentrations of key major species, such as $\mathrm{H}_{2}$ and $\mathrm{CO}$. We went through a number of iterations on the methodology and finalized on a plan in which mass from trace species is redistributed by spreading elements of the trace species to only a few "target" species that are explicitly tracked by the flowsheet simulator. The target species are selected according to their elemental composition, mass fraction, and the accuracy demanded for those species. The number of target species must be equal to the number of elements comprising all the extra (trace) species; in addition, their mass fractions should be relatively large, relative to the trace species mass. Once the target species are chosen, the total mass/mole of each element in the extra species can be computed. The massfraction change of a target species, $\Delta Y_{k}$ is then determined by solving a set of algebraic equations derived by chemical-element conservation:

$$
\sum_{k=1}^{N_{\text {target }}} \frac{\Delta Y_{k} \sigma_{m, k}}{M W_{k}}=M_{m} .
$$

Here $\sigma_{m, k}$ is the number of elements $m$ in target species $k, M W_{k}$ is the molecular weight of the target species $k$, and $M_{m}$ is the number of moles of element $m$ in the sum of all extra species, as follows:

$$
M_{m}=\sum_{l=1}^{N_{\text {exra }}} \frac{Y_{l} \sigma_{m, l}}{M W_{l}} .
$$

Because the number of target species equals the number of elements in the extra species, the mass fraction adjustments, $\Delta Y_{k}$, can be uniquely determined from Equation (1). After $\Delta Y_{k}$ are obtained, the new mass fractions of the target species can be computed initially as

$$
Y_{k}^{\prime}=Y_{k}+\Delta Y_{k}
$$

and then normalized by

$$
Y_{k}^{\prime \prime}=\frac{Y_{k}^{\prime}}{\sum_{j} Y_{j}^{\prime}}\left(\sum_{l=1}^{N_{\text {extra }}} Y_{l}+\sum_{j=1}^{N_{\text {target }}} Y_{j}\right)
$$

to assure summation to unity over all the major (COSE-tracked) species. The conservation of mixture Gibb's free energy is also imposed by iteratively solving a new gas temperature $T^{\prime \prime}$ from

$$
H\left(T^{\prime \prime}, Y^{\prime \prime}\right)-T^{\prime \prime} S\left(T^{\prime \prime}, Y^{\prime \prime}\right)=H(T, Y)-T S(T, Y)
$$

Since all extra species are expected to exist in trace quantities, the impact of mass redistribution on target species concentrations and on the gas temperature should be very small.

The extra species redistribution method outlined here was added to our CAPE-OPEN ERN module and then verified for specific gasifier ERN examples. The final validation test and results are discussed in Section 4.3. 


\subsection{Development of a New Coal Kinetics Module}

In addition to enabling ERN-based ROMs within CAPE-OPEN compliant process flowsheet simulators, the other major focus of this project was the evaluation of chemical kinetics representations for coal gasification. The simplest approach for the ROM chemistry was to use the devolatilization source terms directly from the CFD model and then only enhance the gas-phase kinetics for the pyrolysis, combustion and emissions-production chemistry. This approach was used as the baseline approach and demonstrated to be effective for two different coal gasifier models. These results are discussed in more detail in Section 4.

In addition to this baseline, however, we were interested in evaluating methods to bring more physics and more detail into the gasification process representation, in order to provide a more predictive simulation capability. Towards this end, we undertook a detailed literature survey on coal gasification modeling. This revealed two major approaches for modeling the coal gasification process : the empirical functional groups (FG) $[28,29]$ and the Chemical Percolation Devolatilization (CPD) model [30].

A key issue to both models is the need for additional coal-structure parameters, such as initial concentrations of each functional group, coordinate number, and initial fraction of intact links. These additional coal parameters can only be determined by employing advanced experimental techniques, such as carbon-13 nuclear magnetic resonance $\left({ }^{13} \mathrm{C}\right.$ NMR) spectroscopy; such data are only available for a limited number of research and commercial coals. For coals with no available structural information, various methods have been developed to correlate the structural parameters needed by the devolatilization models to the coal elemental composition, which can be obtained via ultimate analysis of the coal. Zhao, et al. [31] used an interpolation method to obtained structural information from a set of reference coals for which the elemental composition and necessary structural parameters are known experimentally. Genetti and Fletcher [32], using a different set of reference coals, established empirical formulations that correlate coal structural parameters to the coal's elemental composition. Both approaches yield reasonable predictions for coals that lie within each correlation's applicable range. For our devolatilization models, we decided to follow the interpolation approach by Zhao et al., because the coal library can be expanded to improve accuracy and coverage for a wider range of coal ranks. The empirical polynomials obtained by Genetti and Fletcher required re-fitting whenever changes were made to the reference coals.

We next collected the information required to build the coal library and developed an interpolation algorithm that would be effective for an unstructured data set with two or more independent parameters. These components became part of what became the CHEMKIN Coal Kinetics Module. Our goal was to facilitate both the empirical functional-group method as well as the more detailed CPD modeling for coal devolatilization kinetics.

The CHEMKIN Coal Kinetics Module is a general module that facilitates quantitative predictions of coal particle size evolution, tar yield, tar molecular-weight distribution, and yields of individual gas species from a coal gasification process. There are three main components in the Coal Kinetics Model:

1. An interactive interface that guides users through the process that starts with entering necessary coal analysis data and ends with generating a template Surface CHEMKIN coal devolatilization mechanism appropriate for that data, where the resulting mechanism can be modified by users later if necessary;

2. A software module that computes coal particle size distribution, tar product rates and individual gas-species production rates, during a reacting-flow simulation (e.g., a PSR or ERN model) for a given surface mechanism. 
The "template" surface mechanism is tailored to the particular coal defined by characteristic data provided by users in addition to kinetic parameters for bridge breakup, cross-linking, and light gas release common to all coal ranks. The generic reaction pathways for coal devolatilization and oxidation that are initially provided in the template mechanism could be further improved upon by adding more reaction paths and/or by adjusting the rate parameters. This flexibility is provided to allow tuning to experimental data as necessary and also to allow researchers to explore new ideas for the mechanism representation. The software module is a collection of chemical kinetic utilities based on proven coal models such as the Chemical Percolation Devolatilization (CPD) [33] and Functional Group (FG) [34] models. The software module is designed to interface with any CHEMKIN-PRO reactor/flame model that also enable particle tracking. The module is designed to be fully expandable for adoption of new advancements in coal modeling in the future.

The Coal Kinetics Module is built upon the existing functionalities of CHEMKIN and the Particle Tracking module, which are described in more detail in the CHEMKIN-PRO user manuals [14]. New devolatilization-related kinetics features are integrated into the surface-chemistry handling within CHEMKIN. The evolution of coal particle size distribution is resolved by the Particle Tracking module using either sectional or moments method. Moreover, concentrations of bulk species comprising the coal are tracked by the Particle Tracking module, so that mass and element conservations are strictly enforced. The Coal Kinetics Module itself will include a coal property library and some percolation theory utilities. The coal property library contains properties of well-characterized coal samples and is used to interpolate properties of less-known coals based on their elemental compositions [31, 32]. The percolation math routines support the calculation of coal lattice fragmentation rates for tar yield and tar mass distribution [33].

The CHEMKIN Coal Kinetics Model, is designed to be a generalized version of the Chemical Percolation Devolatilization (CPD) model, which was originally developed by Fletcher and co-workers [33]. It is adapted to take advantage of the surface-chemistry capabilities available in CHEMKIN and to provide extensibility and flexibility for the user.

To adapt the CPD model to CHEMKIN, the conceptual coal structure components are converted into surface and bulk species in the Coal Kinetics Model. Processes controlling evolution of tar and light gases during coal pyrolysis are compiled into a surface reaction mechanism. The benefits of making this CHEMKIN adaptation are:

1. Key steps of coal devolatilization become readable and editable since the surface mechanism is a text file;

2. Kinetic constants for individual pyrolysis reactions can be easily modified so that a parameter study can be performed to optimize the model;

3. Surface chemistry compatibilities already in CHEMKIN make sensitivity analysis available to identify critical path under various operating conditions;

4. The coal devolatilization model becomes extensible by any user;

5. The model is readily integrated with detailed gas-phase reaction kinetics (CHEMKIN), as well as particle/aerosol models in the Particle Tracking module.

The Coal Kinetics Model can predict the compositions and the amount of light gases released from coals. In combination with the CHEMKIN Particle Tracking module, the models can even provide coal/char particle size evolution during the devolatilization process. The weight distribution of tar species in gas phase can be constructed from concentrations of a species group of gas species of which production rates are derived from the percolation model. 


\subsubsection{Surface Chemistry Representation of Devolatilization}

The Coal Kinetics Module employs percolation lattice statistics to describe the generation of tar precursors during coal pyrolysis. It represents the macroscopic structure of unreacted coal as an infinite Bethe pseudo lattice or tree. The fused aromatic-ring clusters of various sizes are nodes/sites of the lattice. The heteroatom molecules interconnecting the sites are interpreted as bridges. The sites can also be bonded by char links. Bridges are key reaction centers during pyrolysis. A labile bridge can either dissociate or condense instantaneously into a char link. Broken bridges and peripheral molecules attached to the sites are treated as side-chains by the module. Figure 32 gives a simple example illustrating the relationship between the coal molecular structures and the infinite lattice model. Detailed descriptions of this infinite lattice interpretation of coal structure can be found in the original reports of the CPD model [33], the FG-DVC model [34], and the FLASHCHAIN model [35, 36].

Chemically, the Coal Kinetics Module considers coals as solid surrogates composed of fused aromaticring clusters, labile bridges, char links, and side-chains. Since a broken bridge and side-chain are indistinguishable statistically, a side-chain is modeled as one half of a labile bridge. Furthermore, the labile bridge (and hence the side-chain) is assumed to only consist of functional groups. A functional group is a specific group of atoms within the coal molecule that characterizes the release of a particular light gas.

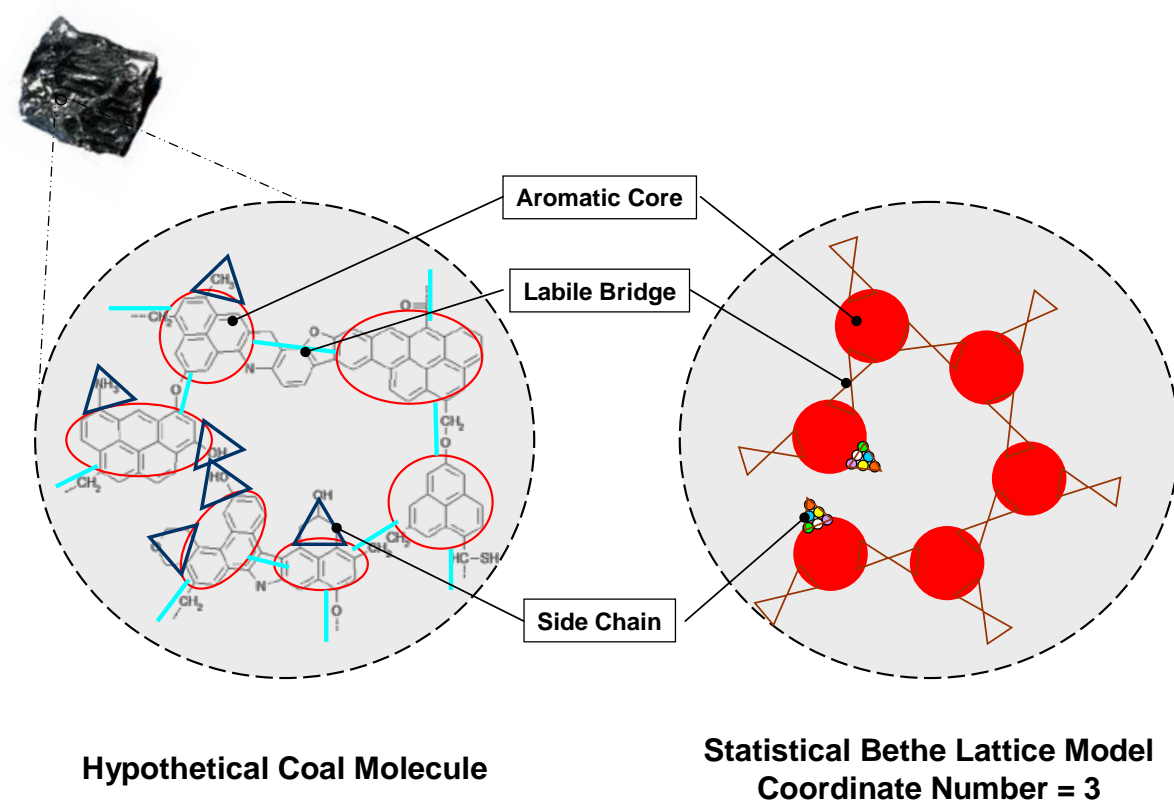

Figure 32. A graphical presentation of coal lattice model.

In the Coal Kinetics Module, the functional group components are identified by their corresponding gas products and by the nature of the bond breaking [37]. The thermal cracking of functional-group species from coal is described by a first-order reaction [37]. The kinetic rates are derived from experiments [37-39] and are found to be relatively insensitive to coal ranks [38]. Figure 33 lists typical coal functional group components and their corresponding kinetics-rate expressions [40]. The formation of light gases is not solely from direct cracking of functional-group species; light gases can be released during the generation of tar precursors as well [37,40]. The parallel independent paths for light gas evolution can be well captured by the Coal Kinetics Module, because both labile bridge and side-chain are solid mixtures of functional group species. The functional group components in side-chains represent the contribution of 
distinct gas-phase species through thermal cracking of coal molecules, while those residing in labile bridges mimic the parallel gas evolution path that is coupled to tar formation. Figure 34 provides a list of all the Coal Kinetics Module components, along with their definitions.

\begin{tabular}{|c|c|c|c|}
\hline & $\begin{array}{c}\text { Composition } \\
\text { parameter }\end{array}$ & $\begin{array}{c}\text { Gillette } \\
(\mathrm{c} 5 \mathrm{~b}) \\
(\mathrm{Wt} \% \mathrm{DAF})\end{array}$ & $\begin{array}{l}\text { Kinetic rate constants } \\
\text { (L6c) } \\
\left(\mathrm{s}^{-1}\right)\end{array}$ \\
\hline & $\mathrm{C}$ & 0.720 & \\
\hline & $\mathrm{H}$ & 0.047 & \\
\hline & $\mathrm{N}$ & 0.012 & \\
\hline & S(organic) & 0.005 & \\
\hline & $\mathrm{O}$ & 0.216 & \\
\hline & & 1.000 & \\
\hline$Y_{1}^{0}$ & $\mathrm{CO}_{2}$-extraloose & 0.0065 & $k_{1}=0.34 E+15 \exp [-(22,413 \pm 2000) / T]$ \\
\hline$Y_{2}^{1}$ & $\mathrm{CO}_{2}$-loose & 0.0652 & $k_{2}=0.48 E+15 \exp [-(29,591 \pm 3630) / T]$ \\
\hline$Y_{3}^{2}$ & $\mathrm{CO}_{2}$-tight & 0.0283 & $k_{3}=0.11 E+16 \exp [-(38,315 \pm 3630) / T]$ \\
\hline$Y_{4}^{0}$ & $\mathrm{H}_{2} \mathrm{O}$-loose & 0.0503 & $k_{4}=0.17 E+15 \exp [-(27,500 \pm 2300) / T]$ \\
\hline$Y_{5}^{0}$ & $\mathrm{H}_{2} \mathrm{O}$-tight & 0.0000 & $k_{9}=0.17 E+15 \exp [-(32,700 \pm 3300) / T]$ \\
\hline$Y_{6}^{0}$ & $\mathrm{CO}$-Ether-loose & 0.0350 & $k_{6}=0.17 E+12 \exp [-(25,000 \pm 2500) / T]$ \\
\hline$Y_{7}^{0}$ & $\mathrm{CO}$-Ether-tight & 0.1375 & $k_{7}=0.10 E+15 \exp [-(40,500 \pm 6000) / T]$ \\
\hline$Y_{8}^{0}$ & HCN-loose & 0.0071 & $k_{8}=0.17 E+13 \exp [-(30,000 \pm 1500) / T]$ \\
\hline$Y_{9}^{0}$ & HCN-tight & 0.0154 & $k_{9}=0.10 E+15 \exp [-(47,500 \pm 4750) / T]$ \\
\hline$Y_{10}^{0}$ & $\mathrm{NH}_{3}$ & 0.0000 & $k_{10}=0.12 E+13 \exp [-(27,300 \pm 3000) / T]$ \\
\hline$Y_{11}^{10}$ & $\mathrm{CH}_{x}$-aliphatic & 0.1251 & $k_{11}=0.17 E+15 \exp [-(30,000 \pm 1500) / T]$ \\
\hline$Y_{12}^{0}$ & Methane-extraloose & 0.0000 & $k_{12}=0.17 E+15 \exp [-(30,000 \pm 1500) / T]$ \\
\hline$Y_{13}^{12}$ & Methane-loose & 0.0160 & $k_{13}=0.15 E+14 \exp [-(30,000 \pm 2000) / T]$ \\
\hline$Y_{14}^{0}$ & Methane-tight & 0.0160 & $k_{14}=0.34 E+12 \exp [-(30,000 \pm 2000) / T]$ \\
\hline$Y_{15}^{10}$ & $\mathrm{H}$-aromatic & 0.0126 & $k_{15}=0.16 E+08 \exp [-(23,000 \pm 2300) / T]$ \\
\hline$Y_{15}^{0}$ & Methanol & 0.0000 & $k_{16}=0.00 E+00 \exp [-(30,000 \pm 0) / T]^{*}$ \\
\hline$Y_{17}^{0}$ & Aldehyde & 0.0000 & $k_{17}=0.00 E+00 \exp [-(30,000 \pm 0) / T]^{*}$ \\
\hline$Y_{18}^{0}$ & C-nonvolatile & 0.4800 & $k_{18}=0$ \\
\hline$Y_{19}^{0}$ & S-organic & 0.0050 & \\
\hline & Total & 1.0000 & \\
\hline$X_{0}$ & Tar & 0.2500 & $K_{T}=0.45 E+14 \exp [-(26,400 \pm 1500) / T]$ \\
\hline \multicolumn{4}{|c|}{ Cracking rates: } \\
\hline & Parafinin-oiefins & & $k_{\mathrm{OL}}=0.15 E+12 \exp [-(27,600) / T]^{*}$ \\
\hline & Olefin-acetylene & & $k_{\mathrm{AC}}=0.21 E+08 \exp [-(22,000) / T]^{*}$ \\
\hline
\end{tabular}

Figure 33. An example of functional group components and their kinetic rates [40]. 


\begin{tabular}{|c|c|c|}
\hline \multirow{2}{*}{\multicolumn{2}{|c|}{$\begin{array}{l}\text { Symbol Component } \\
\text { Aromatic Core }\end{array}$}} & Definition \\
\hline & & $\begin{array}{l}\text { Fused aromatic ring cluster that forms a site } \\
\text { of the Bethe lattice }\end{array}$ \\
\hline & Labile Bridge & $\begin{array}{l}\text { Molecular structures that serve as bridges } \\
\text { connecting aromatic cores/sites }\end{array}$ \\
\hline & Activated Bridge & $\begin{array}{l}\text { Labile bridge that is active and ready to } \\
\text { break down }\end{array}$ \\
\hline$\$$ & Side Chain & $\begin{array}{l}\text { Peripheral molecular structures attached to } \\
\text { aromatic cores }\end{array}$ \\
\hline $\begin{array}{l}\circ 00 \\
\circ \circ 0\end{array}$ & Functional Group & $\begin{array}{l}\text { Specific group of atoms within coal } \\
\text { molecules that characterize the release of } \\
\text { light gases }\end{array}$ \\
\hline & Char Link & Permanent bond that fuses aromatic cores \\
\hline
\end{tabular}

Figure 34. Definitions of the Coal Kinetics Module lattice model components.

One important objective of coal devolatilization modeling is the prediction of tar yields and the evolution of tar molecular weight distribution (MWD). Different tar weight classes are represented by finite lattice fragments of different numbers of sites. These finite fragments are formed when bridges and links in the infinite coal lattice are cleaved during pyrolysis. In the CPD model, concentrations of finite fragments are determined by the percolation theory based on instant intact bridge/link fraction of the infinite lattice. The Coal Kinetics Module, on the other hand, treats any finite fragment with a given number of sites as a unique surface species residing on the metaplast surface phase. Accordingly, the breaking off of finite fragments from the infinite lattice is considered as a surface reaction. A bulk species will be automatically recognized as a finite fragment when it is specified as a bulk species product of a fragmentation surface reaction. Molecular weights of bulk fragment species will be used as the bin-center values when the tar MWD is constructed. Because of the surface chemistry association, the Coal Kinetics Module requires the fragment production rates rather than the fragment concentrations to be calculated from the percolation statistics model. The fragment production rates can be derived from the percolation theory as a function of net bridge/link breakage rate and the number of sites in the finite fragment species. The concentration of a finite fragment will then be determined by the conservation law. A detailed description of the fragmentation surface reaction is presented in Section 3.3.4.

Concentrations of gas-phase tar species are computed by the distillation model. The flash distillation model of CPD and FLASHCHAIN assumes that vaporization and condensation of tar species occur instantaneously, such that the liquid-vapor equilibrium is maintained at all times [33, 35]. Once tar concentrations in the metaplast are known, the assumption of vapor-liquid equilibrium, i.e., Raoult's law, will establish the relationship between bulk fragment concentration and that of its corresponding tar species in the gas phase. Since tar fragments in the metaplast are bulk species, the Coal Kinetics Module treats the evaporation of a liquid tar species as a desorption reaction. By making liquid tar evaporation a reaction, we can relax the assumption of fast evaporation/condensation by the flash distillation model. Desorption rate parameters of surface/liquid tar species can be specified explicitly in the surface mechanism to introduce the appropriate distillation time scale. The mapping between liquid and gaseous tar species is also implied by the desorption reaction, as the corresponding gas-phase tar species would appear on the product side of the reaction. The vapor pressure of liquid/surface tar species, if it is known, can be specified as a property tag within the thermodynamic data processed by CHEMKIN. 
Otherwise, the vapor pressure correlation developed by Unger and Suuberg [41] will be applied by default. The Module's distillation model will compute the reverse (adsorption) rate such that Raoult's law is satisfied at equilibrium. The distillation model is described further in Section 3.3.4.

The CHEMKIN species approach for coal devolatilization modeling has a number of advantages. Coagulation of tar species can be conveniently implemented by borrowing the idea behind the Simultaneous Particle and molecule modeling (SPAMM) for aerosol simulations [42]. SPAMM is a variation of the sectional method for modeling aerosol size distribution (ASD). The novelty of SPAMM lies in its use of species to represent a section (or bin) of the ASD. Interactions between members from different sections, such as coagulation, can therefore be expressed as elementary reactions between the involved representative species. By assigning appropriate rate parameters, these reactions can be added to gas-phase and surface mechanisms to address coagulation of tar species in the gas phase and in the metaplast, respectively. Similarly, cracking of tar species might be included in simulations by introducing additional reactions. The other benefit of the species approach is that it adds flexibility to the coal thermal cracking (or bridge/bond cleaving) reaction scheme. The original CPD model employs a simple reaction sequence to represent the devolatilization process [33]. It begins with the slow activation of a labile bridge to a reactive bridge intermediate. The activated bridge will undertake two competing reaction paths. It can decompose into two side chains and thus disconnect the sites; or it can condense into a char link and release light gases during this process. The side chain will eventually break down to form gases due to thermal cracking. This devolatilization sequence is interpreted into a series of surface reactions in the Coal Kinetics Module. Alternative and/or new reaction pathways can thus be easily introduced to further advance our understanding of the coal devolatilization process.

Surface reaction types commonly utilized in the Coal Kinetics Module are presented in Figure 35. Graphical descriptions of these reactions are also provided as visual aids. The coal devolatilization reaction pathways together with the lattice structure model employed by the Coal Kinetics Module are illustrated in Figure 36. When concentrations of tar species in the metaplast and in the gas phase are known, it becomes straightforward to construct the tar's MWD in the Coal Kinetics Module. Figure 37 illustrates the assembly of a liquid tar MWD from concentrations of surface fragment species. Obviously, the number of surface fragment species and their molecular weights would affect the resolution of the tar MWD. 


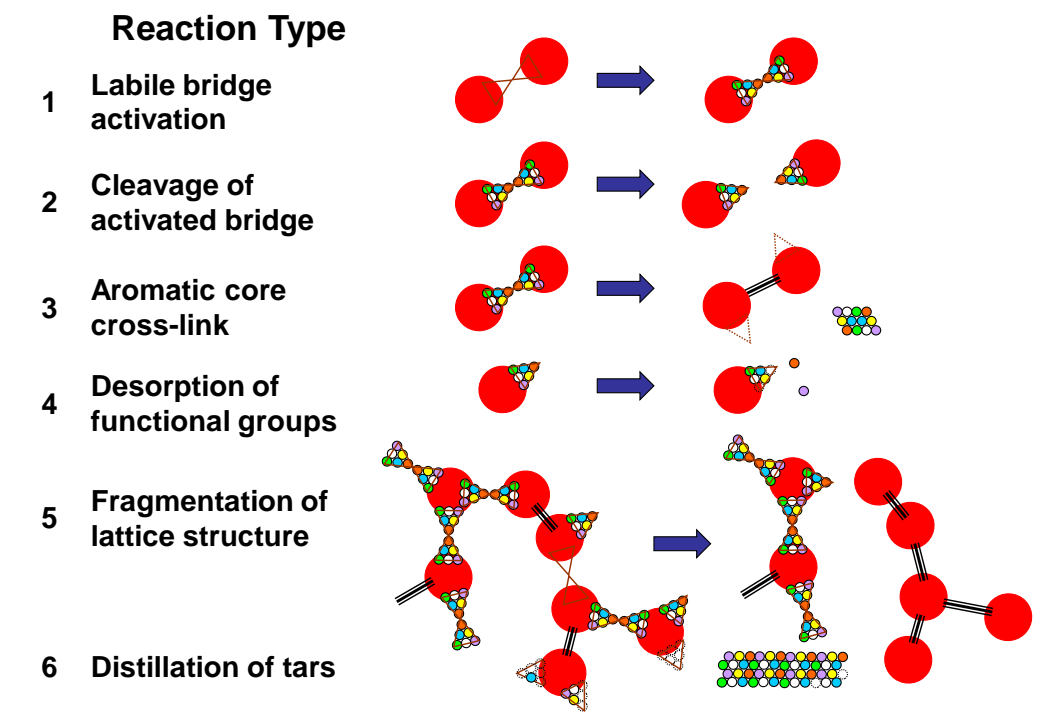

Figure 35. Devolatilization-specific surface reaction types in the Coal Kinetics Module.

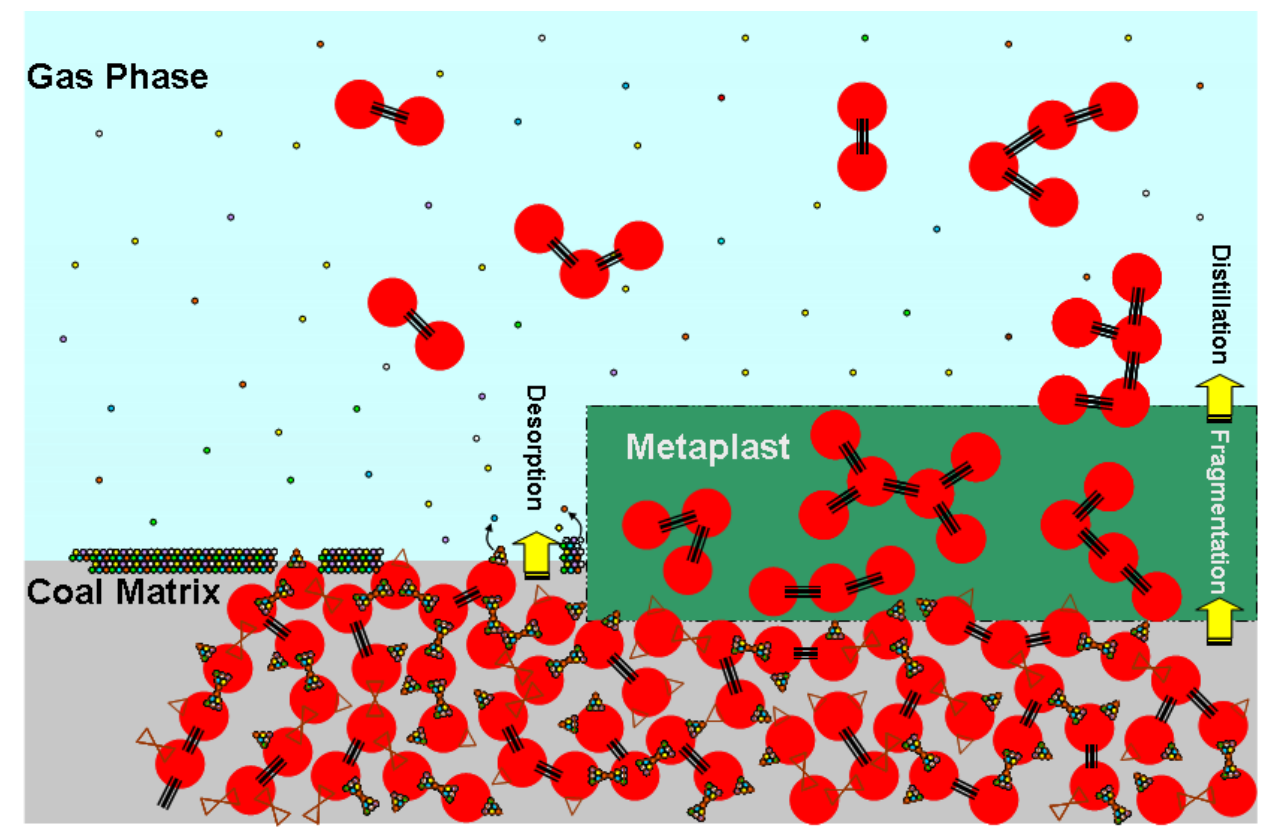

Figure 36. Graphical description of coal lattice model and major reaction pathways of coal devolatilization. 


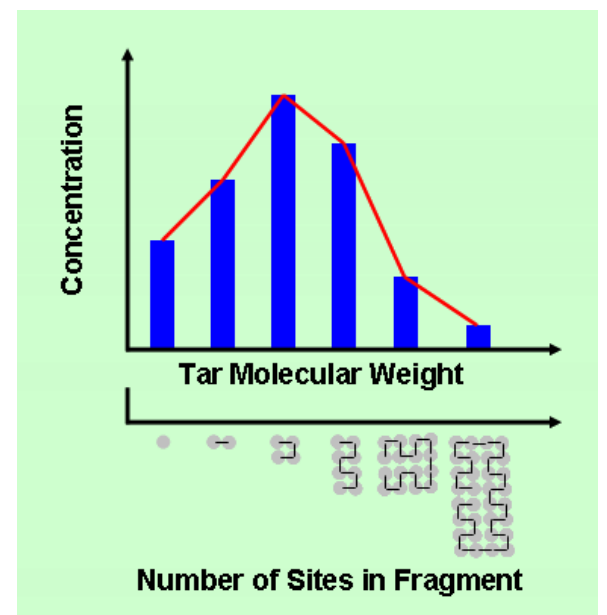

Figure 37. Construction of tar molecular weight distribution from fragment/tar species concentrations in the Coal Kinetics Module.

\subsubsection{Use of Coal Structural and Chemical Data}

Coals of different ranks and geographical sources exhibit diverse physical and chemical characteristics. Even coal samples from the same seam show substantial variations in behavior during devolatilization and combustion. Hence, a coal model must be able to describe the variety of coals. The Coal Kinetics Module characterizes coals in two aspects: structure and chemistry.

Coal-dependent structural parameters include the number of attachments per cluster, the number of bridges and loops, and the average molecular weights of aromatic clusters and side chains. The coal structure strongly influences its behavior during thermal cracking and the tar yields and weight distribution. The Coal Kinetics Module assumes that the underlying chemical kinetics for bridge scission, cross-linking, and desorption of functional groups and tars are invariant amongst different coal ranks and sources. Thus, from a chemistry perspective, the Coal Kinetics Module simply distinguishes coals by their elemental makeup and functional-group composition. Many useful coal-model parameters, especially those structural parameters, are determined from Nuclear Magnetic Resonance (NMR) analysis. However, the time and expense of NMR study limits the availability of such data, and only a small number of coal samples have been analyzed with this technique. In efforts to extend the applicability of coal modeling to any coal, various correlations have been developed. These correlations enable the estimation of a coal's structural parameters from its elemental composition [31, 32, 36, 43]. That is, the more easily obtained proximate and ultimate analysis data are necessary to run such models. Some of the correlations are derived by fitting structural parameters from a selected group of coal samples to polynomials of elemental mass fractions $[32,36]$. Others utilize a coal library and interpolate structural parameters as well as functional-group composition based on $\mathrm{H} / \mathrm{C}$ and $\mathrm{O} / \mathrm{C}$ ratios [31, 43].

We expect that correlations of a coal's structural parameters and its elemental composition, where available, are too complicated to be represented by a few factors and are likely to be highly nonlinear. Linear [36] or quadratic [32] functional relationships by forced empirical fits might therefore grossly undermine the true complexity of the system. A coal library [31], even with only limited resolution, offers a more suitable approach, since it is able to preserve the inherent complexity and can easily be expanded with new data. Without a good understanding of the underlying physics, another plausible method to estimate coal structural parameters from elemental composition would be the use of an artificial neural network (ANN). In theory, the ANN's ability to learn from examples offers the possibility of modeling complex systems of which controlling parameters or rules are not known in detail or are difficult to 
analyze. Given a well-behaved data set, ANN can be trained to produce reasonable outputs in response to new inputs. While this may be promising for future investigation, we chose not to pursue the ANN approach under the scope of this project, since it has not been previously tried and proven to be effective for this application.

The coal properties required by the Coal Kinetics Module are commonly available. These are:

1. Dry-ash-free (daf) data from coal ultimate analysis, i.e., elemental composition $(\mathrm{C}, \mathrm{H}, \mathrm{O}, \mathrm{N}$, S).

2. Coordinate number $(\sigma+1)$.

3. Average aromatic core molecular weight $M_{\text {core }}$.

4. Average side chain molecular weight $M_{g}$.

5. Intact bridge fraction $p_{0}$, which can be either computed by the Module itself, interpolated from a coal library or, if available from 13C NMR analysis, input directly. In this way, such inputs are provided by the user as optional inputs.

When the coal library is used, an area-weighted two-dimensional interpolation scheme is employed to look up parameters of the unknown coal from three nearest-neighboring library coals, according to their $\mathrm{H} / \mathrm{C}$ and $\mathrm{O} / \mathrm{C}$ ratios [31]. If a coal's 13C NMR data are available, the Module can calculate necessary coal structural parameters directly from the data. The calculation requires the mass fraction of carbon $\% \mathrm{C}$ from ultimate analysis and coordinate number, fraction of intact bridges, fraction of carbon atoms in aromatic bond $f_{a}^{\prime}$, and aromatic carbons per cluster $C A / C L$ from 13C NMR analysis [33].

\subsubsection{Model Equations and Relationships}

The average molecular weight of an aromatic core can be found by

$$
M_{\text {core }}=(C A / C L) M_{C}
$$

where $M_{C}$ is carbon atomic weight. Using the ratio of the number of aromatic carbons per cluster to the carbon aromaticity [44], the average molecular weight of an entire cluster can be obtained as

$$
M_{\text {cluster }}=\frac{(C A / C L) M_{C}}{f_{a}^{\prime} \cdot \% C}
$$

$\% \mathrm{C}$ is carbon atom mass fraction in the coal from ultimate analysis, which is always required by the Coal Kinetics Module. Assuming that all available connection terminals on the cluster are either occupied by a side-chain species or by a labile bridge and that a side-chain species is one half of a bridge species, the average molecular weight of side chain becomes:

$$
M_{\delta}=\frac{M_{\text {cluster }}-(C A / C L) M_{C}}{(\sigma+1)}=\left(\frac{1-f_{a}^{\prime} \% C}{\sigma+1}\right) M_{\text {cluster }} .
$$

Let $m_{\text {pellet }, i}$ be the initial mass of a coal pellet. The pellet mass can also be computed from

$$
m_{\text {pellet }, i}=X_{\text {core }, i} M_{\text {core }}+X_{L B, i} M_{L B}+X_{S C, i} M_{\delta}
$$


where $X_{\text {core, } i}, X_{L B, i}$, and $X_{S C, i}$ are the initial number of moles of bulk species: aromatic core, labile bridge, and side chain, respectively, per coal pellet. Since the Module assumes one labile bridge molecule is equivalent to two side chain molecules, the labile bridge molecular weight can be obtained as

$$
M_{L B}=2 M_{\delta} .
$$

In addition, the Bethe pseudo lattice assumption provides relationships between the number of sites and the number of bridges as well as the number of terminals:

$$
X_{\text {bridge }} N_{a v o}=X_{\text {site }} N_{a v o}-1
$$

and

$$
X_{\text {termin al }} N_{a v o}=(\sigma+1) X_{\text {site }} N_{a v o}-2 X_{\text {bridge }} N_{a v o} .
$$

Since the initial intact bridge fraction $p_{0}$ is known from inputs or correlations, the initial number of labile bridges can be found as

$$
X_{L B, i} N_{a v o}=p_{0} X_{b r i d g e, i} N_{a v o}=p_{0}\left(X_{c o r e, i} N_{a v o}-1\right)
$$

and thus the initial number of side chains becomes

$$
X_{S C, i} N_{a v o}=(\sigma+1) X_{c o r e, i} N_{a v o}-2 X_{L B, i} N_{a v o} .
$$

Substituting Equations (7), (10), and (11) into the pellet mass equation, Equation (6), allows the initial number of cores to be determined as

$$
m_{\text {pellet }, i}=X_{\text {core }, i} M_{\text {core }}+(\sigma+1) X_{\text {core }, i} M_{\delta}
$$

or

$$
X_{\text {core }, i}=\frac{m_{\text {pellet }, i}}{\left[M_{\text {core }}+(\sigma+1) M_{\delta}\right]} .
$$

Accordingly,

$$
X_{L B, i}=p_{0}\left(X_{\text {core }, i}-1 / N_{a v o}\right)
$$

and

$$
X_{S C, i}=(\sigma+1) X_{\text {core }, i}-2 X_{L B, i}
$$

The initial number of char links $X_{C L, i}$, which is used by the Module to compute total number of intact bridges/links in the coal lattice, is assumed to be zero.

Because all bulk species densities are specified when they are defined in the surface reaction mechanism, the initial bulk density of coal pellet can be determined by 


$$
\frac{1}{\rho_{\text {bulk }, i}}=\frac{1}{m_{\text {pellet }, i}} \sum_{k b=1}^{N_{\text {bulk }}}\left(\frac{X_{k b, i} M_{k b}}{\rho_{k b}}\right)
$$

and the initial pellet volume becomes

$$
V_{\text {pellet }, i}=m_{\text {pellet }, i} / \rho_{\text {bulk }, i} \text {. }
$$

The initial "class" of the coal pellet, needed when the Particle Tracking module is also activated, is represented by the number of bulk core molecules in the pellet:

$$
\text { Class }_{i}=X_{\text {core }, i} N_{a v o}
$$

The Coal Kinetics Module does not use the side chain bulk species directly. Rather, it replaces the side chain with a set of functional group bulk species. The mass fractions of these functional group species are obtained from coal library and are fixed throughout the simulation.

The initial functional-group species' mole numbers are determined by their mass fractions $Y_{F G, k}^{o}$ and the "average" molecular weight of side chain, $M_{\delta}$.

$$
X_{F G k, i}=\left(n_{F G} Y_{F G, k}^{o} M_{\delta} / M_{F G, k}\right) X_{S C, i}
$$

where the functional group fitting factor is given by:

$$
n_{F G}=M_{\delta} \sum_{k=1}^{N_{F G}}\left(Y_{F G, k}^{o} / M_{F G, k}\right)
$$

Evolution of core, labile bridge, char link, and functional-groups bulk species concentrations are always tracked by solving the conservation equations:

$$
\frac{d X_{k}}{d t}=\sum_{j} \dot{b}_{k, j}
$$

$\dot{b}_{k, j}$ is the molar production rate of bulk species $k$ by the $j^{\text {th }}$ surface reaction and is determined by surfacechemistry routines in CHEMKIN according to its rate formulation.

The Coal Kinetics Module uses surface reactions to control the progress of devolatilization. The reaction pathways of coal devolatilization start with thermal cracking of the coal matrix. The thermal cracking scheme in CPD [33] is implemented in the "starter" devolatilization surface mechanism:

$$
\begin{aligned}
& \mathrm{L}(\mathrm{B}) \rightarrow \mathrm{L}^{*}(\mathrm{~B}) \\
& \mathrm{L}^{*}(\mathrm{~B}) \rightarrow 2\left[\sum n_{F G} Y_{F G, k}^{o} F G_{k}(\mathrm{~B})\right] \\
& \mathrm{L}^{*}(\mathrm{~B}) \rightarrow \mathrm{C}_{\mathrm{L}}(\mathrm{B})+2\left[\sum n_{F G} Y_{F G, k}^{o} F G_{k}(\mathrm{~B})\right] .
\end{aligned}
$$

The labile bridge $\mathrm{L}(\mathrm{B})$ connecting cores/sites becomes reactive when it is heated. The activated bridge $\mathrm{L}^{*}(\mathrm{~B})$ can undergo two possible reaction paths. The reactive bridge could decompose into two side-chain macro-molecule complexes $\left[\sum n_{F G} Y_{F G, k}^{o} F G_{k}(\mathrm{~B})\right]$ attached to their respective cores. Or, the active bridge 
would stabilize to form a permanent char bridge $C_{L}(B)$ and squeeze out the side-chain complexes in the process. The char link $\mathrm{C}_{\mathrm{L}}(\mathrm{B})$ is a pseudo bulk species representing the bonding between two aromatic rings and has no elemental composition. It is included implicitly when the Coal Kinetics Module is activated and does not need to be declared as a real bulk species in the surface mechanism.

The side chain complex, which is a solid mixture of functional-group bulk species, will break free individually from the core surface into the gas phase. This light-gas release process is given as a set of desorption reactions in the surface mechanism:

$$
F G_{k}(\mathrm{~B}) \rightarrow G_{k} .
$$

The tar/fragment formation in the metaplast/surface is governed by the percolation theory based on the intact bridge fraction of the coal lattice. The Module uses surface fragmentation reactions to represent the surface tar formation process

$$
n \text { Core(B) } \rightarrow \text { Tar_n(B) }
$$

The stoichiometric coefficient $n$ of the core species Core(B) indicates the number of sites in the fragment Tar_ $n(\mathrm{~B})$. The rate of fragment reaction is computed internally by the Module according to percolation theory and values of $n$, coordinate number $(\sigma+1)$, and the intact bridge fraction $p=\left(L(B)+C_{L}(B)\right) /\left(L(B)_{0}+C_{L}(B)_{o}\right)$ as:

$$
\dot{b}_{\text {Tar } \_^{n}}=\frac{[\mu-\eta p]}{p(1-p)} \frac{\left[d L(B) / d t+d C_{L}(B) / d t\right]}{\left(L(B)_{o}+C_{L}(B)_{o}\right)} Q_{n}(p) \text { Core }_{o},
$$

where

$$
Q_{n}(p)=\frac{\sigma+1}{n \eta} \frac{\Gamma(\eta+1)}{\Gamma(\mu+1) \Gamma(\eta-\mu+1)} p^{\mu}(1-p)^{n(\sigma-1)+2}
$$

is the number density of $n$-site fragment for every site in the lattice. Hence, $Q_{n}(p)$ Core $_{o}$ is the number of mole of $n$-site fragment species. $\Gamma$ is the standard gamma function, $\eta=n \sigma+1$, and $\mu=n-1$ in the above equation.

After tar species are formed in the metaplast, they can be released to the gas phase by evaporation. The Module treats the evaporation/distillation of bulk tar species as a special type of desorption reaction. The distillation reaction is a reversible reaction and is given as

$$
\text { Tar_n(B) } \rightarrow \text { Tar_n }
$$

The forward rate of distillation reaction should be provided explicitly in the reaction mechanism to introduce a characteristic time scale for the evaporation of the tar species. The Coal Kinetics Module will compute the reverse rate to satisfy the Raoult's law at equilibrium.

\subsubsection{Software Implementation Considerations}

A multi-stage implementation plan was devised for the Coal Kinetic Module. The first phase involved developing software components that would allow the empirical functional-group approach to work. These tasks included: 
1. Recognizing "core", "bridge", and "regular" bulk species in the CHEMKIN surfacechemistry input.

2. Implementing of Distributed Activation Energy (DAE) reaction type in CHEMKIN surfacechemistry reaction routines.

3. Adding the concept of "elemental" bulk species that can be used to collect atoms from any unaccounted-for surface species due to coagulation in the Particle Tracking module.

4. Solving all bulk species conservation equations in the Particle Tracking module.

5. Allowing particle bulk density to be determined by a group of bulk species in the Particle Tracking module.

6. Turning off particle coagulation and aggregation in the Particle Tracking module when the Coal Kinetics Module is in use.

The second stage of implementation was the creation of the new generalized CPD model components. Experimental data for the coal library was then collected and a template for the coal devolatilization surface mechanism was assembled.

Next, the coal kinetics module was implemented and tested in a transient batch-reactor model. This was later extended for testing in PSR and PFR simulations. Finally, user-interface components were designed and planned, to allow the use of the Coal Kinetics Module through the CHEMKIN-PRO guided interface. While full implementation of the Coal Kinetics Module user interface was not part of the scope of this project, a detailed implementation plan was formulated and sufficient implementation was in place to allow proof-of-concept testing.

\section{Graphical User Interface Components}

When coal-specific features are used in the surface mechanism, the CHEMKIN pre-processor module will add a flag to the surface-chemistry linking file. The CHEMKN-PRO user interface will display the "Coal Properties" tab in the "Reactor Properties" panel if it detects the presence of the "coal" flag (see Figure 38). The "Coal Properties" tab will always prompt the user for ultimate analysis data, i.e., the elemental composition of the coal. In addition, an option is provided for entering coal structural parameters manually. When this option is selected, the user must provide the following inputs:

1. Coordinate number $(\sigma+1)$

2. Fraction of intact bridges $p_{0}$

3. Fraction of carbon atoms in aromatic bond $f_{a}^{\prime}$

4. Aromatic carbons per cluster $C A / C L$

All of these parameters can be obtained from ${ }^{13} \mathrm{C}$ NMR analysis of the coal. If this option is not selected, the internal coal library will be used to estimate the coal structural parameters. A prototype of the user interface components and their relationship to the core CHEMKIN components are shown in Figure 38.

One important aspect of coal simulations is the prediction of tar molecular weight distributions (MWD) in both gas and bulk phases. The surface chemistry file will contain integer arrays of gaseous and bulk tar species indices and the species will be sorted by molecular weight in ascending order. The post-processor will then use these species index arrays to create tar MWDs, as shown in Figure 37. 


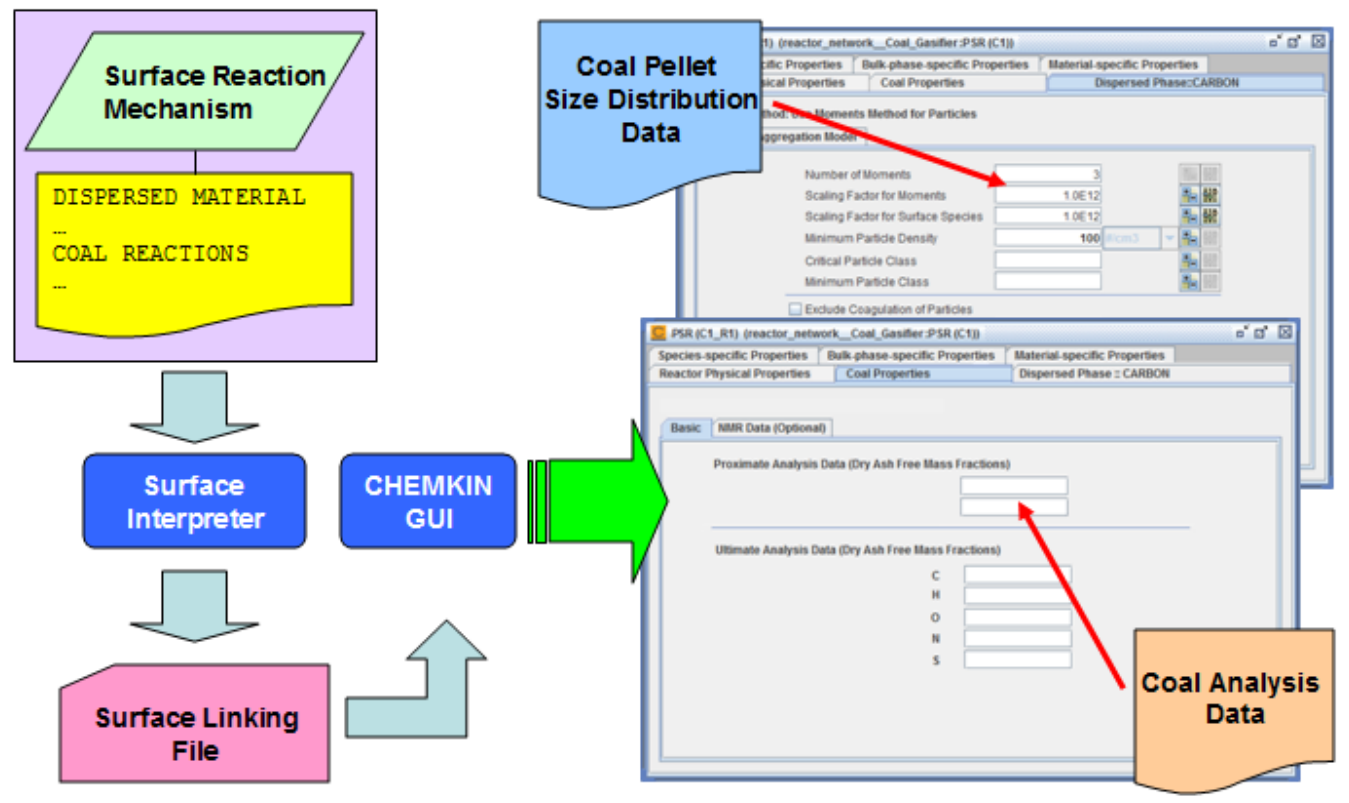

Figure 38. Activation of Coal and Particle Tracking properties panels in the CHEMKIN-PRO interface. 


\section{Results and Discussion}

As a way to demonstrate the results of the project, we focused on the demonstration of integrated results, using an NETL-provided single-stage gasifier CFD model. We tested the entire workflow, from the processing of the CFD model solution in ENERGICO, to the incorporation of detailed kinetics in the ERN, and then to the export and running of the model through the CAPE-OPEN UO interface in the Aspen Plus process flowsheet simulator. At each step of the way, we performed appropriate validation and verification of the results, to make sure that the software was functioning correctly. Finally, for the parallel work being performed to enhance the gasification kinetics capabilities, we performed unit and integration tests of the resulting Coal Kinetics Module as implemented in CHEMKIN-PRO.

\subsection{Equivalent Reactor Network for NETL Single-Stage Gasifier}

We started our integration and verification process with an updated 3-D CFD model of a single-stage coal gasifier, which was received from NETL during the project. When we first attempted to create an ERN for this gasifier, we discovered that some gas-phase species considered in the CFD model were not included in the original detail kinetics mechanism used by the ERN model. These gas-phase species represent volatiles of trace elements in the coal, such as sulfur. Although the CFD model does not include any gasphase reaction for these species, we thought it would be of interest to learn about their distributions as well as evolution throughout the gasifier system. Furthermore, we believe such a capability is one of the areas where the ERN model can be especially useful in extending the reduced-order model predictions beyond those of the original CFD model. To this end, we chose to expand the gas-phase reaction mechanism we were working with to include reaction pathways of minor coal volatiles, such as hydrogen sulfide $\left(\mathrm{H}_{2} \mathrm{~S}\right)$, carbonyl sulfide $(\mathrm{COS})$, hydrogen chloride $(\mathrm{HCl})$, and mercury $(\mathrm{Hg})$ species. In addition, we added the polycyclic aromatic hydrocarbon $(\mathrm{PAH})$ species and their reaction pathways. $\mathrm{PAH}$ species are important because they correspond to tar species released from the coal thermal-cracking process. In our Coal Kinetics Module (CKM), the tar (or PAH) species are formed through the vaporization of coal fragments in the metaplast. The inclusion of PAH species in the gas-phase mechanism allows the ERN model to track the coal tar species and to predict the possible formation of soot particles during the coal gasification process. These enhancements and the application of the enhanced model to the NETL singlestage gasifier model are described in more detail below.

\subsubsection{Gas-Phase Mechanism}

The original gas-phase mechanism used by the ERN model contained reactions involving C0 - C2 hydrocarbon and $\mathrm{NO}_{x}$ species. While this mechanism is adequate for describing interactions among major coal syngas components, larger hydrocarbons, especially PAHs, must be included in the mechanism to properly address the fates of tar/PAH species released during coal devolatilization. Accordingly, the improvement of the gas-phase mechanism for coal gasification processes involves two parts: reactions of $\mathrm{C} 0$ - $\mathrm{C} 4$ hydrocarbon compounds, $\mathrm{PAH}$, and $\mathrm{NO}_{\mathrm{x}}$ that can be extracted from a master mechanism and independent submechanisms of species containing coal trace elements that have to be compiled from the literature.

The expanded C0 - C4 hydrocarbon mechanism to be used in the coal syngas ERN model is extracted from a well-validated gasoline combustion mechanism containing both NOx and PAH reaction pathways. We perform the submechanism extraction by using Reaction Design's Reaction Workbench software. Since PAH species consist of at least 6 carbon atoms, a simple mechanism reduction based on 
the number of $\mathrm{C}$ atoms in a species will not work well in this case. Alternatively, mechanism reduction is done by forcing some key species to be kept in the final submechanism. These key species include selected alkane and alkene species up to $\mathrm{C} 4, \mathrm{HCN}, \mathrm{NO}$, and $\mathrm{NO}_{2}$ for $\mathrm{NOx}$ reactions, and benzene and pyrene for tar/PAH reactions. Also, the reductions are performed for conditions relevant to typical coal gasifier operations. The final submechanism for the hydrocarbon part contains 371 species and 2779 reactions.

The $\mathrm{SO}_{\mathrm{x}}$ submechanism (version 5.2) [45] developed at Leeds University serves as the basis of our sulfurrelated reactions. The Leeds $\mathrm{SO}_{x}$ mechanism is based on studies done at Leeds University [46, 47] and similar research by Alzueta et al. [48]. In addition, we added some important $\mathrm{H}_{2} \mathrm{~S}$ pyrolysis reactions suggested by Binoist et al. [49]. This $\mathrm{SO}_{x}$ submechanism includes all S-containing species considered by the NETL CFD gasifier model.

The mercury submechanism consists of two major components: mercury oxidation to mercuric chloride $\left(\mathrm{HgCl}_{2}\right)$ for high $\mathrm{Cl}$-content coals and mercury oxidation to mercuric oxide $(\mathrm{HgO})$ for low $\mathrm{Cl}$-content coals. The $\mathrm{HgCl}_{2}$ submechanism is based on the reactions recommended by Niksa [50], and is derived from the works by Widmer et al. [51] and Roesler et al. [52]. The $\mathrm{HgO}$ reactions are those reported in the studies of Edwards et al. [53] and Xu et al. [54].

We also considered reactions related to silicon dioxide $\left(\mathrm{SiO}_{2}\right)$. However, we were unable to identify any significant reactions involving $\mathrm{SiO}_{2}$ under the coal gasifier conditions.

With all of the submechanisms for coal trace elements compiled, they were then merged together with the $\mathrm{C} 4$ mechanism containing $\mathrm{NO}_{\mathrm{x}}$ and $\mathrm{PAH}$ reactions. The final C4-coal-syngas mechanism consists of 405 species and 2940 reactions. The mechanism can handle reactions between light hydrocarbon species up to $\mathrm{C} 4$; $\mathrm{PAH}$ growth up to pyrene, which is commonly considered as the soot precursor; $\mathrm{NO}_{\mathrm{x}}$ and $\mathrm{SO}_{\mathrm{x}}$ formation; and mercury speciation. We used this C4-coal-syngas mechanism in our ERN simulation of the latest NETL gasifier case.

\subsubsection{Equivalent Reactor Network Creation}

The general instruction for exporting the CGNS file from an ANSYS FLUENT simulation solution is described in the ENERGICO User Manual [2]. The steps for creating an ERN for a coal gasifier/combustor in ENERGICO are presented in detail in Section 3.1.3 of this report. In this final demonstration, we followed the same process to create an ERN for the NETL gasifier case. Here, we omit the detailed description of the ERN creation procedures and focus instead on the results.

\section{Zone Partition}

Using a filter algorithm in ENERGICO similar to that described in Section 3.1.3, we were able to divide the NETL gasifier model into a 34-zone reactor network. The zone partitioning is presented in Figure 39. A 34-zone partition was deemed to be adequate, because there is no flame structure to be resolved in the coal gasifier. 


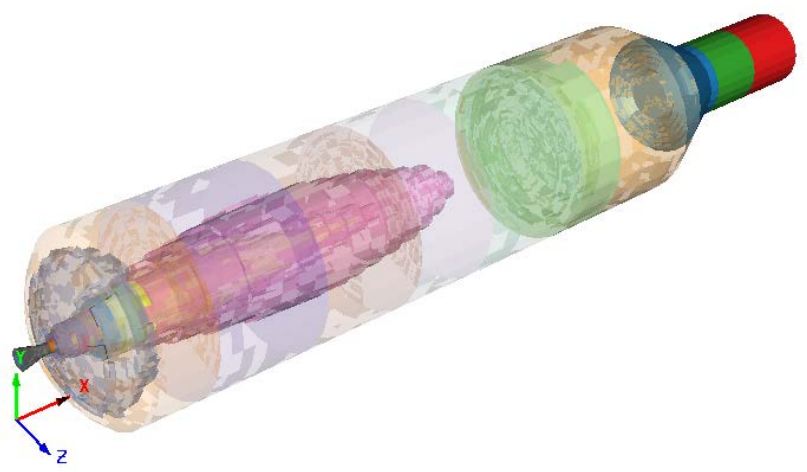

Figure 39. Zone partitioning of the latest NETL coal gasifier case.

\section{Equivalent Reactor Network}

The corresponding 34-zone ERN model of the coal gasifier is shown in Figure 40, as it is displayed within CHEMKIN-PRO, which is launched from ENERGICO after completion of the zone partitioning. The original ERN generated by ENERGICO did not have any tearing streams; it was instead a single "cluster" of PSRs that could be solved simultaneously. However, we intentionally de-clustered the original ERN into several smaller networks with tearing streams, within the CHEMKIN-PRO user interface, in order to speed up the calculation. This also allows us to test the tear-stream algorithm implemented in the CAPEOPEN encapsulation. The exact method of de-clustering the ERN should not affect the final solution, it is only expected to affect the compute time for the calculation, and thus is not an essential step.

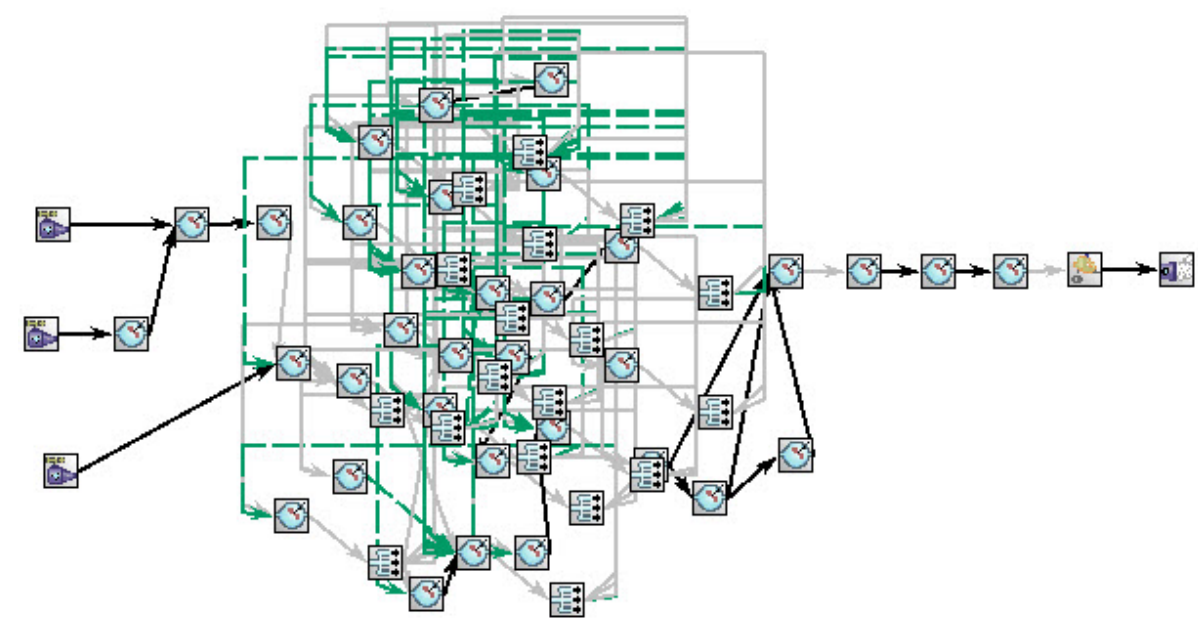

Figure 40. 34-zone equivalent reactor network model for the latest NETL coal gasifier case.

\subsection{Comparisons between the ROM and the Original CFD Model}

Once the ERN simulation is completed within CHEMKIN-PRO, the results can be mapped back to the 3-D gasifier geometry as viewed in the ENERGICO user interface. Here, we can visualize where different species are formed in the coal gasifier and also compare solutions of major species between the ERN and CFD simulations. This comparison tests the validity of the reduced-order model in representing the original CFD solution. 


\section{Major Species}

Both ERN and CFD solutions are in good agreement for major syngas species, such as $\mathrm{CH}_{4}$ (Figure 41 and Figure 42), $\mathrm{CO}$ (Figure 43 and Figure 44), and $\mathrm{H}_{2}$ (Figure 45 and Figure 46). In each of these sets of figures, the color legend is the same for both results. The larger difference in $\mathrm{H}_{2}$ results between ERN and CFD are an outcome of a portion of the $\mathrm{H}_{2}$ being consumed by $\mathrm{NO}_{x}$ and $\mathrm{SO}_{x}$ reactions that are included in the ERN simulation but that were not considered in the original CFD simulation.

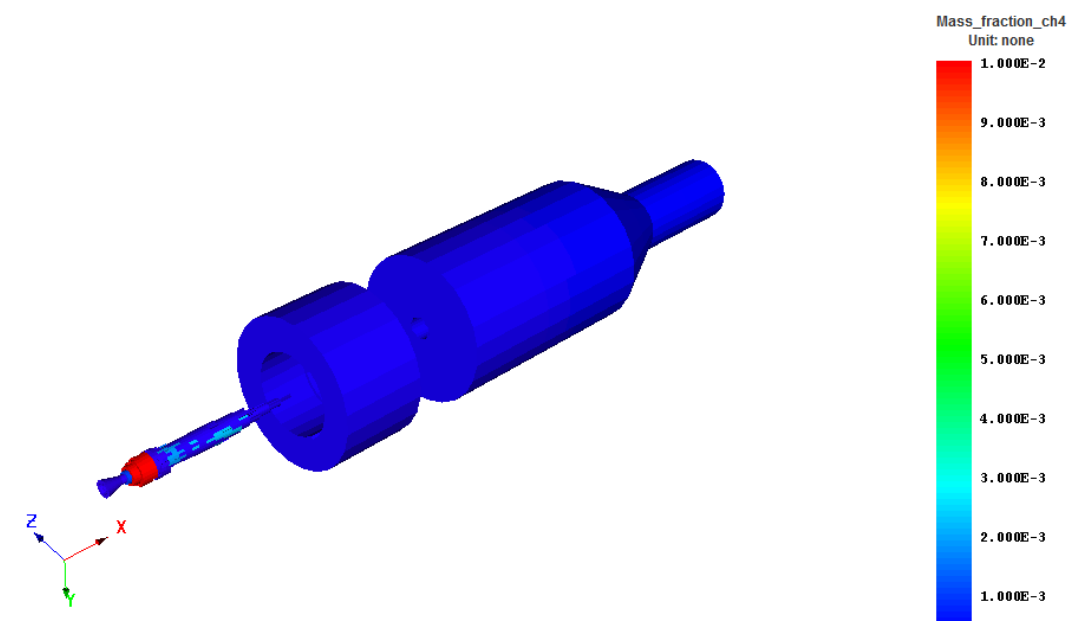

Figure 41. 34-zone ERN prediction of $\mathrm{CH}_{4}$ distribution inside the coal gasifier.

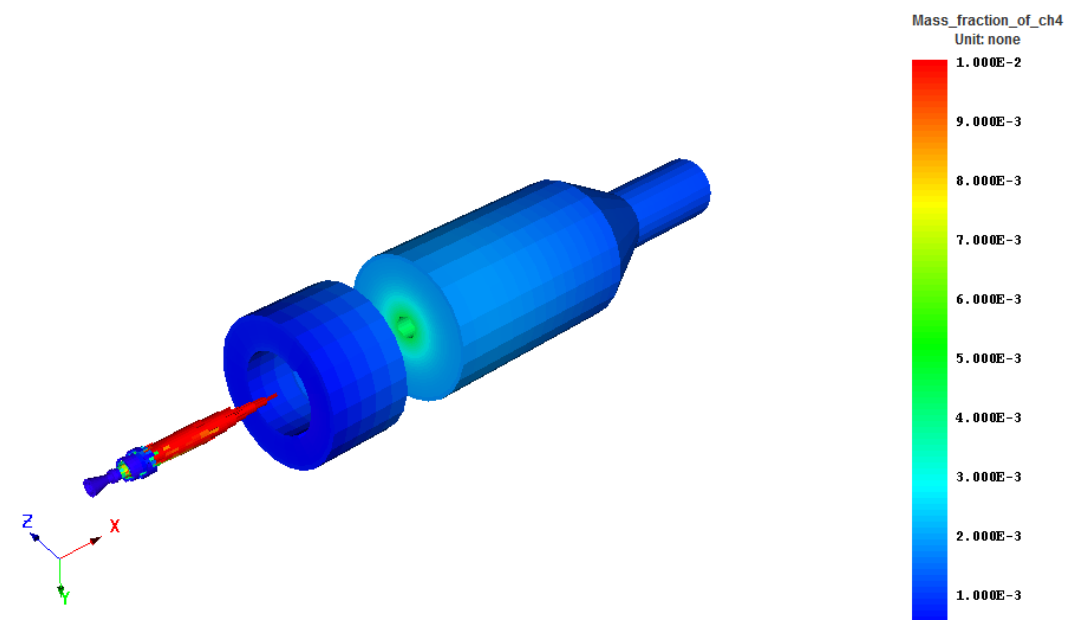

Figure 42. CFD prediction of $\mathrm{CH}_{4}$ distribution inside the coal gasifier. 


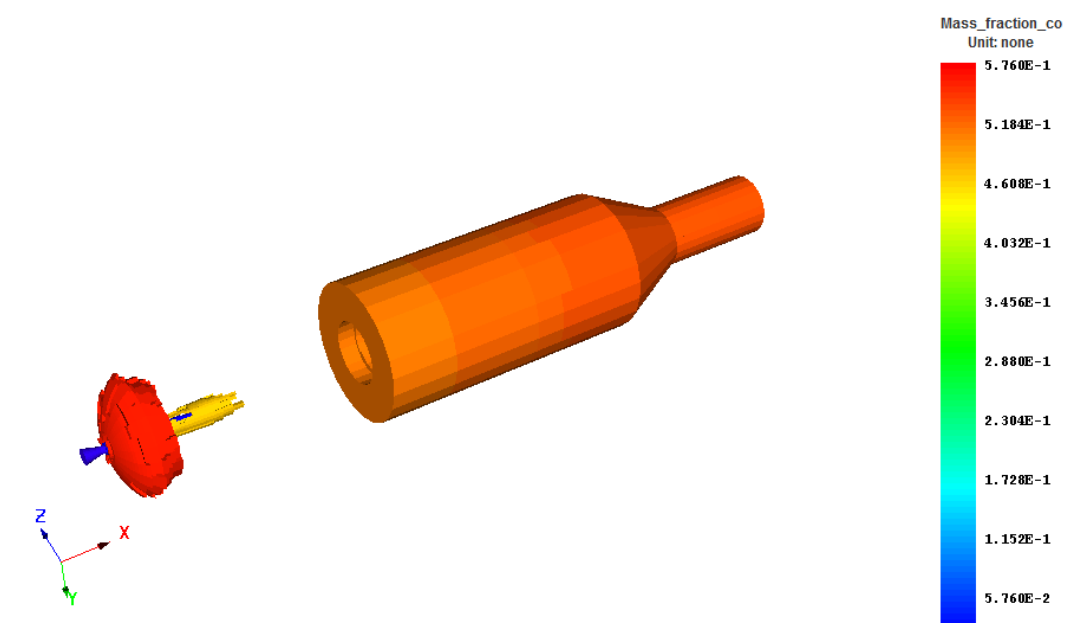

Figure 43. 34-zone ERN prediction of $\mathrm{CO}$ distribution inside the coal gasifier.

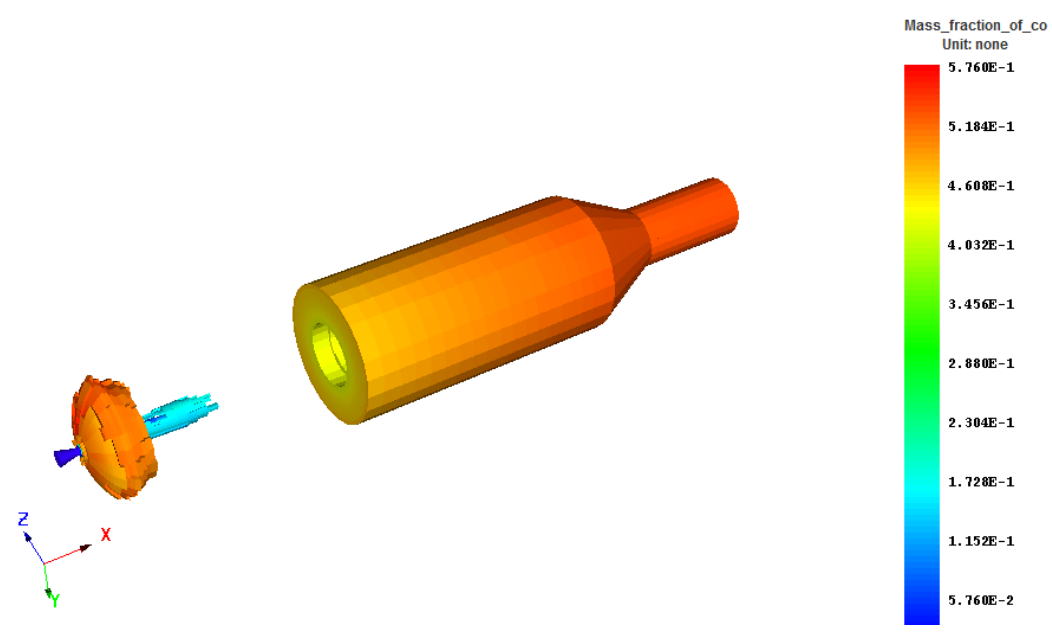

Figure 44. CFD prediction of CO distribution inside the coal gasifier. 


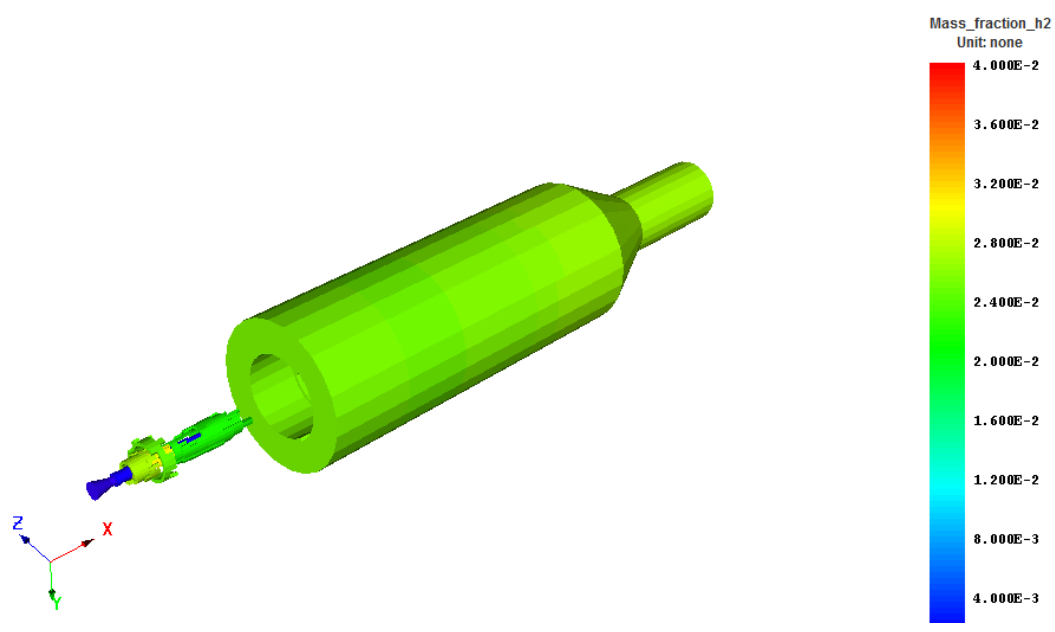

Figure 45. 34-zone ERN prediction of $\mathrm{H}_{2}$ distribution inside the coal gasifier.
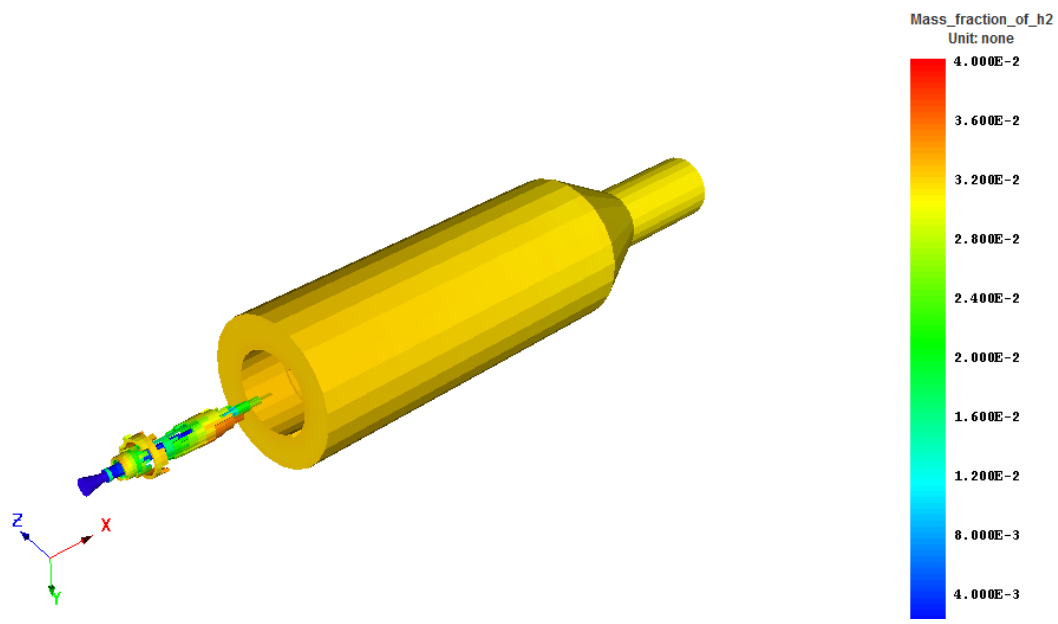

Figure 46. CFD prediction of $\mathrm{H}_{2}$ distribution inside the coal gasifier.

\section{TarlAromatic Species}

The benzene distributions predicted by both CFD and ERN models show differences in the region where significant devolatilization activity occurs. The differences can be attributed to the inclusion of PAHrelated reactions in the ERN simulation. Since the CFD simulation does not include any reaction involving benzene $\left(\mathrm{C}_{6} \mathrm{H}_{6}\right)$, the CFD result merely considers the transport of $\mathrm{C}_{6} \mathrm{H}_{6}$ in the coal gasifier. On the other hand, the ERN simulation considers reactions involving molecular-weight growth to form other PAH species. Benzene released from the coal can be quickly converted to other species via various gasphase reactions, depending on the conditions. In this case, CHEMKIN-PRO's Reaction Path Analyzer allows visualization of the dominant reaction pathways. As shown in Figure 49, most benzene is decomposed into smaller hydrocarbons. The lack of growth of benzene into larger PAHs is due to the low level of acetylene $\left(\mathrm{C}_{2} \mathrm{H}_{2}\right)$, the main ingredient of the HACA growth mechanism for PAH formation [55], in the system. 



Figure 47. 34-zone ERN prediction of benzene distribution inside the coal gasifier.

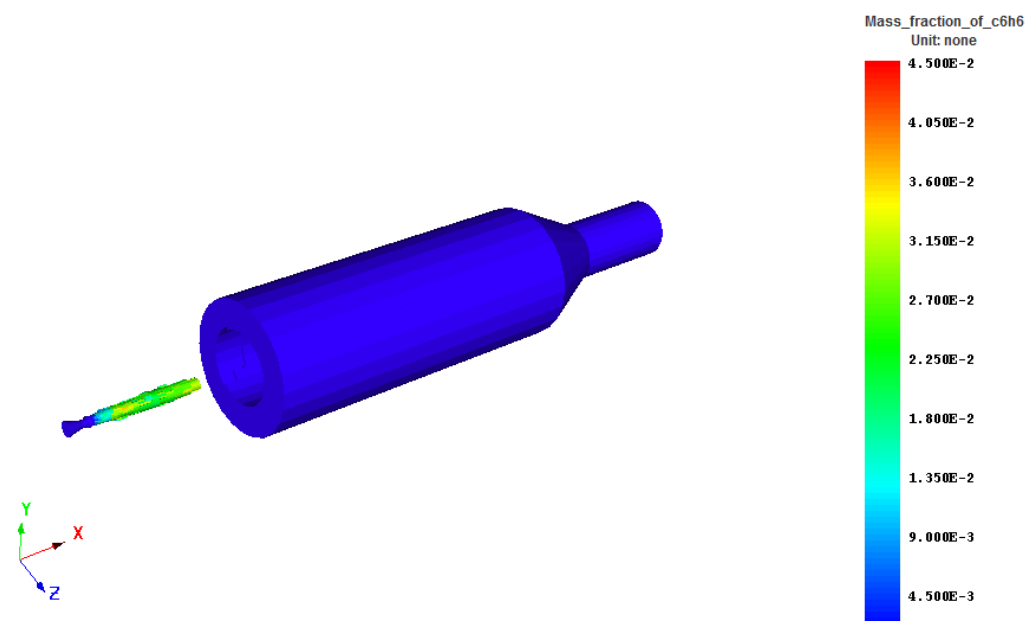

Figure 48. CFD prediction of benzene distribution inside the coal gasifier. 


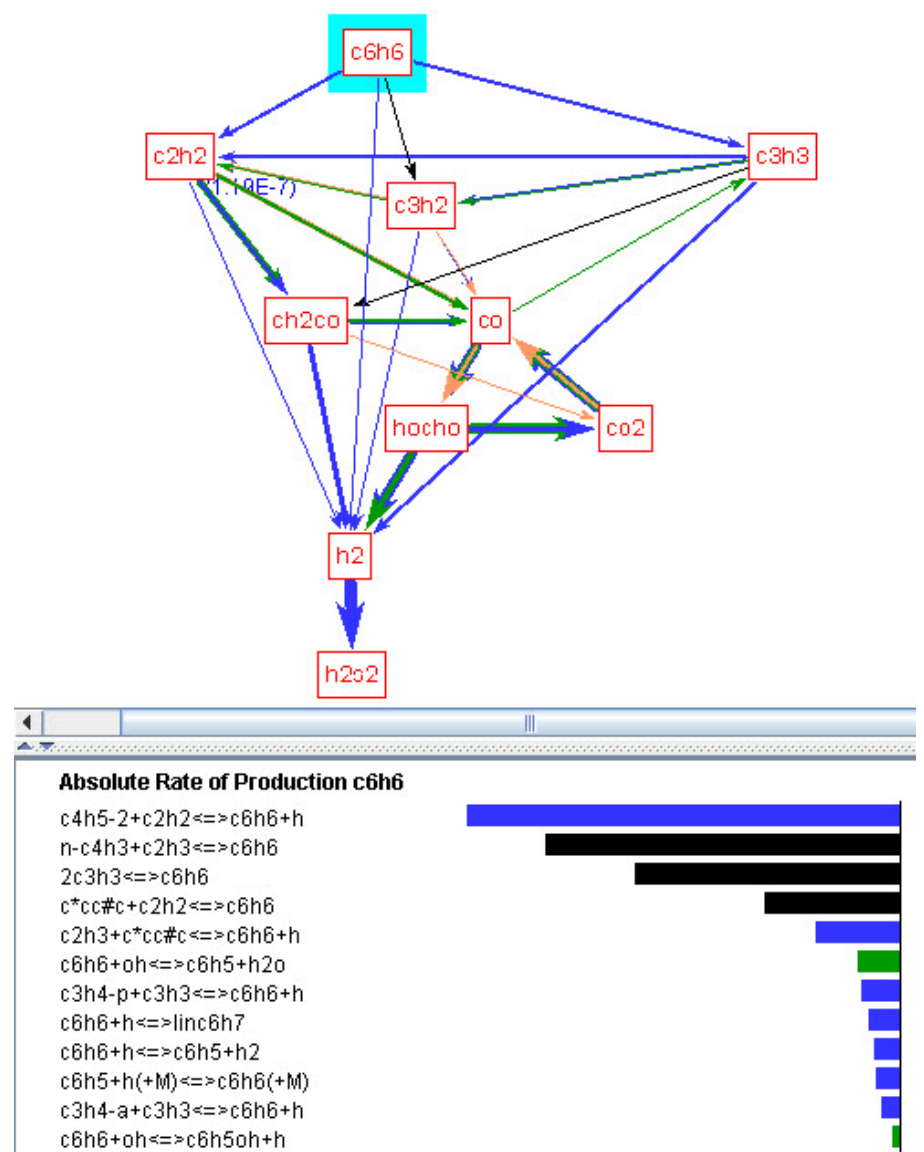

Figure 49. Reaction pathway diagram showing benzene being mainly decomposed into smaller hydrocarbons.

\section{NOx Formation}

The CFD simulation does not consider $\mathrm{NO}_{x}$ formation, such that only the ERN simulation can provide information about $\mathrm{NO}_{\mathrm{x}}$-related species in the gasifier. Distributions of $\mathrm{NH}_{3}$ and $\mathrm{NO}$ inside the gasifier, as predicted by the ERN model, are given in Figure 50 and Figure 51, respectively. Since the CFD devolatilization model assumes fuel nitrogen released from the coal is entirely in the form of $\mathrm{N}_{2}$, the $\mathrm{NO}_{x}$ formation due to the prompt- $\mathrm{NO}_{\mathrm{x}}$ route is not fully realized in the ERN simulation. Consequently, $\mathrm{NO}_{\mathrm{x}}$ emissions from the gasifier could be under-estimated. 


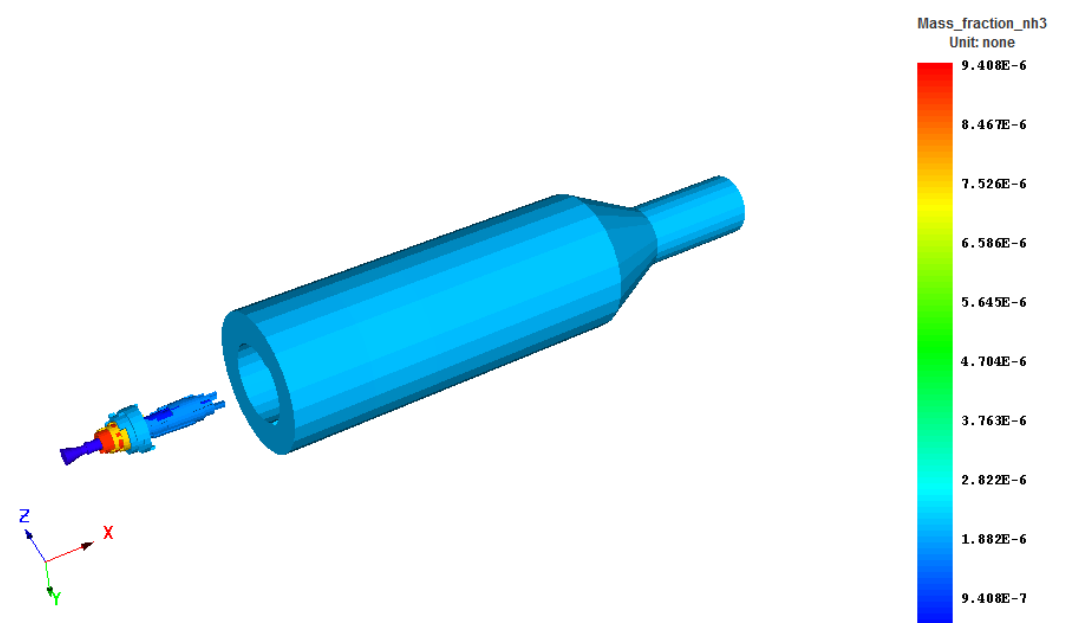

Figure 50. 34-zone ERN prediction of $\mathrm{NH}_{3}$ distribution inside the coal gasifier.
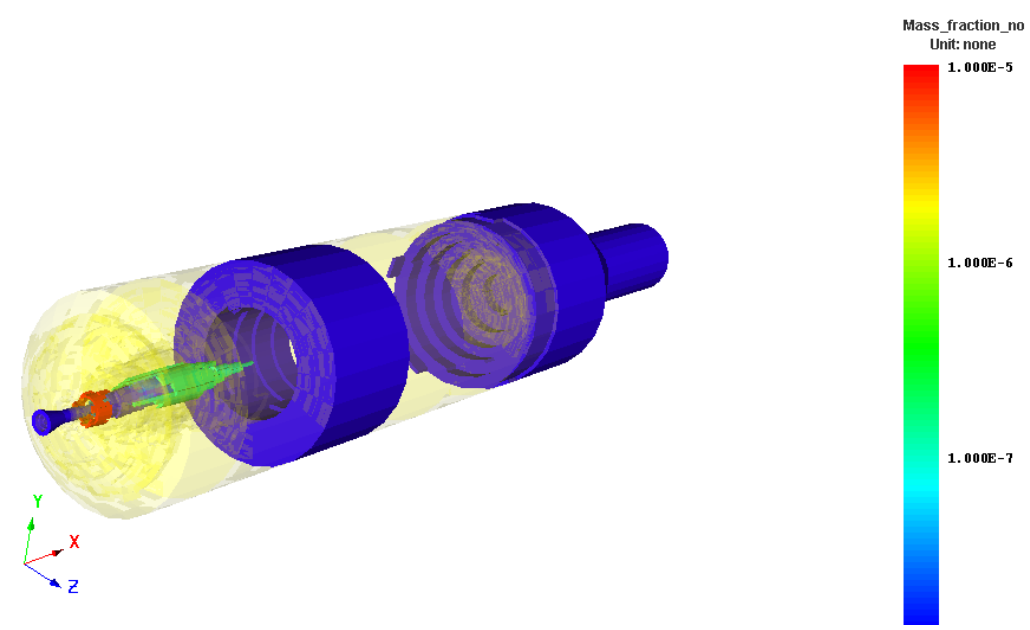

Figure 51. 34-zone ERN prediction of NO distribution inside the coal gasifier.

\section{Sulfur Species and SOx}

The CFD devolatilization model assumes sulfur content in the coal is released to the gas phase in the form of $\mathrm{H}_{2} \mathrm{~S}$. The predicted $\mathrm{H}_{2} \mathrm{~S}$ distribution from the ERN and CFD simulations are presented in Figure 52 and Figure 53, respectively. Without any $\mathrm{SO}_{x}$-related gas-phase reactions, the CFD simulation cannot predict $\mathrm{SO}_{x}$ emissions. The ERN simulation, however, is able to provide information on $\mathrm{SO}_{x}$ species inside the gasifier. Figure 54 gives the $\mathrm{SO}_{2}$ level predicted by the ERN model. In addition, the Reaction Path Analyzer in Figure 55 shows the paths by which $\mathrm{SO}$ and $\mathrm{SO}_{2}$ are formed from $\mathrm{H}_{2} \mathrm{~S}$. 


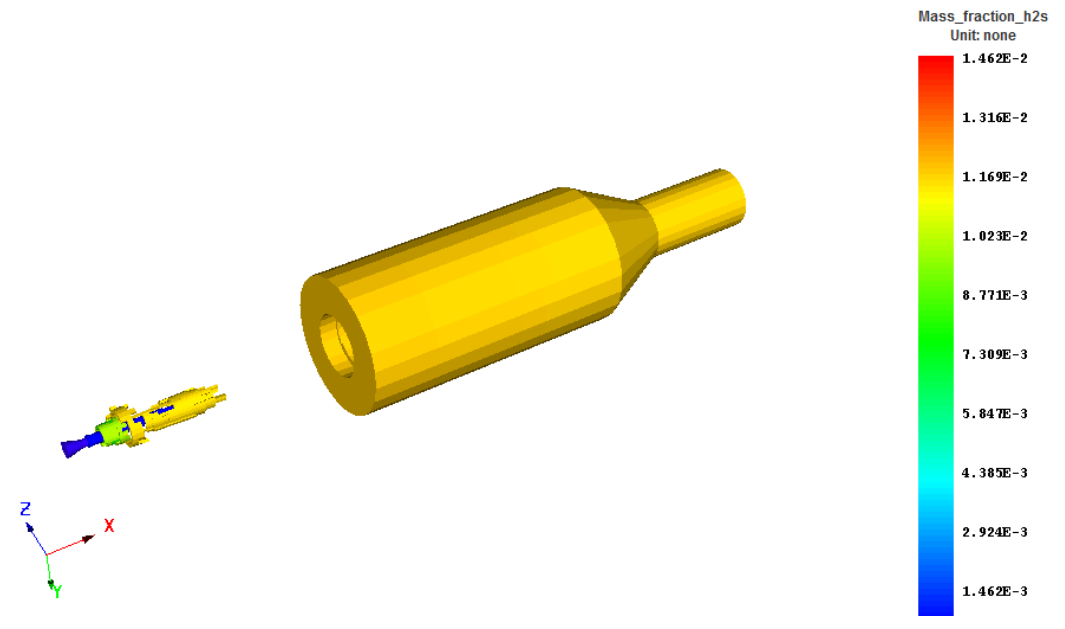

Figure 52. 34-zone ERN prediction of $\mathrm{H}_{2} \mathrm{~S}$ distribution inside the coal gasifier.



Figure 53. CFD prediction of $\mathrm{H}_{2} \mathrm{~S}$ distribution inside the coal gasifier. 


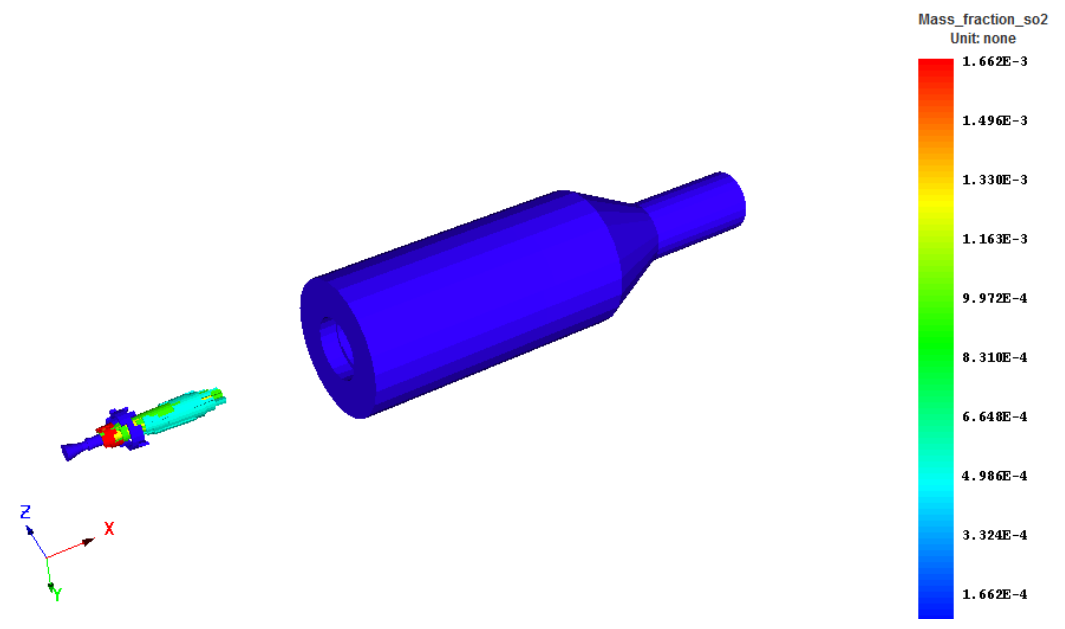

Figure 54. 34-zone ERN prediction of $\mathrm{SO}_{2}$ distribution inside the coal gasifier.
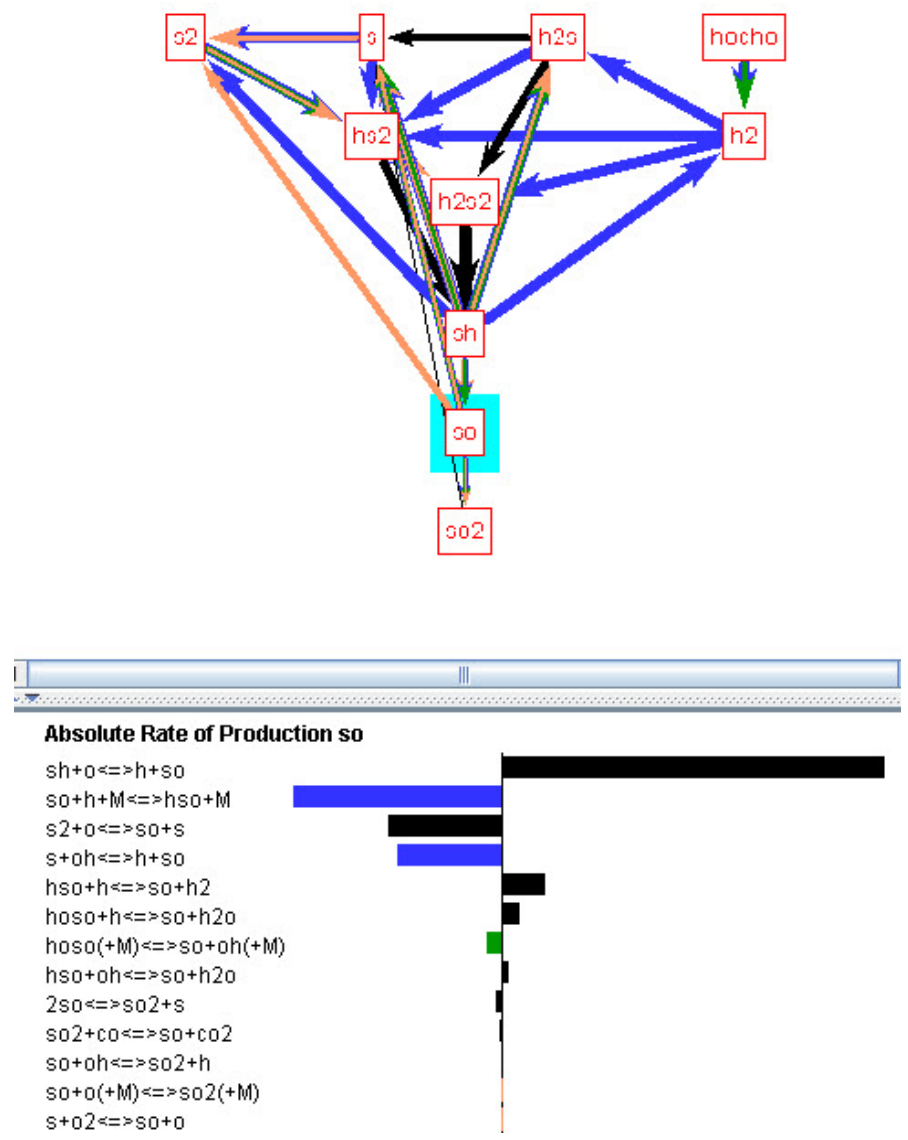

Figure 55. Reaction pathway diagram showing how $\mathrm{SO}_{2}$ is formed from $\mathrm{H}_{2} \mathrm{~S}$.

\section{Hydrogen Chloride}

Hydrogen chloride $(\mathrm{HCl})$ is another major coal volatile considered by the CFD devolatilization model. The $\mathrm{HCl}$ distribution predicted by the ERN model agrees well with that obtained by the CFD model, as shown in Figure 56 and Figure 57. 

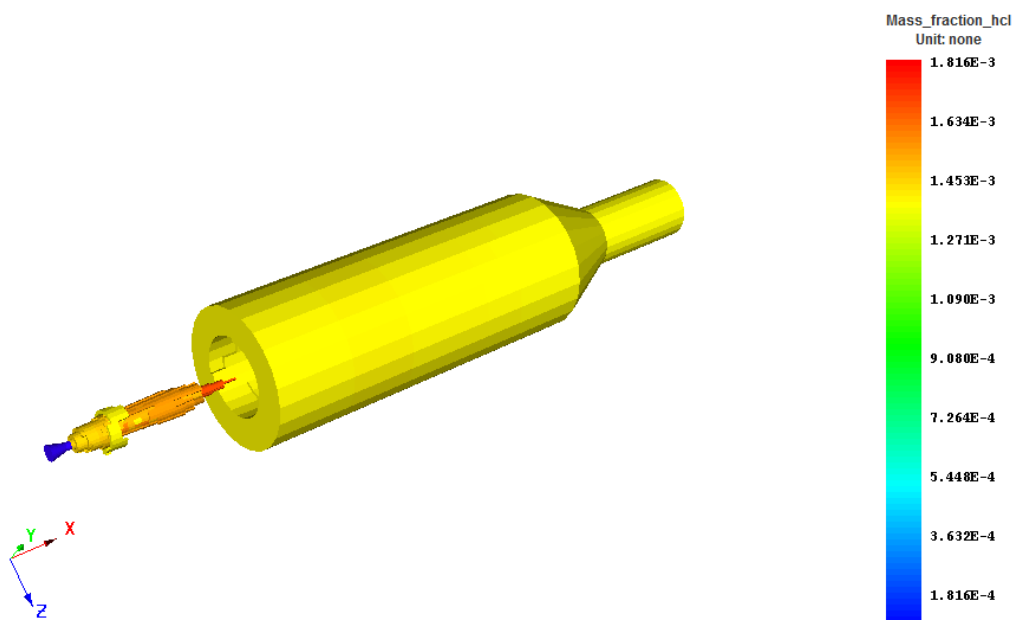

Figure 56. 34-zone ERN prediction of $\mathrm{HCl}$ distribution inside the coal gasifier.

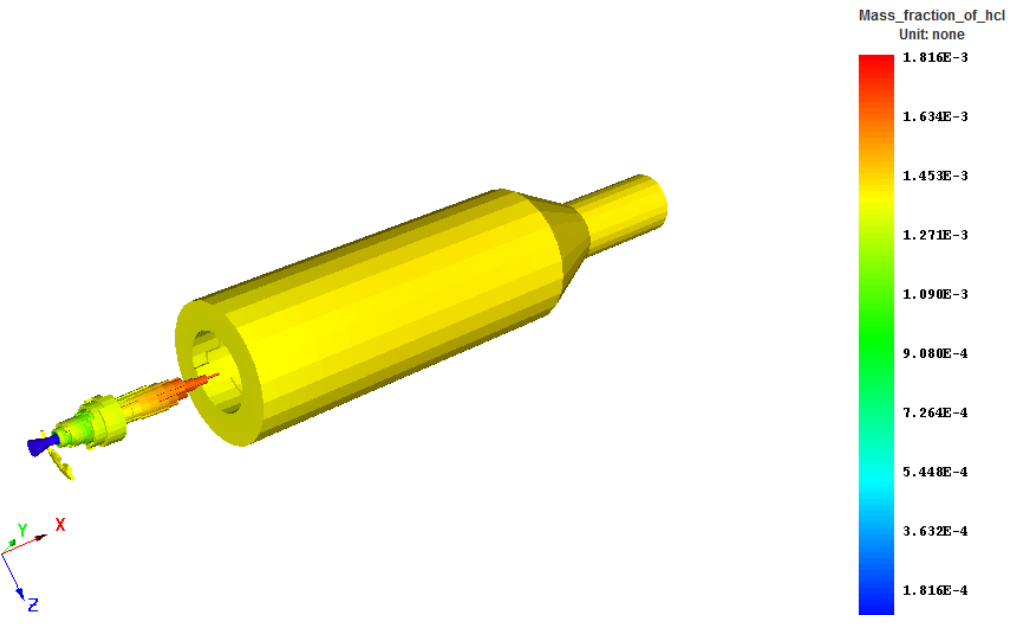

Figure 57. CFD prediction of $\mathrm{HCl}$ distribution inside the coal gasifier.

\subsection{Verification of the CAPE-OPEN Implementation}

The final validation test for the CAPE-OPEN implementation compared results of the single-stage gasifier ERN as run in the standalone CHEMKIN-PRO with the same ERN run within the COFE CAPE-OPEN compliant flowsheet simulator [27]. Inlet streams were hooked up to the CO Unit-Operation ERN to provide the same inlet information as were in the original inlets to the CFD model. The outlet of the ERN was connected back to the flowsheet to allow monitoring of the outlet state for the entire ERN.

The ERN model contained 371 species and 2779 reactions, while only 14 major species were tracked in the process simulator. In these simulations, both element balance and mass balance were strictly enforced in both the ERN and the COFE simulation, using the species-lumping method for trace species, when communicating back to the flowsheet. Figure 58 provides a comparison of the resulting output for this validation study. The same case was also verified using Aspen Plus [1]. The results show no noticeable impact on the accuracy of the results due to the lumping implementation. The good agreement also verifies the CO-interface implementation for the complex ERN that includes several tear streams, as shown in Figure 40. 


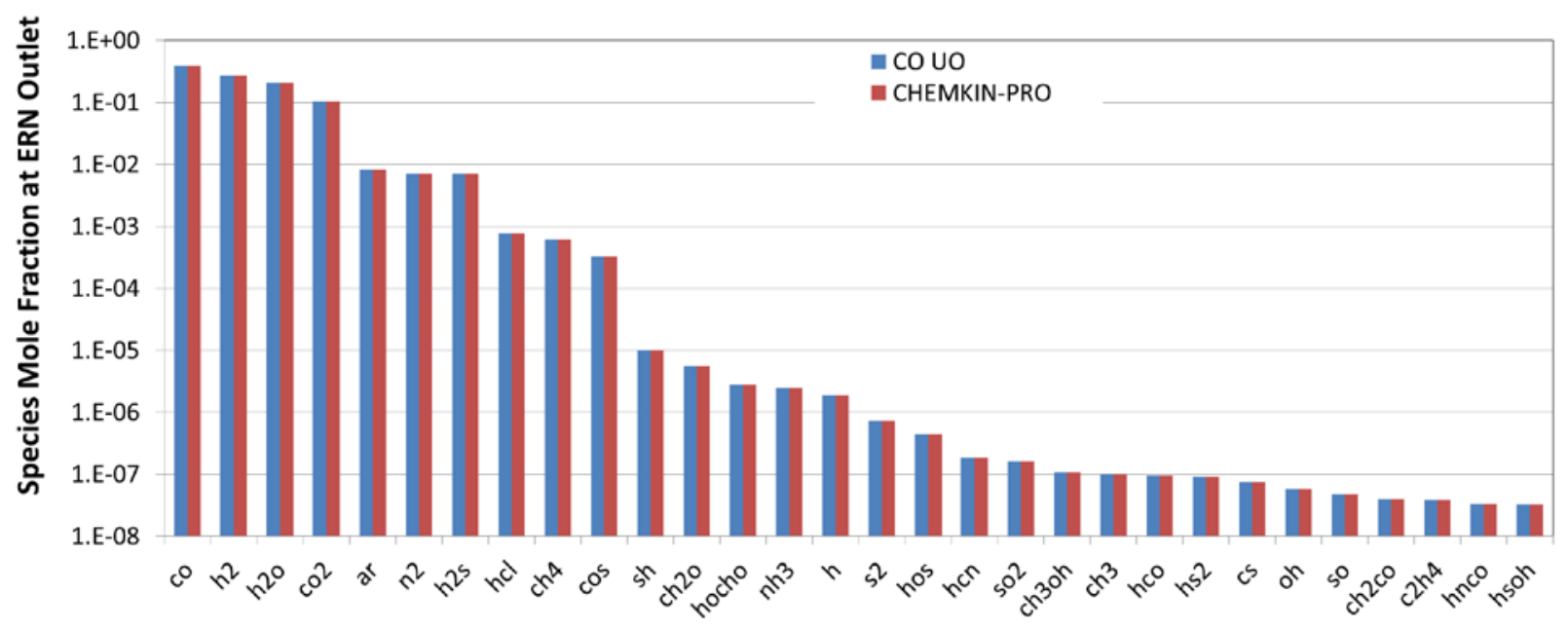

Figure 58. Comparison of CHEMKIN-PRO (standalone) results vs. results from the encapsulated $\mathrm{CO}$ Unit Operation for an ERN representing a single-stage gasifier.

\subsubsection{Performance Gains through CO Encapsulation}

We expected and verified that there was significant performance gain from running the ERN without input/output that is normally required with the standalone CHEMKIN-PRO software. Communicating instead via in-memory array passing through the dynamically linked library (DLL) that encapsulates the specific ERN configuration removes the computational cost of the I/O, which can be particularly important for an iterative process simulation. For one example simulation, CPU time for an ERN within CHEMKIN-PRO was 783 seconds, while simulation time on the same hardware for the CAPE-OPEN version of the ERN solution was 186 seconds. These compute times were for a 4-core Intel Xeon desktop PC. This nearly $4 x$ speedup further enhances the utility of the reduced-order model for plant simulations and opens the door for even more detailed kinetics description of the chemical processes represented.

\subsection{Chemical Percolation Devolatilization Model Testing}

In parallel to the ENERGICO enhancements, the development of the gasifier representation using ERNs, and the CAPE-OPEN encapsulation of the ERN for use in flowsheet simulations, we developed the initial Coal Kinetics Module framework and began our software-integration testing. We first implemented the CKM in the CHEMKIN-PRO batch-reactor model. With this implementation completed, unit tests were performed to verify that the integrated software functions correctly. These unit tests allowed us to ensure that each new coal-related reaction feature was working as designed and was producing physically reasonable values. We also used these tests to gain knowledge about the behavior and the new reactions that will inform future work on the Coal Kinetics Module.

Results from validation tests of several important coal-related reactions are presented below. All tests involve simulations using a transient constant-pressure and constant-temperature batch (closed homogeneous) reactor. This fundamental reactor type is the most important for evaluating chemical kinetics and is the work-horse of most kinetics model development. 


\subsubsection{Distributed Activation Energy Reaction}

For coal, the molecular structure and elemental composition of a functional group's macro-species, such as aromatic core, bridge, and peripheral structure, can vary substantially. Consequently, the rates of reactions involving these macro-molecules cannot simply be expressed by a static set of kinetic parameters. However, because the dependencies of the actual distributions of the kinetic parameters are unknown, the variation in kinetic rates may be attributed only to the variation of the activation energy, for simplicity.

The distributed activation energy (DAE) reaction [56] was therefore introduced to address the breakup of heterogeneous molecular structures (i.e., liable bridges) connecting aromatic cores in the coal. Typically, a Gaussian distribution with mean activation energy $\bar{E}$ and standard deviation $\sigma_{E}$ is assumed for the activation energy of the DAE reaction.

Figure 59 depicts the apparent activation energy, $E_{a}$, which increases as a function of reaction progress, and the effect of the standard deviation of activation energy $\left(\sigma_{E a}\right)$ on the evolution of the apparent $E_{a}$. At the start of the coal thermal-cracking process, molecular structures with relatively weak bonds will be broken first and thus we give the thermal-cracking reaction a relatively low activation energy $\left(E_{a}\right)$. As the thermal cracking progresses, bridges with low $E_{a}$ will be depleted and the apparent $E_{a}$ of the thermal cracking reaction begins to increase. A larger $\sigma_{E}$ results in a wider variation in the effective activation energy for a DAE reaction.

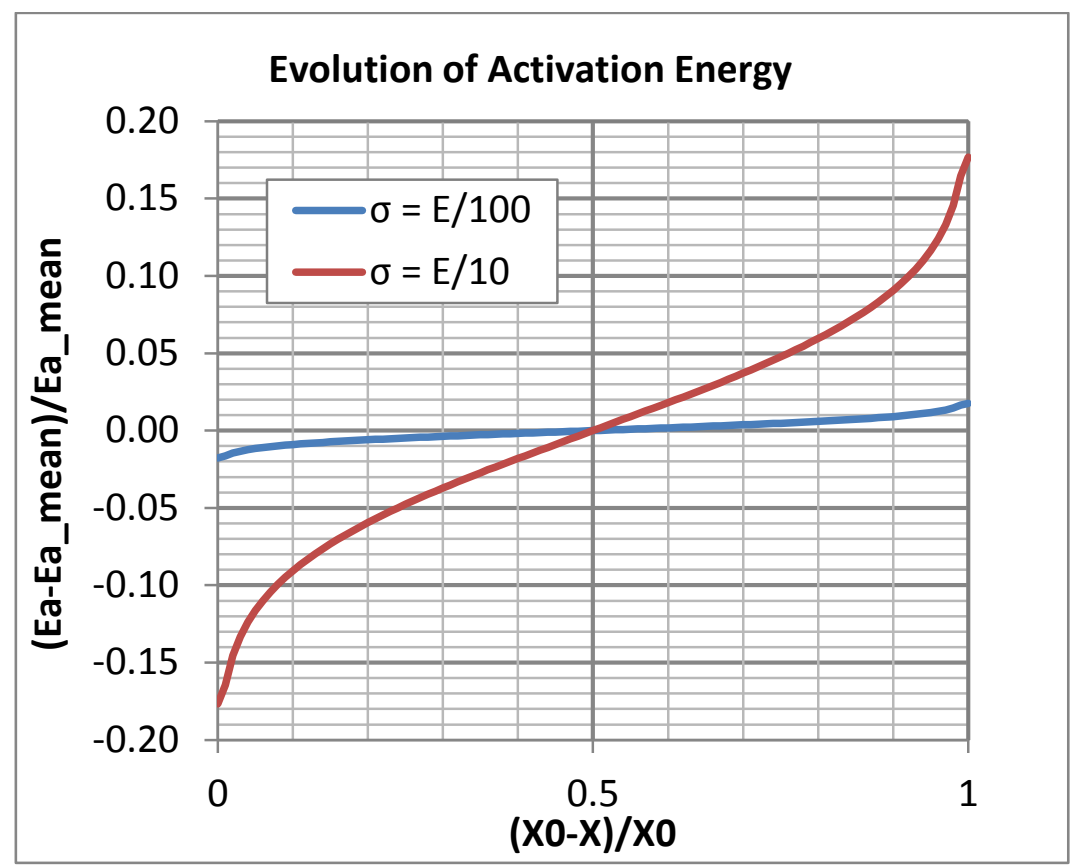

Figure 59. Evolution of actual activation energy as a function of reaction progress. The effect of standard deviation of activation energy is also showed.

Figure 60 and Figure 61 compare the effects of $\sigma_{E}$ on reactant mole fraction profiles for mean activation energies $\left(E_{a \text {,mean }}\right)$ of $1 \mathrm{kcal} / \mathrm{mole}$ and $50 \mathrm{kcal} /$ mole, respectively. For a regular reaction, the reactant decreases exponentially as the reaction progresses. The reactant profiles of DAE reactions, on the other hand, indicate the accelerated consumption of a reactant at the start of the reaction due to lower effective activation energies. The reactant consumption rate of the DAE reaction continues to decrease as the 
effective activation energy increases. The deviation from the regular reaction becomes more pronounced when $\sigma_{E}$ or $E_{a \text {.mean }}$ of the DAE reaction is large. When a DAE reaction with large $E_{a, \text { mean }}$ and large $\sigma_{E}$, the reactant profile, as shown by the $50 \mathrm{kcal} / \mathrm{mole}$ case in Figure 61, exhibits a long "tail" towards the completion of the reaction where the effective activation energy is much higher than the mean value.

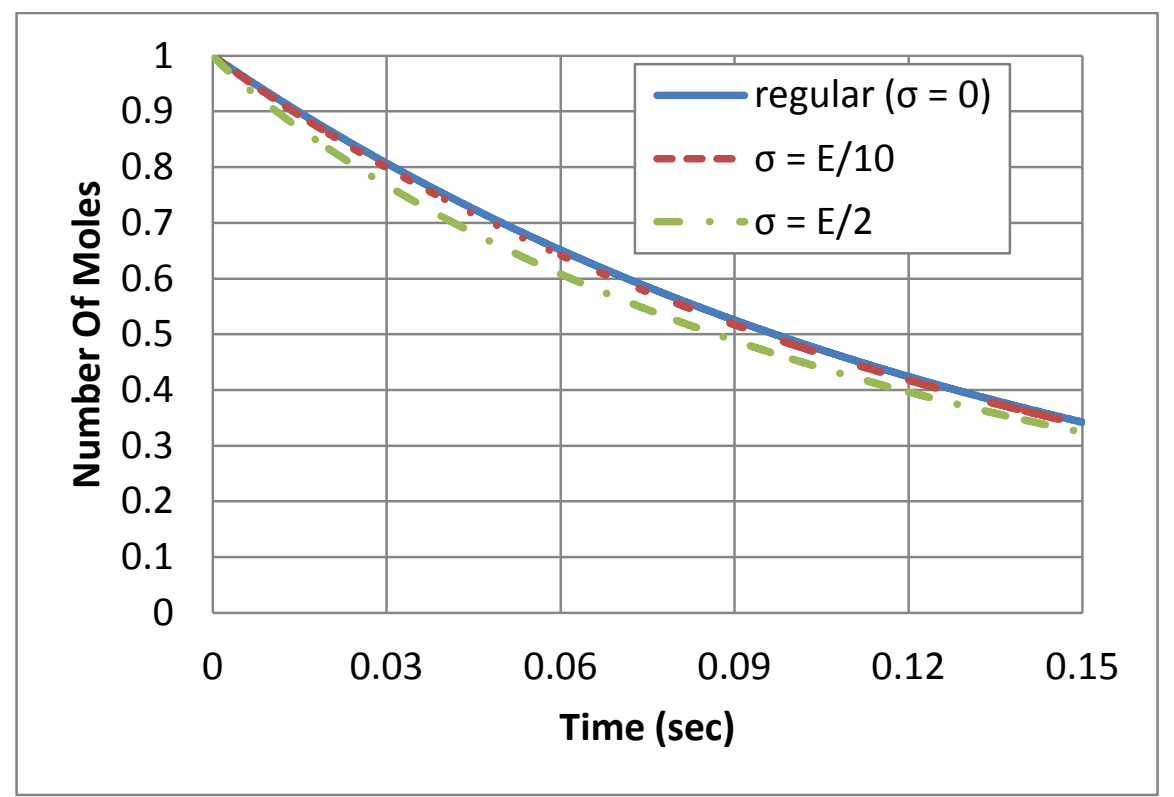

Figure 60. Comparisons of predicted reactant profile between non-DAE reaction and DAE reactions with a mean $E_{a}$ of $1 \mathrm{kcal} / \mathrm{mole}$ but different standard deviations.

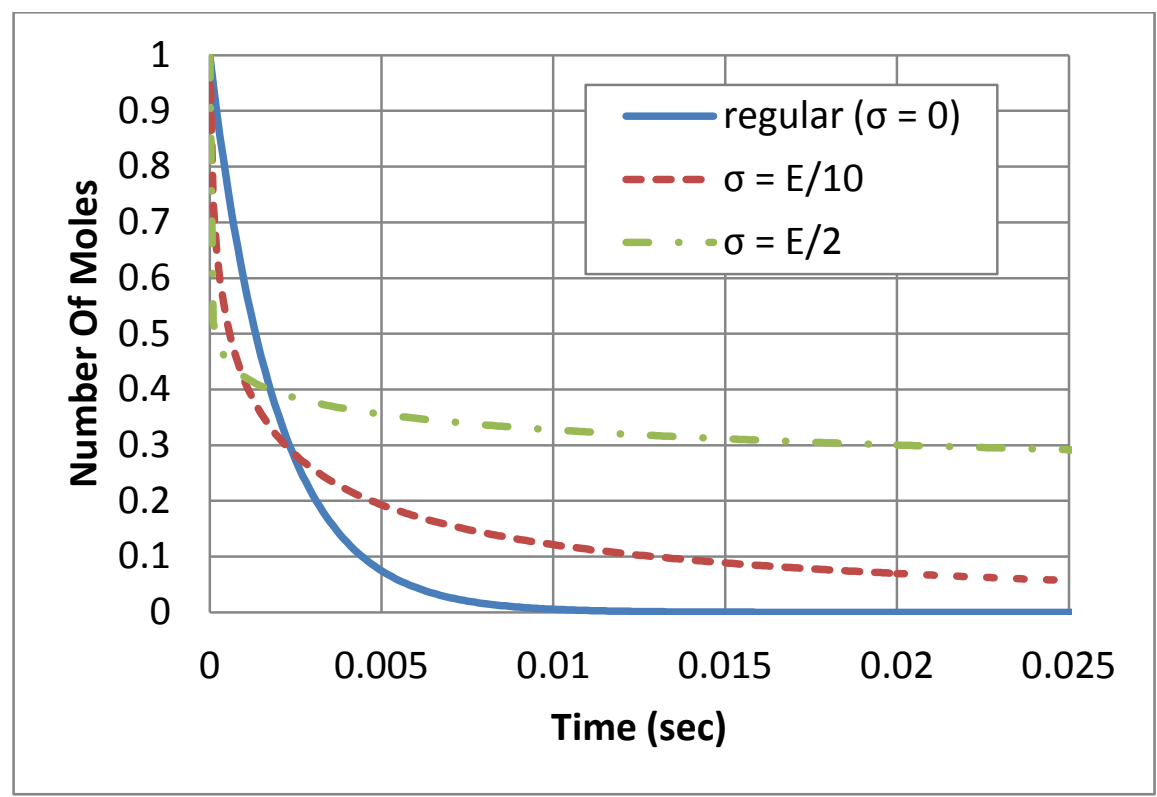

Figure 61. Comparisons of predicted reactant profile between non-DAE reaction and DAE reactions with a mean $E_{a}$ of $50 \mathrm{kcal} / \mathrm{mole}$ but different standard deviations. 


\subsubsection{Distillation Reaction}

The distillation reaction is a new type of surface reaction that can be applied to describe phase-change processes, such as tar release from coal devolatilization and fuel-droplet vaporization. The purpose of creating a distillation/vaporization "reaction" is to allow the otherwise static phase-equilibrium constraint to be incorporated into the dynamics of a reaction mechanism. The distillation/vaporization reaction will automatically enforce the vapor-liquid phase equilibrium described by Raoult's law.

We designed a validation test to make sure that the flash distillation reaction satisfies Raoult's law for multi-component systems. The liquid/metaplast in this test consists of five tar species with molar weights between 100 and $200 \mathrm{~g} /$ mole. At steady state, partial pressures of the vapor tar species predicted by the model should satisfy Raoult's law:

$$
x_{k} P=l_{k b} P_{k}^{v}
$$

$P_{k}^{v}$ is the vapor pressure of a gaseous tar species $k$ at a given temperature, while $x_{k}$ is the gas-phase mole fraction of species $k$, and $l_{k b}$ is the mole fraction (or bulk activity) of the corresponding bulk species in the bulk/liquid phase on which it resides, which is given by:

$$
l_{k b}=\frac{X_{k b}}{\sum_{i=1}^{N_{\text {frag }}} X_{i b}} .
$$

Here, $N_{\text {frag }}$ is the total number of bulk species in the same liquid/metaplast bulk phase as the vaporizing bulk species, i.e., all fragment bulk species, and $X_{i b}$ is the number of moles of bulk species $i$ in the liquid/metaplast bulk phase.

The steady-state mole fractions of both liquid tar species and their corresponding vapor species are presented in Figure 62. Figure 63 shows that the distillation reaction model yields solutions that automatically satisfy Raoult's law for multi-component systems. 


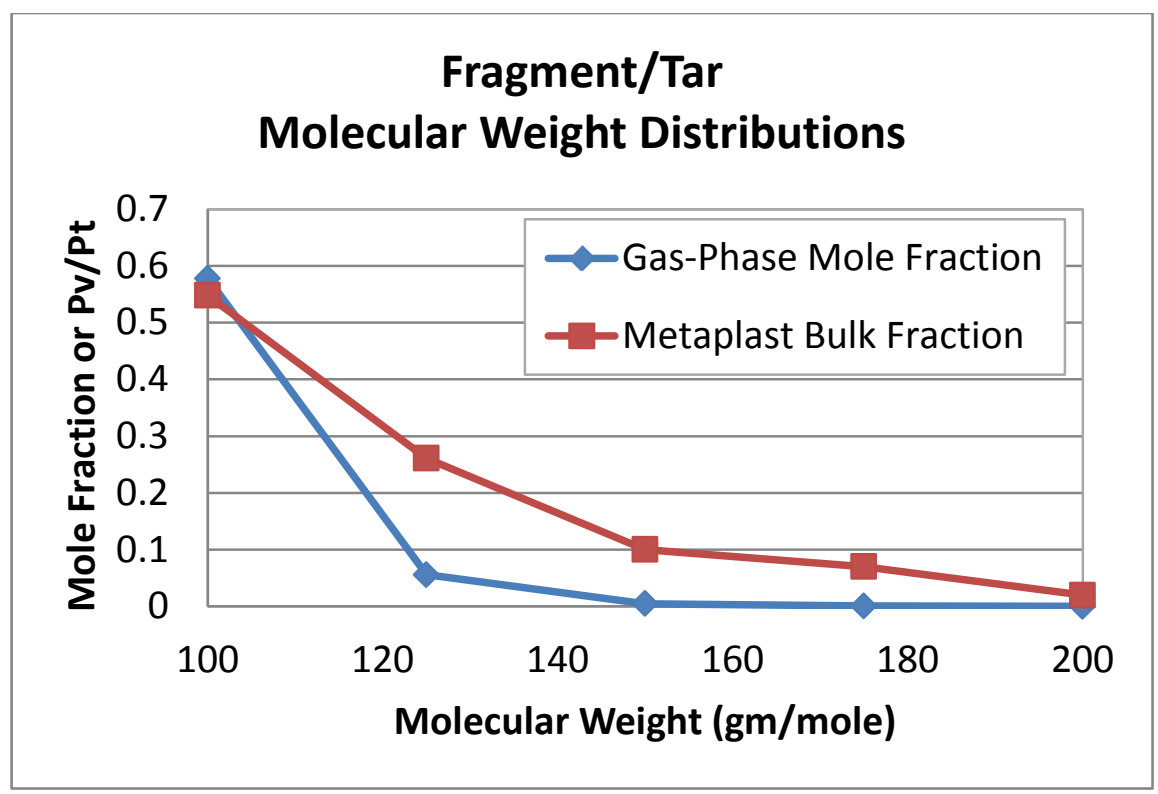

Figure 62. Distribution of equilibrium (steady-state) mole fractions of liquid tar species and their corresponding vapor species.

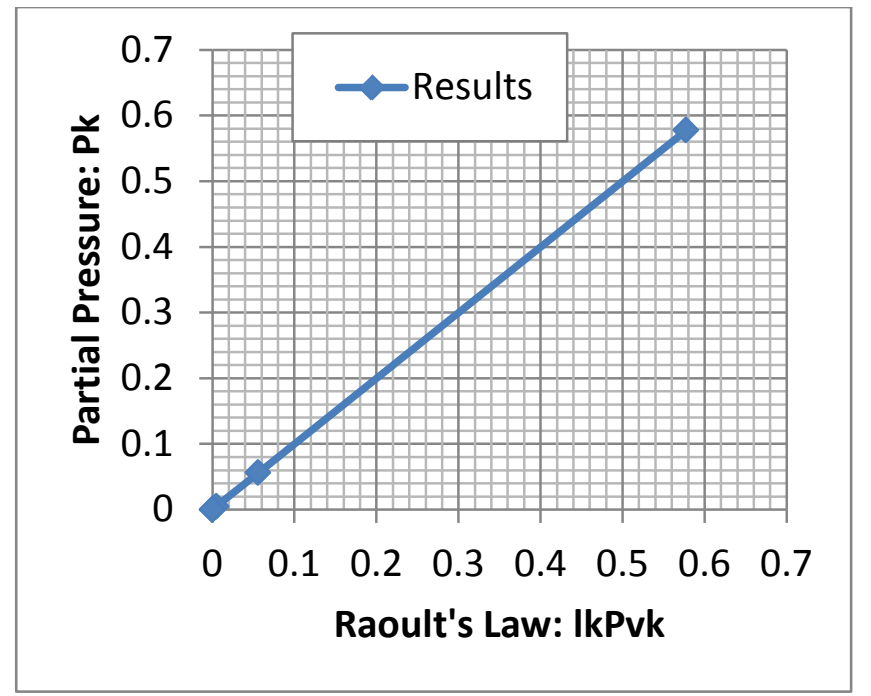

Figure 63. Comparison of partial pressure versus liquid-phase composition of a multicomponent metaplast indicates that Raoult's law for multiple component mixture is satisfied.

\subsubsection{Fragmentation Reaction}

The fragmentation reaction describes the formation of tar species in a metaplast due to thermal decomposition of the coal lattice. The fragmentation reaction is also used to identify fragment bulk species and their corresponding gaseous tar species. The stoichiometric coefficient of the reactant (a "core" bulk species) indicates the number of sites/cores in the product (a fragment bulk species). Since the real coal lattice is modeled as a Bethe Pseudo lattice, the fragmentation reaction rate is computed according to the percolation theory [33]. 
Mole fraction distributions of fragments with different numbers of sites (fragment classes) based on the percolation lattice theory are shown in Figure 64 for $1>p \geq p^{*}$ ( $p$ is the intact bridge mole fraction and $p^{*}=1 / \sigma$ is the percolation threshold). The mole fraction of each fragment class increases rapidly as the intact bridge fraction drops towards the threshold $p^{*}$. The smallest fragment (class 1 fragment) comprises the majority of the fragment population; larger fragment species do not have a significant presence until the intact bridge fraction approaches the threshold. According to Figure 64, there is not much advantage to including fragment species with molecular weights larger than $800 \mathrm{~g} / \mathrm{mole}$.

To verify the fragmentation reaction, a minimal set of reactions was set up to describe the thermal decomposition of a coal lattice, as shown in Figure 65. The coal in this validation test is $100 \%$ carbon and consists of 8 bulk species to represent core/site, bridge, char link, broken bridge/side chain, and 4 classes of lattice fragments. The coal lattice has a coordination number of 5 , which is about the highest value of all coals that have been analyzed. It is also interesting to understand the effect of the presence of the char link species CLink(B) on the decomposition of the coal lattice. Therefore, in addition to the case with all bridges breakable initially (i.e., $\left.\operatorname{LBrdg}(B)_{i}=1.0\right)$, a second simulation was carried out with permanent char links making up $20 \%$ of the connections (that is, $\operatorname{LBrdg}(\mathrm{B})_{i}=0.8$ and $\operatorname{CLink}(\mathrm{B})_{i}=0.2$ ).

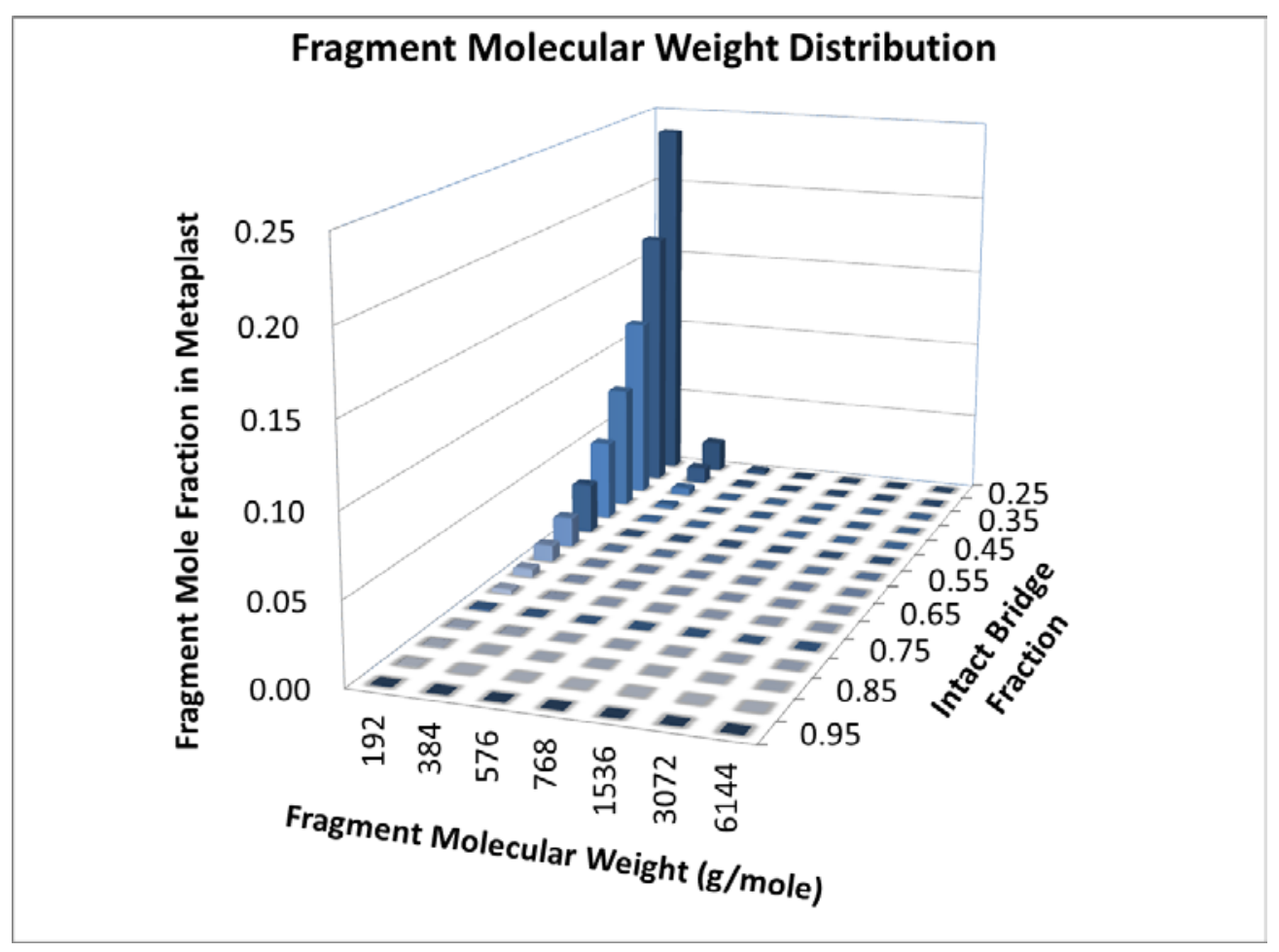

Figure 64. Fragment molecular weight (class) distribution determined by the lattice theory for intact bridge fractions greater than the percolation threshold $p^{*}$. 


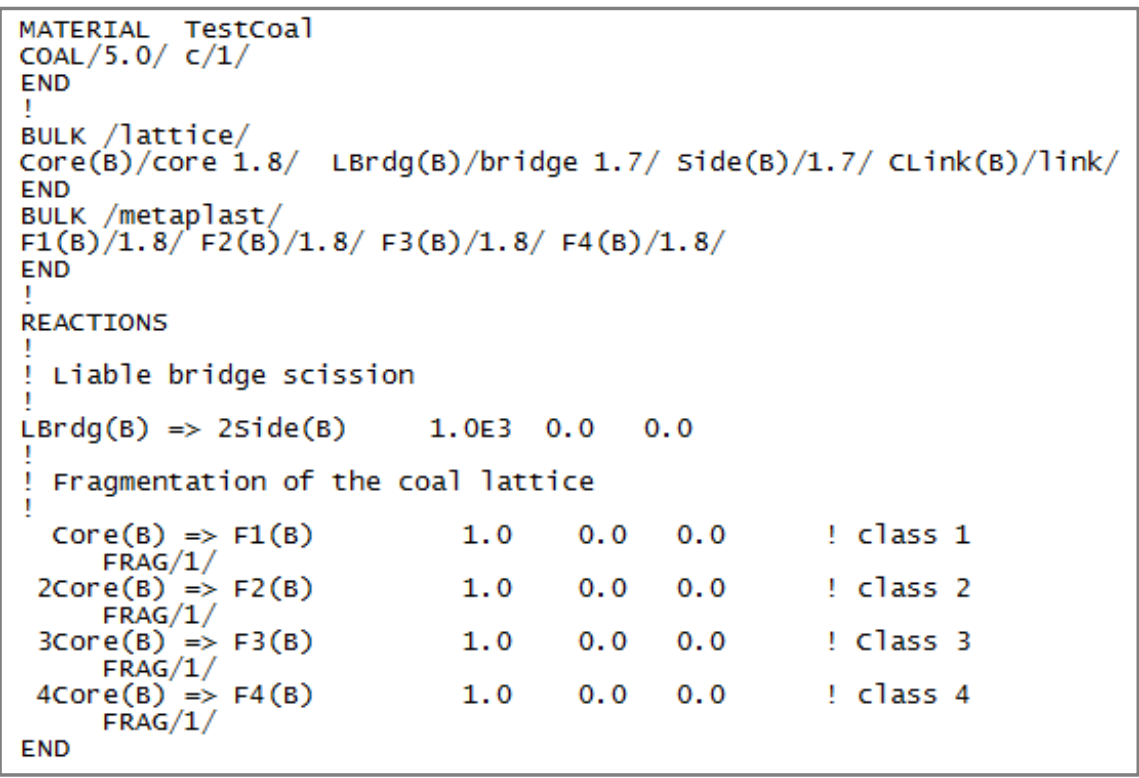

Figure 65. Simple coal thermal decomposition mechanism used to validate the fragmentation reaction.

Figure 66 to Figure 69 compare profiles of bridge species, class- 1 fragment, class- 4 fragment, and core species (equivalent to large fragments) with and without the presence of permanent char links in the coal lattice. Intuitively, permanent char links should improve the integrity of the coal lattice by holding the cores together. The effect of a strong lattice should manifest itself by promoting the formation of large fragments, or, equivalently, by reducing the level of small fragments.

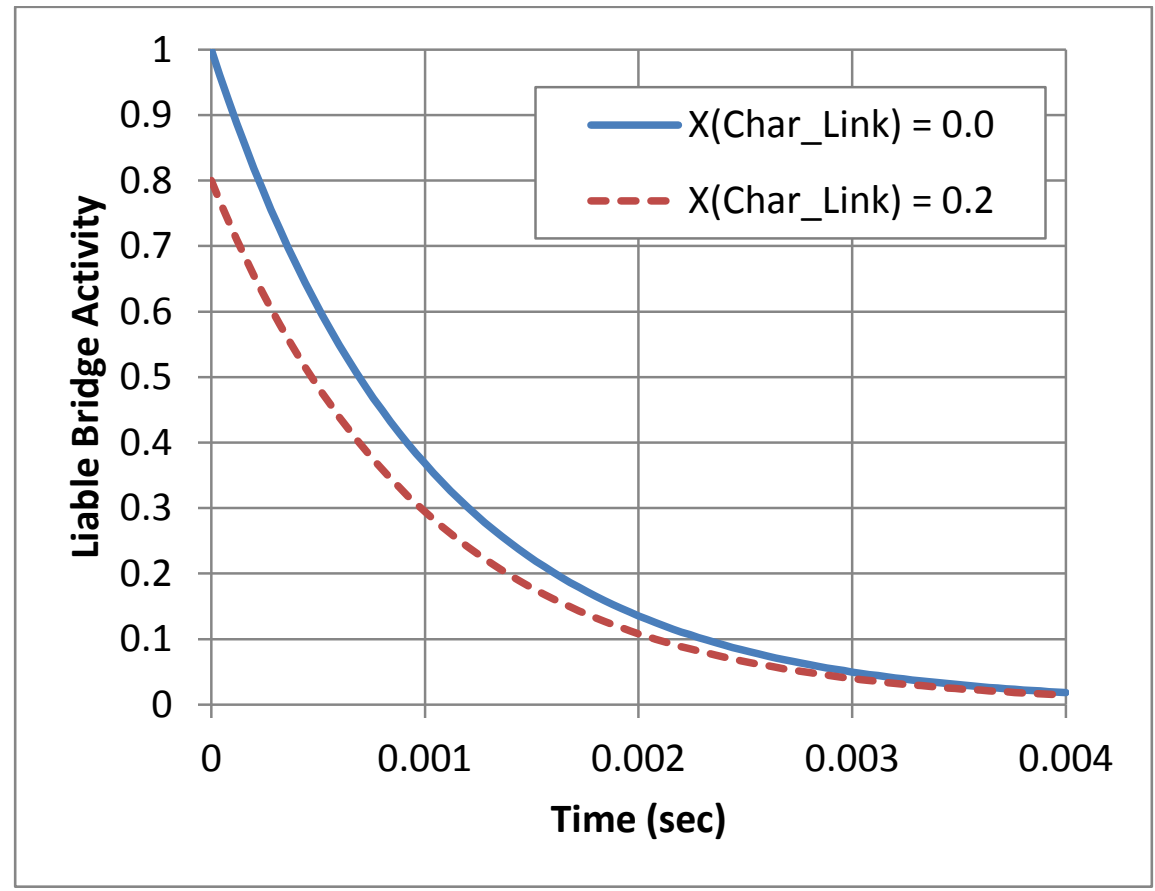

Figure 66. Effect of initial char link fraction $\mathrm{CLink}(\mathrm{B})_{i}$ on the time evolution of the liable bridge species in a pseudo Bethe lattice. 


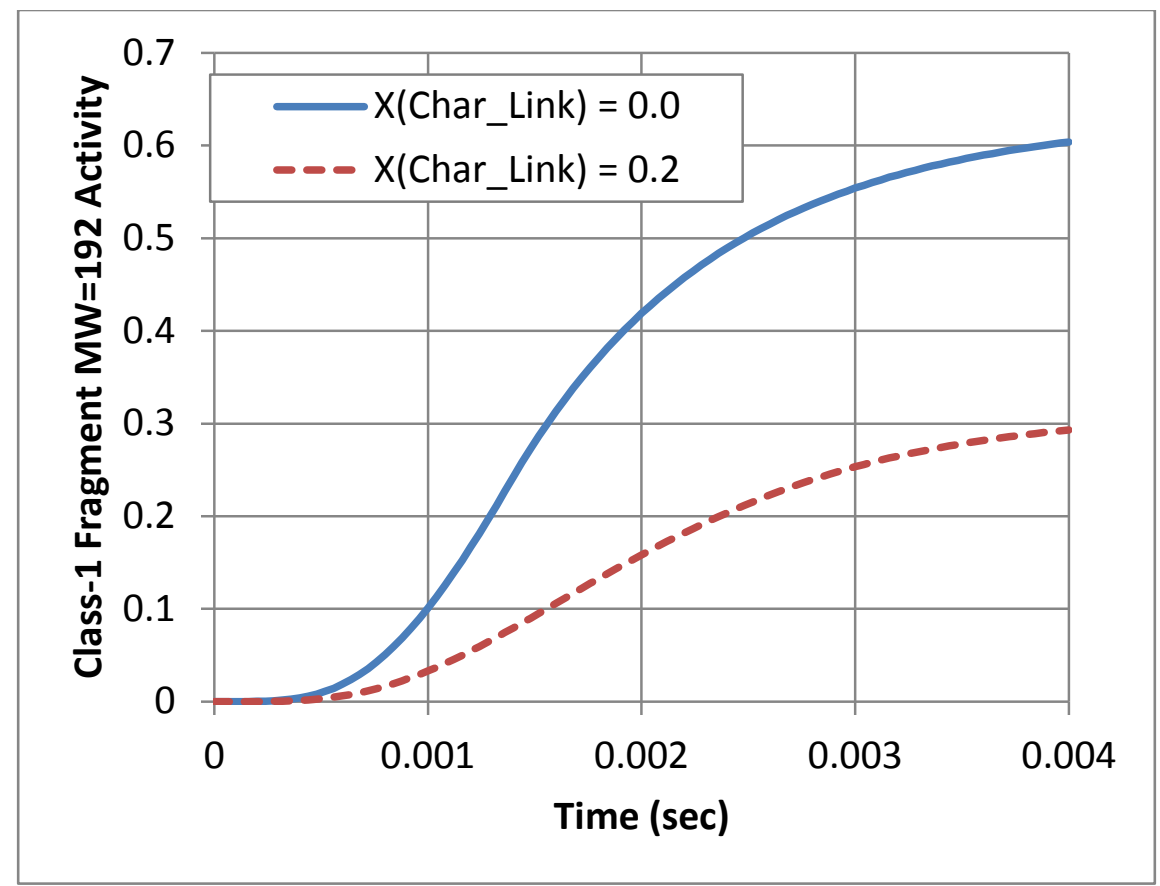

Figure 67. Effect of initial char link fraction CLink $(B)_{i}$ on the time evolution of the smallest (Class-1) fragment species.

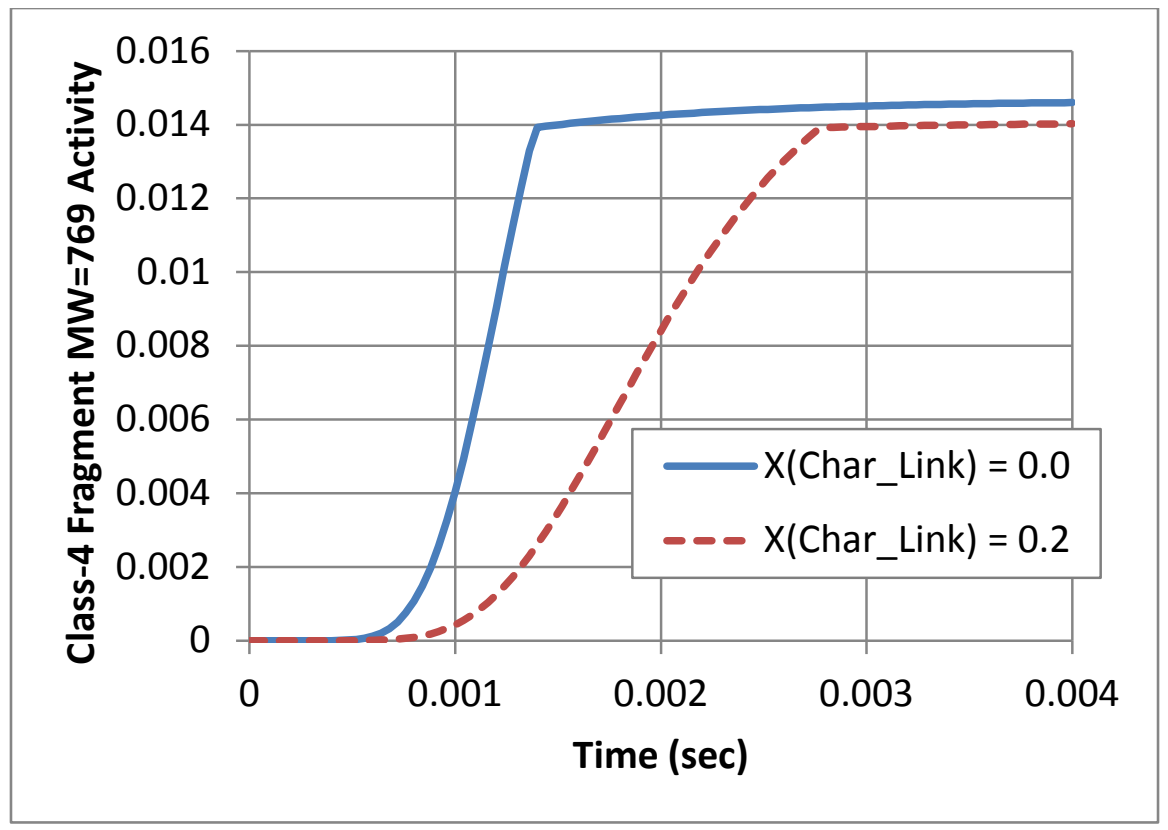

Figure 68. Effect of initial char link fraction CLink $(B){ }_{i}$ on the time evolution of a large (Class-4) fragment species. 


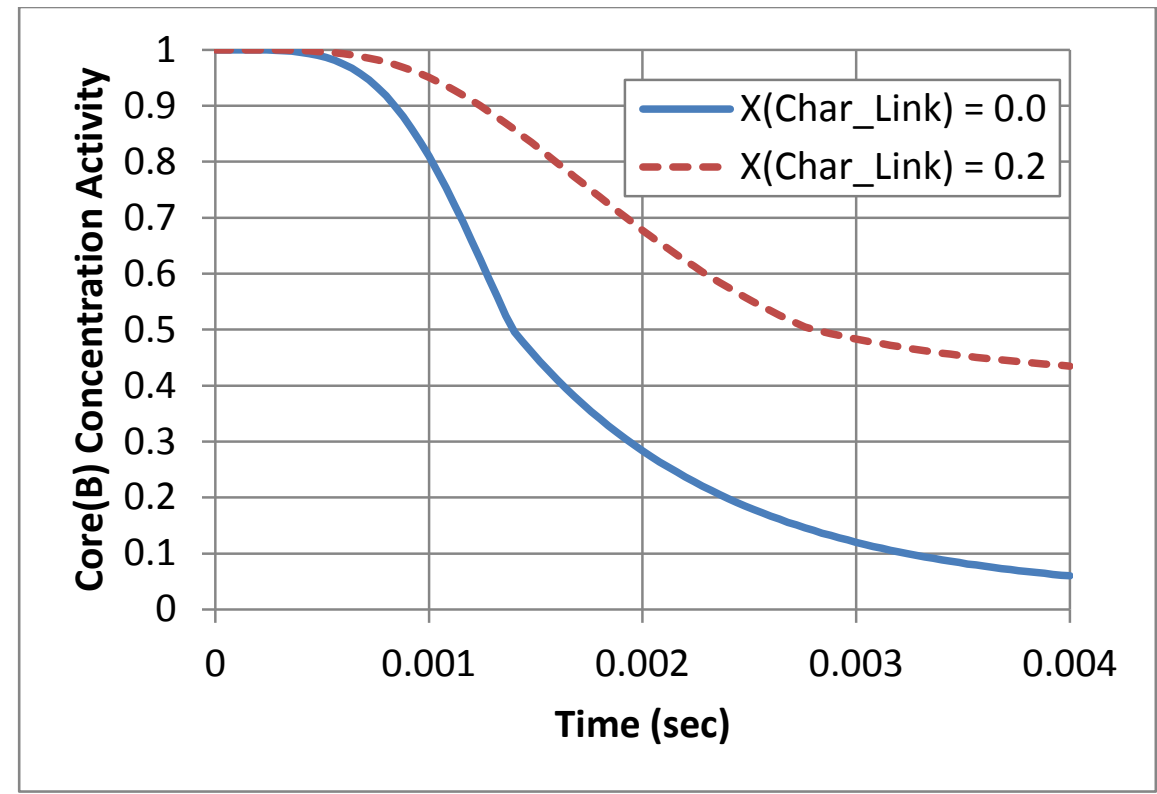

Figure 69. Effect of initial char link fraction CLink $(B)_{i}$ on the time evolution of a core species (or, equivalently, a very large finite-size fragment).

The bridge-species profile indicates, as expected, that no bridge remains intact at the end of the thermal cracking process. On the other hand, Figure 69 shows a small amount of core species left at the end for the $\operatorname{LBrdg}(\mathrm{B})_{i}=1.0$ case, while all core species in the coal lattice should have broken apart and been converted into one of the fragment species. An explanation is that the remaining core species collectively represents a small amount of large, finite-size fragments, for example, hundreds of class-1019 and tens of class- $10^{20}$ fragments in the metaplast. Because the coal chemistry model only tracks fragments containing up to 4 cores (class- 4 fragments), finite-size fragments with more than 4 cores will be categorized as part of the coal lattice and kept as Core(B).

The bridge-species profiles in Figure 66 indicate that the bridge scission rate is not affected significantly by the increase of char links in the coal lattice. On the other hand, the class-1 fragment concentration profiles in Figure 67 exhibit strong char-link influence. The presence of char links greatly reduces the production rate and the total number of the smallest class- 1 fragments, as one would expect from a strongly connected lattice. For the slightly bigger class- 4 fragment, the influence of char links on its population is not as prominent as it is on the delay of formation, as shown by Figure 68. The char-link effect on the largest fragments and on the lattice itself can be derived from the core species profiles. Figure 69 shows that the size of the coal lattice and/or the populations of large fragments are a lot higher with the presence of char links than without any char link, which is consistent with a stronger coal lattice.

\subsubsection{Initial Surface Mechanism for Coal Devolatilization}

Most kinetic parameters in a coal devolatilization mechanism, especially those for bridge breakup and light-gas release, should be common among coal ranks [33,37]. However, molecular weights of aromaticcore and bridge structures, as well as the composition of functional group species, can vary significantly from one coal to another. Consequently, a coal devolatilization mechanism is coal-specific. We focused at first on Pittsburgh \#8. The conversion of elemental and structural information of a coal into actual species is extremely complicated. Therefore, our first surface mechanism for coal was built for a wellcharacterized coal so that we could minimize uncertainties in the data and concentrate on developing the algorithm for determining the surface species that define each coal. 
In general, experimental data required to derive average species composition in a coal are:

1. Elemental composition.

2. Mass fraction of volatile matter.

3. Coordination number of the coal matrix.

4. Average molecular weight of the aromatic core.

5. Average molecular weight of the macro-structure cluster in the coal.

6. Initial intact bridge fraction.

7. Data for the light-gas released from the coal, as shown by Figure 70 [57].

The algorithm developed serves as the foundation for creating an automatic generator for coal mechanisms. The coal mechanism generator will estimate all necessary information from the elemental composition of a coal, by using empirical formulas or a coal library. The coal mechanism generator will then combine these coal-dependent species definitions with a set of surface reactions that are common among all coal types, and generate a coal surface mechanism for simulating devolatilization of the given coal.

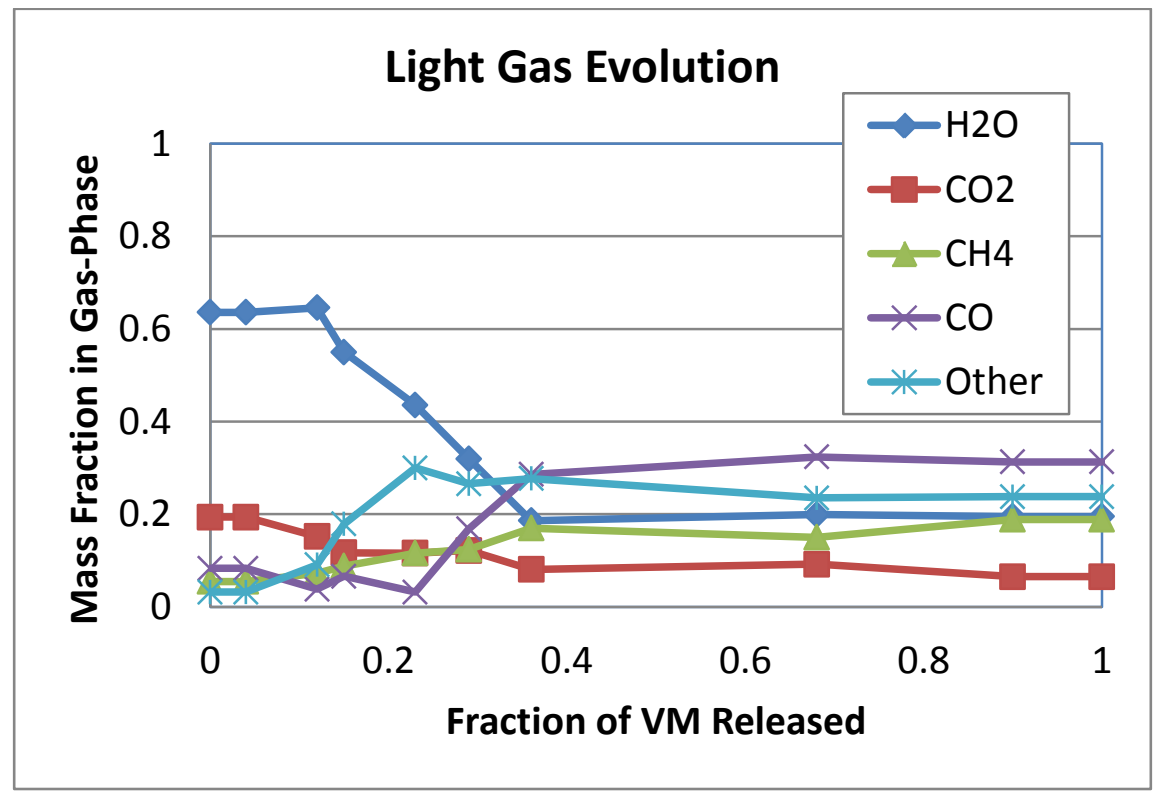

Figure 70. Measured light gas compositions as a function of volatile matter released during devolatilization (Pittsburgh \#8 coal) [57]. 


\section{Conclusions and Future Work}

The goal of this project was to enable an advanced form of reduced-order modeling for representation of key unit operations in flowsheet simulations. Using high-fidelity fluid-dynamics models as input, we extended Reaction Design's ENERGICO software, which was designed to automatically extract equivalent reactor networks (ERNs) from a CFD solution. While this technology was previously established for gas-turbine combustors, one aim of this project was to extend the methodology to gasifiers. Another key objective was to encapsulate the resulting ERN models as CAPE-OPEN UOcompliant objects that can be used seamlessly within a general-purpose, process flowsheet simulation software. The third and final objective was to explore the detailed kinetics that could be included in the ERN to provide more predictive capability for the coal gasifier representation. The reactor network extraction and CAPE-OPEN encapsulation formed the basis for developing accurate and computationally efficient reduced-order-models or ROMs for gasification/combustor processes. By adopting the CAPEOPEN architecture, such a representation of a combustor or gasifier can be combined with other unit operations to allow a plant or system simulation, providing a computationally efficient and accurate alternative to using fluid-dynamics models directly in this context. By further investing some attention to the reaction kinetics, the ERNs were shown to be more than just reduced-order models in the sense that they can predict much more information than the original CFD simulations from which they were derived.

We have achieved each of these objectives and demonstrated the full simulation workflow, from the importing of a CFD solution file to the execution of an extracted ERN reduced-order model in the process flowsheet simulator for two different coal gasifier models. The ENERGICO ERN-extraction technology was extended to allow the use of discrete-phase-material source terms and particulate information from ANSYS FLUENT CFD models in the ERN definition. A CAPE-OPEN compliant interface was implemented to encapsulate a resulting ERN model, such that it can be run within any CO-compliant process flowsheet simulation. That compliance was demonstrated by testing in both the COFE and Aspen Plus simulators.

The ability of the ERN to include more kinetics detail than the original CFD model was also demonstrated, with the prediction of $\mathrm{NO}_{x}$ and $\mathrm{SO}_{x}$ emissions as well as the prediction of polycyclic aromatic hydrocarbons (PAHs). That concept was further established by the design and development of an advanced Coal Kinetics Module that allows a much more physically based description of the coal devolatilization process than typically used today in CFD models, which rely on empirically derived devolatilization rates.

The validation studies performed during this project focused primarily on software verification, software-integration testing, and the validation of the ERN as an effective ROM for representing the original CFD model. In future work, we plan to engage more closely with industrial gasifier manufacturers and with NETL as possible, in order to validate and extend the kinetics models that form the core of the ERN ROM. Accurate predictions of emissions and conversion efficiencies are key to improving coal gasifier/combustor designs. High-fidelity predictions are also important for enabling effective use of these models in plant and system-level designs, or in operational planning. Finally, the ability to predict trace species in the system and to map that information back onto the geometry, as demonstrated in this work, provides insights into the location of certain processes and by-product concentrations that may be important for sensor placement or reactor down-time mitigation. 


\section{Acronyms}

\begin{tabular}{|c|c|}
\hline ANN & Artificial Neural Network. \\
\hline APECS & Advanced Process Engineering Co-Simulator project at NETL. \\
\hline CAPE & Computer-Aided Process Engineering \\
\hline CFD & Computational Fluid Dynamics. \\
\hline CGNS & CFD General Notation System for CFD analysis data. \\
\hline CKCAPE & file format for exporting ERN from CHEMKIN-PRO to CO interface. \\
\hline $\mathrm{CO}$ & CAPE-OPEN. \\
\hline $\mathrm{COCO}$ & A CAPE-OPEN compliant simulator. \\
\hline COFE & CAPE-OPEN Flowsheet Environment. \\
\hline Co-LaN & CAPE-OPEN Laboratories Network. \\
\hline $\mathrm{COM}$ & Microsoft's Component Object Model. \\
\hline CORBA & Common Object Request Broker Architecture. \\
\hline CPD & Chemical Percolation Devolatilization model. \\
\hline DAE & Distributed Activation Energy reaction type. \\
\hline DLL & Dynamic Link Library, Microsoft's implementation of shared libraries. \\
\hline DPM & Discrete-Phase Material. \\
\hline DVC & Devolatilization-Vaporization-Crosslinking model. \\
\hline ERN & Equivalent Reactor Network. \\
\hline FG & Functional Group model. \\
\hline GUI & Graphical User Interface. \\
\hline IGCC & Integrated Gasifier/Gas-Turbine Combined-Cycle Systems. \\
\hline MWD & Molecular Weight Distribution. \\
\hline NETL & National Energy Technology Laboratory. \\
\hline PFR & Plug-Flow Reactor model in CHEMKIN. \\
\hline PSR & Perfectly-Stirred Reactor model in CHEMKIN. \\
\hline $\mathrm{ROM}$ & Reduced Order Model. \\
\hline SPAMM & Simultaneous Particle and Molecule Modeling method. \\
\hline $\mathrm{UO}$ & Unit Operations. \\
\hline UTRC & United Technologies Corporation. \\
\hline VB6 & Visual Basic version 6 (1998). \\
\hline
\end{tabular}




\section{References}

1. Aspen Plus, Cambridge, MA: AspenTech, Inc., 2009.

2. ENERGICO Release 30112: Reaction Design, 2012.

3. S. E. Zitney, M. Osawe, L. Collins, E. Ferguson, D. Sloan, W. Fiveland, and J. Madsen, "Advanced Process Co-Simulation of the FutureGen Power Plant," The 31st International Technical Conference on Coal Uitilization \& Fuel Systems, Clearwater, Florida, 2006.

4. S. A. Drennan, C.-P. Chou, A. F. Shelburn, D. W. Hodgson, C. Wang, C. V. Naik, E. Meeks, and H. Karim, "Flowfield-Derived Equivalent Reactor Networks for Accurate Chemistry Simulation in Gas-Turbine Combustors," Proceedings of ASME Turbo Expo, GT2009-59861, 2009.

5. "CO-Lan ( The CAPE [Computer Aided Process Engineering] Laboratories Network)," http://www.colan.org/,

6. D. W. Kendrick, A. Bhargava, M. B. Colket, W. A. Sowa, D. J. Maloney, and K. H. Casleton, "NO Scaling Characteristics for Industrial Gas Turbine Fuel Injectors," ASME Turbo Expo, 2000.

7. I. V. Novosselov, P. C. Malte, S. Yuan, R. Srinivasan, and J. C. Y. Lee, "CHEMICAL REACTOR NETWORK APPLICATION TO EMISSIONS PREDICTION FOR INDUSTIAL DLE GAS TURBINE," Proceedings of GT2006, 2006-90282, 2006.

8. C. Russo, G. Mori, V. V. Anisimov, and J. Parente, "Micro Gas Turbine Combustor Emissions Evaluation Using the Chemical Reactor Modelling Approach," Proceedings of ASME Turbo Expo, GT2007-27687, 2007.

9. D. Benedetto, S. Pasini, M. Malcitelli, C. La Marca, and L. Tognotti, "NOX Emission Prediction from 3D Complete Modelling to Reactor Network Analysis," Combustion Science and Technology, 153: 279294, 2000.

10. M. Falcitelli, S. Pasini, N. Rossi, and L. Tognotti, "CFD+reactor network analysis: an integrated methodology for the modeling and optimization of industrial systems for energy saving and pollution reduction ." Applied Thermal Engineering, 22 971-979, 2002.

11. T. Faravelli, L. Bua, A. Frassoldati, A. Antifora, L. Tognotti, and E. Ranzi, "A New Procedure for Predicting $\mathrm{NO}_{x}$ Emissions from Furnaces," Computers and Chemical Engineering, 25: 613-618, 2001.

12. G. Liu and S. Niksa, AFRC International Symposium, 2003.

13. F. Bezzo, S. Macchietto, and C. C. Pantelides, "General Hybrid Multizonal/CFD Approach for Bioreactor Modeling," AIChE J., 49: 2133-2148, 2003.

14. CHEMKIN-PRO Release 15112, San Diego, CA: Reaction Design, 2012.

15. "The Model Fuels Consortium," $\leq$ http://www.modelfuelsconsortium.com $>P R$ Newswire, United Business Media, October 17, 2005.

16. E. Meeks, H. Ando, C.-P. Chou, A. M. Dean, D. Hodgson, M. Koshi, I. Lengyel, U. Maas, C. V. Naik, K. V. Puduppakkam, R. Reitz, C. Wang, and C. K. Westbrook, "New Modeling Approaches Using Detailed Kinetics for Advanced Engines," in The Seventh International Conference on Modeling and Diagnostics for Advanced Engine Systems (COMODIA 2008). Sapporo, Japan, 2008.

17. C. V. Naik and A. M. Dean, "Modeling High Pressure Ethane Oxidation and Pyrolysis," Proceedings of the Combustion Institute, 32, 2008.

18. K. Puduppakkam, L. Liang, C. V. Naik, E. Meeks, and B. G. Bunting, "Combustion and Emissions Modeling of an HCCI Engine Using Model Fuels," SAE Technical Paper Series, SAE2009-01-0669, 2009.

19. P. Dagaut, P. Glarborg, and M. U. Alzueta, "The Oxidation of Hydrogen Cyanide and Related Chemistry," Progress in Energy and Combustion Science, 34: 1-46, 2008.

20. C. L. Rasmussen, A. E. Rasmussen, and P. Glarborg, "Sensitizing Effects of NOx on CH4 Oxidation at High Pressure," Combustion and Flame, 154: 529-545, 2008. 
21. M. J. Bockelie, M. K. Denison, Z. Chen, T. Linjewile, C. L. Senior, and A. F. Sarofim, "CFD Modeling for Entrained Flow Gasifiers in Vision 21 Systems," Proceedings of the 19th Annual International Pittsburgh Coal Conference, 2002.

22. R. E. Mitchell, "An Intrinsic Kinetics-based Particle-Population Balance Model for Char Coxidation During Pulverized Coal Combustion," Proceedings of the Combustion Institute, 28: 2261-2270, 2000.

23. R. H. Hurt and R. E. Mitchell, "Unified High-Temperature Char Combustion Kinetics for a Suite of Coals of Various Rank," Proceedings of the Combustion Institute, 24: 1243-1250, 1992.

24. FLUENT, Lebanon, NH: Ansys, Inc., 2009.

25. C. V. Naik, E. Meeks, and S. Drennan, "Detailed Chemical Kinetic Mechanism for Methane and Ethane Combustion including NOx Emissions for Gas Turbines Applications," in Fall Technical Meeting of the Western States Section of the Combustion Institute. Irvine, CA, 2009.

26. J. A. Miller and C. T. Bowman, "Mechanism and Modeling of Nitrogen Chemistry in Combustion," Prog. Energy Combust. Sci., 15: 287-338, 1989.

27. "COCO CAPE-OPEN to CAPE-OPEN Simulation Environment," http://www.cocosimulator.org/, April 2010.

28. D. B. Anthony, J. B. Howard, H. C. Hottel, and H. P. Meissner, Proceedings of the Combustion Institute, 15: 1303-1317, 1975.

29. P. R. Solomon and D. G. Hamblen, in Chemistry of Coal Conversion, R. H. Schlosberg, Ed.: Plenum Press, 1985, pp. 121-251.

30. D.M. Grant, R. J. Pugmire, T. H. Fletcher, and A. R. Kerstein, Energy and Fuels, 3: 175-186, 1989.

31. Y. Zhao, M. A. Serio, R. Bassilakis, and P. R. Solomon, "A Method of Predicting Coal Devolatilization Behavior Based on the Elemental Composition," Proceedings of the Combustion Institute, 25: 553560, 1994.

32. D. Genetti, T. H. Fletcher, and R. J. Pugmire, "Development and Application of a Correlation of 13C NMR Chemical Structural Analyses of Coal Based on Elemental Composition and Volatile matter Content," Energy \& Fuels, 13: 60-68, 1999.

33. D. M. Grant, R. J. Pugmire, T. H. Fletcher, and A. R. Kerstein, "Chemical Model of Coal Devolatilization Using Percolation Lattice Statistics," Energy \& Fuels, 3: 175-186, 1989.

34. P. R. Solomon, D. G. Hamblen, R. M. Carangelo, M. A. Serio, and G. V. Deshpande, "General Model of Coal Devolatilization," Energy \& Fuels, 2: 405-422, 1988.

35. S. Niksa, and A. R. Kerstein, "FLASHCHAIN Theory for Rapid Coal Devolatilization Kinetics. 1. Formulation," Energy \& Fuels, 5: 647-665, 1991.

36. S. Niksa, "FLASHCHAIN Theory for Rapid Coal Devolatilization Kinetics. 3. Modeling the Behavior of Various Coals," Energy \& Fuels, 5: 673-683, 1991.

37. P. R. Solomon, and M. B. Colket, "Coal Devolatilization," Proceedings of the Combustion Institute, 17: 131-143, 1979.

38. P. R. Solomon, D. G. Hamblen, R. M. Carangelo, and J. L. Krause, "Coal Thermal Decomposition in an Entrained Flow Reactor: Experiments and Theory," Proceedings of the Combustion Institute, 19: $1139-1149,1982$.

39. M. A. Serio, D. G. Hamblen, J. R. Markham, and P. R. Solomon, "Kinetics of Volatile Product Evolution in Coal Pyrolysis: Experiments and Theory," Energy \& Fuels, 1: 138-152, 1987.

40. P. R. Solomon, and D. G. Hamblen, "Pyrolysis," in Chemistry of Coal Conversion, R. H. Schlosberg, Ed. New York: Plenum Press, 1985, pp. 121-251.

41. P. E. Unger, and E. M. Suuberg, "Internal and External Mass Transfer Limitations in Coal Pyrolysis," ACS Div. of Fuel Chemistry preprints, 28: 278-291, 1983.

42. C. J. Pope, and J. B. Howard, "Simultaneous Particle and Molecule Modeling (SPAMM): An Approach for Combining Sectional Aerosol Equations and Elementary Gas-Phase Reactions," Aerosol Science and Technology, 27: 73-94, 1997.

43. R. S. Jupudi, V. Zamansky, and T. H. Fletcher "Prediction of Light Gas Composition in Coal Devolatilization," Energy \& Fuels, 23: 3063-3067, 2009.

44. M. S. Solum, R. J. Pugmire, and D. M. Grant, "Carbon-13 Solid-State NMR of Argonne-Premium Coals," Energy \& Fuels, 3: 187-193, 1989. 
45. Leeds University, "Sulphur Mechanism Extension to the Leeds Methane Mechanism," http://www.chem.leeds.ac.uk/combustion/mechanisms/leedssox52.dat,

46. W. Nimmo, E. Hampartsoumain, K.J. Hughes, and A.S. Tomlin, "Experimental and Kinetics Studies on the Effect of Sulfur-Nitrogen Interactions on NO Formation in Flames," Proceedings of The Combustion Institute, 27: 1419-1426, 1998.

47. K. J. Hughes, M.A. Blitz, M.J. Pilling, and S.H. Robertson, "A Master Euqation Model for the Determination of Rate Coefficients in the $\mathrm{H}+\mathrm{SO} 2$ System," Proceedings of The Combustion Institute, 29: 2431-2437, 2002.

48. M. U. Alzueta, R. Bilbao, and P. Glarborg, "Inhibition and Sensitization of Fuel Oxidation by SO2," Combust. and Flame, 127: 2234-2251, 2001.

49. M. Binoist, B. Labegorre, F. Monnet, P.D. Clark, N.I. Dowling, M. Huang, D. Archambault, E. Plasari, and P.-M. Marquaire, "Kinetic Study of the Pyrolysis of H2S," Ind. Eng. Chem. Res., 42: 3943-3951, 2003.

50. S. Niksa, J.J. Helble, and N. Fujiwara, "Kinetic Modeling of Homogeneous Mercury Oxidation: the Impoprtance of $\mathrm{NO}$ and $\mathrm{H} 2 \mathrm{O}$ in Predicting Oxidation in Coal-Derived Systems," Environmental Science \& Technology, 35: 3701-3706, 2001.

51. N. C. Widmer, J. West, and J.A. Cole, "Thermochemical Study of Mercury Oxidation in Utility Boiler Flie Gases," Proceedings of the Air \& Waste Management Association's 93rd Annual Conference, Salt Lake City, Utah, 2000.

52. J. F. Roesler, R.A. Yetter, and F.L. Dryer, "Kinetic Interactions of $\mathrm{CO}, \mathrm{NOx}$, and $\mathrm{HCl}$ Emissions in Postcombustion Gases," Combust. and Flame, 100: 495-504, 1995.

53. J. R. Edwards, R.K. Srivastava, and J.D. Kilgroe, "A Study if Gas-Phase Mercury Speciation Using Detailed Chemical Kinetics," Journal of the Air \& Waste Management Association, 51: 869-877, 2001.

54. M. Xu, Y. Qiao, C. Zheng, L. Li, and J. Liu, "Modeling of Homogeneous Mercury Speciation Using Detailed Chemical Kinetics," Combust. and Flame, 132: 208-218, 2003.

55. M. Frenklach, and Wang, H.,, "Detailed Mechanism and Modeling of Soot Particle Formation," in $\underline{\text { Soot }}$ Formation in Combustion: Mechanisms and Models, H. Bockhorn, Ed. Berlin: Springer-Verlag, 1994, pp. 165-192.

56. D. B. Anthony, J. B. Howard, H. C. Hottel, and H. P. Meissner, "Rapid Devolatilization of Pulverized Coal," Proceedings of the Combustion Institute, 15: 1303-1317, 1975.

57. D. B. Genetti, "An Advanced Model of Coal Devolatilization Based on Chemical Structure," in Department of Chemical Engineering, Master of Science: Brigham Young University, 1999. 